\title{
III. Formulating Models for Structured Populations
}

\author{
J.A.J. Metz \& O. Diekmann
}

\section{Introduction: six examples for later use}

By now the examples from chapter I and/or the subsequent theoretical elaboration in chapter II should have given you a taste for structured population models. In this chapter we shall develop a do-it-yourself kit enabling you to build models incorporating various amounts of biological detail.

We start in this section by introducing six examples that will be used throughout the chapter to illustrate various complications of the modelling process. In the next section we shall expose some of our modelling philosophy and in Section 3 the mathematical formalism for dealing with "mass" balance at the population level will be explicated in detail. Section 4 deals with some alternative parametrizations of the individual and population state spaces and sections 5 and 6 treat various kinds of limit arguments which may be used to simplify the model formulation. Finally appendix A contains a short refresher of vector notation and calculus in $\mathbb{R}^{n}$ written to assist readers with a mainly biological background, appendix $B$ considers the extension of the formalism necessary to deal with stochastic components in the continuous $i$-state movement, a subject otherwise outside the scope of these notes, and appendix $C$ gives the complete $p$-equations of the six examples introduced in this section (and with that the answers to most of the exercises in this chapter).

\section{EXAMPLE 1.1. The invertebrate functional response continued}

This example has already been introduced in I.2. There we made the simplifying assumption that pursuit and eating durations could safely be neglected. If we wish to take account of these durations we need a more complicated $i$-state space than the one spanned by satiation alone. Here we shall give a verbal account of how such a state space may be constructed. Later in this chapter we shall derive the corresponding p-equations. A more detailed discussion of the biological rationale for our assumptions as well as various extensions may be found in Metz \& van Batenburg (1985a,b).

We start asssuming that for a searching predator the rate of search, $g_{0}$, depends only on its satiation, and that the rate of decrease of satiation, $-f_{0}$, depends only on that satiation itself as $f_{0}(s)=-a s$. Accordingly for a searching predator its satiation still is a sufficient state description.

When a prey is sighted our predator starts pursuing. We shall assume here that the future course of the pursuit depends only on the distance which still remains between predator and prey. Moreover we assume that 1) this distance shortens at a constant speed, 2) prey escape, by flying away, at a constant rate $\mu$, and 3) the distance at which pursuit is started depends only on the predator's satiation at that time (remember: the width of the search field depended on satiation). Accordingly for a pursuing predator the pair satiation cum distance-to-prey suffices as a state description.

When the predator arrives within a sufficient distance of its prey it strikes. We shall assume that the probability that this strike is successful is a constant $q$. A successful strike ends with a transition to eating. Otherwise the prey escapes and the predator starts searching again.

For the sake of the exposition we shall assume here that our predator eats at a constant speed, that a prey item is eaten in toto and that prey size also is constant. Accordingly remaining meal size and satiation together suffice as a state description for an eating predator.

To complete our model description we still have to prescribe the dynamics of the satiation during pursuit and eating. For pursuing predators we shall assume that satiation decreases at the same rate as during search. For eating predators we shall assume that satiation rises as $f_{1}=f_{0}+u$, where $u$ denotes the rate of ingestion.

A point in favour of the state space described above is that the state variables all have an immediate physical interpretation allowing a direct simultaneous measurement, at least in principle. Such a type of model formulation should generally be preferred as it makes it relatively easy to extend the model to different experimental circumstances. However, the state space itself has a rather odd appearance. It consists of three unconnected pieces, the predator jumping from one piece to the other in the course of the predation process. In general this is exactly how it is. However, the 
simplifying assumptions made above were made with the specific purpose in mind of arriving at a state space allowing an easier visualisation. For, using these assumptions, we can transform the dimensions of both additional state variables to time by dividing the first one by the speed of pursuit after subtracting the strike distance and by dividing the second one by the speed of eating. The resulting two state variables then can be combined by simple addition into one new state variable: maximum time still to be spent handling a prey. This new state variable we shall call $\tau$. For searching predators we shall put $\tau=0$. The predator's $i$-state space then is contained in the product of the $s$ - and $\tau$ axes.

For practical calculations it is important that we delimit ourselves to the reachable states only. The resulting $i$-state space $\Omega$ for Holling's mantid is depicted in figure 1.1, together with some segments of possible trajectories representing various behaviour sequences that may occur after a prey has entered the predator's search field. Boundary segment (2) is given by $\tau=\tau_{m}(s)$,i.e. the maximum time that handling a prey sighted at satiation $s$ may take. Boundary segments (1) and (4) both satisfy $d s / d t=-a s+u$.

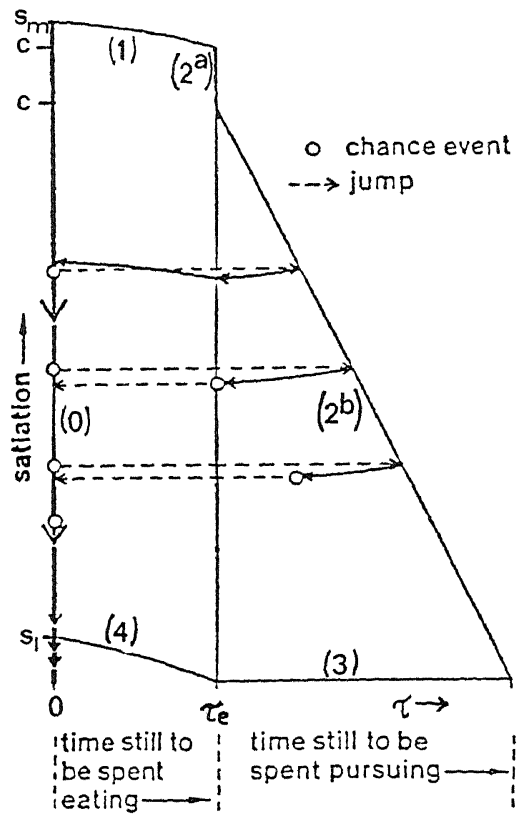

Figure 1.1: The individual state space, $\Omega$, for Holling's mantid. In the state space some segments of trajectories are depicted showing the various possible events that may happen after the sighting of a prey. The starting points of the segments on the s-axis are arbitrary. The upper segment corresponds to a successful prey capturing sequence. In the second segment the strike is unsuccessful and in the third segment the prey escapes during pursuit. The segments of the boundary of $\Omega$ are numbered for later reference. From Metz \& van Batenburg (1985a).

\section{EXAMPLE 1.2: Size dependent reproduction in ectothermic animals, continued}

This example has already been introduced in I.3. The only addition which we shall make in this chapter is that we shall relax the assumption that the death rate is constant.

Under laboratory conditions the death rate of Daphnids does not seem to depend on size except that large animals may die from starvation if feeding levels are suddenly and strongly reduced. However there is a pronounced dependence on age. The maximum age Daphnia magna may reach is about 70 days. Moreover there has sometimes been observed an increased death rate already considerably prior to this age. In Diekmann et al (1984) the following death rates have been proposed

$$
\mu(l, a)= \begin{cases}\mu_{0} & a<a_{\max } \\ \infty & a \geqslant a_{\max }\end{cases}
$$




$$
\mu(l, a)=\mu_{0}+\psi(a) \text { with } \psi(a)=\left(a_{\max }-a\right)^{-1}
$$

The reachable $i$-state space $\Omega$ for this example is depicted in figure 1.2 (see also exercise 1.3).

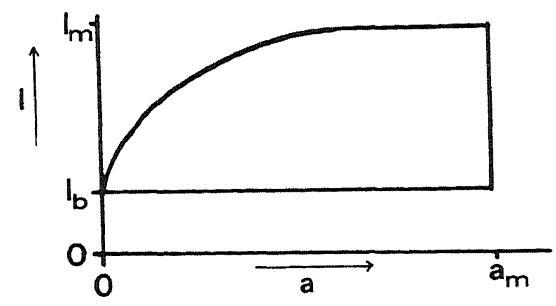

Figure 1.2. The reachable $i$-state space of the Daphnia model

EXERCISE 1.3: Prove that also for death rate (1.2) animals never reach an age higher than $a_{\max }$. What happens if we replace $\psi(a)$ by $\epsilon /\left(a_{\max }-a\right)$ and let $\epsilon$ go to zero? Hint: The answer to the last question is given at the end of section 5.2.

EXAMPLE 1.4: Reproduction by binary fission, continued

Just as in the previous example we may also have to introduce age as an additional state variable in the binary fission model from I.4. One reason may be that cells can only divide after sufficient time has elapsed to duplicate their DNA, independent of their size. The state space then again is a subset of the age size plane.

EXERCISE 1.5: Assuming that cells only can divide after they have reached a certain age $a_{0}$ construct the subset of the age size plane that is reachable by newborn cells starting life at all possible sizes $x>0$. Assume that there are no further restrictions on division age or division size and that the division rate $b(a, x) \leqslant b_{\max }<\infty$, and assume that cell growth rate $V$ depends only on cell size and that

$$
0<V_{\min } \leqslant V(x) \leqslant V_{\max }<\infty \text {. }
$$

Which region of the age size plane can be reached by their first generation descendants, their second generation descendants etc? What, therefore, does the reachable state space $\Omega$ for naturally occurring cells look like?

Hints: First assume that $V(x)=V_{0}$ to get the feel of the problem. The answers to this exercise can be found in chapter V.

\section{EXAMPLE 1.6: Colony size distribution in the diatom Asterionella.}

Asterionella is a planctonic diatom which occurs in small star-shaped colonies. These colonies generally have sizes which are powers of two. The probable mechanism behind this phenomenon is that cells within one colony divide synchronously, two daughter cells staying linked by an (inanimate) bond, thereby doubling colony size, and that colonies break into equal parts as a result of the progressive weakening of the bonds over time.

In natural populations colony size distribution varies considerably. This probably is related to the growth rate of the population: when interdivision times are short e.g. as a result of better nutritional conditions, colony size will tend to be larger. The question therefore is how colony size distribution relates to the distribution of the interdivision times and hence to population growth rate.

The previous discussion makes clear that at least the ages of the various bonds should be among the state variables characterizing a colony. (Of course we could use other quantities, like bond strength, which are monotonically related to bond age, but doing so would force us to make additional assumptions, unbacked by any data, without affecting our predictions at the population level.) For instance in a colony of size $2^{3}$ there is one oldest bond of age $a_{3}$, two bonds of age $a_{2}<a_{3}$ and four bonds of age $a_{1}<a_{2}$. To keep things as simple as possible we shall assume that cell division is also (cell) age dependent. We do not need a separate state variable representing cell age, however, as this equals the age of the youngest bond $a_{1}$, except in colonies of size $2^{0}$, i.e. single cells. Our $i$-state space therefore corresponds to a (subset of) $\mathbb{R}^{+} \cup \bigcup_{k=1}^{\infty}\left(\mathbb{R}^{+}\right)^{k}$, where $\mathbb{R}^{+}$denotes the non-negative half axis. If bonds break at exactly age $A$ and there is no lower bound to the age at division the reachable $i$-state space $\Omega$ equals

$$
\left\{a_{0} \mid A \leqslant a_{0}\right\} \cup \bigcup_{n=1}^{\infty}\left\{\left(a_{1}, . ., a_{n}\right) \mid 0 \leqslant a_{1}<\cdots<a_{n}<A\right\} .
$$

EXERCISE 1.7: What does $\Omega$ look like when bonds may break at any age but always all bonds older than a certain age 
break together? How does it change $\Omega$ if we assume that there is a minimal age $D$ before division can occur?

REMARK 1.8: To ensure convergence to a stable colony size distribution we shall have to assume some spread in the ages at which cell division occurs (see exercise 1.9 and the treatment of the Asterionella problem in IV.3.2). However, from a strict mechanistic point of view this assumption is incompatible with the assumption of perfect synchrony of division: the assumption that cell age is the sole determinant of division implies the independence of the separate cells within a colony. The explanation for this seeming inconsistency is that age is only a convenient but rather artificial state variable, which need not even be related in a one to one manner to the "real" $i$-state process. One may think for example that in reality division is contingent upon the accumulation of sufficient energy or nutrients, like in the size based model from I.4, except that the division threshold should be asssumed to be a fixed quantity to ensure the independence of mothers and daughters. If colonies are swept through regions with different light intensities or nutrient availabilities by turbulent water movement then the microscopic differences in environmental histories of the various colonies will give rise to a stochastic appearance of the $i$-state processes of representative cells of separate colonies but cells within one colony develop in exactly the same manner. (A prediction from our mechanistic picture would be that in those cases where colonies have distinct "outer" cells (as in colonies of size four) these outer cells should start dividing slightly earlier. This indeed has been observed). In the age type description we simply take account of the apparent distribution of the ages at which division occurs, without bothering about the detailed generating mechanism. (A further discussion of the strengths and weaknesses of age based models may be found in chapter IV.)

EXERCISE 1.9: Give an explicit expression for the age distribution $n(t, a)$ for a cell population in which all cells exactly on reaching age one divide into two daughters of age zero. Assume that there are no cell deaths. Call the initial age distribution $n(0, a)=n_{0}(a)$. Does $n(t, a)$ converge to a stable age distribution? Also given an expression for the rate $b(t)$ at which new cells are born into the population.

\section{Example 1.10 The prey-predator-patch (PPP) problem}

In the first three examples the individuals were indeed individuals in the biological sense. In the last example we no longer dealt with individuals in the strict sense, but the state parametrization still was in terms of individual properties. In this example we shall go still one step further: our individuals will be patches and the $i$-state variables will be population sizes.

Often prey occur in local patches, e.g. spider mite colonies on single or a few leaves (see the contribution by Sabelis in part B of this volume). Such prey patches are started by a single inseminated foundress. Pure prey patches end their existence as a result of the local exhaustion of resources and the concomitant emigration of the prey, or the arrival of a predator. Till that time the local prey population grows about exponentially to a first approximation. Within a prey patch a predator almost need not search for prey so its predation rate may to a first approximation be assumed to be constant. By the same argument a local predator population will grow approximately exponentially till either all prey are consumed or the prey patch ceases its existence due to resource exhaustion, followed by emigration of the remaining prey and the predators.

If we temporarily forget about the resource availability, our previous arguments imply that a (potential) patch is characterized by two state variables taking values in $\mathbb{R}^{+}$: size of prey population $x$ and size of predator population $y$. The deterministic $i$-state movement is given by

$$
\frac{d x}{d t}=\alpha x-\beta y, \frac{d y}{d t}=\gamma y \text {. }
$$

These equations hold for empty patches $(x=y=0)$, pure prey patches $(x>0, y=0)$ as well as for predator patches $(x>0, y>0)$.

The previous description still leaves open the question how empty patches transform into prey patches and prey patches into predator patches: (1.3) leaves the origin as well as the $x$-axis invariant. We shall assume that these transitions are brought about by the random arrival of immigrants recruted from the prey leaving exhausted patches and the predators leaving empty prey patches. However, we are still in for some trouble. When a prey foundress arrives in an empty patch, into what does this patch transform? Our deterministic differential equation models for the local populations in essence are but limits of stochastic models dealing with integer numbers of individuals, the limit being taken by concentrating on densities and letting both the numbers of individuals and the area involved go to infinity. So within the deterministic framework we cannot properly account for the arrival of a single individual having noticeable effects in a finite time. Apparently we run into an incompatibility of our simplifying assumptions! In a biologically consistent model we have to assume that we are dealing with finite numbers of individuals all the time and we have to forsake (1.3) for a much more complicated stochastic model for the behaviour of the $i$-state. Such a model will certainly be intractable. Therefore we shall make a compromise and assume that the arrival of a prey foundress in an empty patch simply sets $x$ equal to one (or $\epsilon$ if you like) and also that the arrival of a predator in a prey patch sets $y$ equal to one, but that from then on (1.3) applies again. 
In our discussion of the patch state we still left the resources out of the consideration. If each patch starts with a fixed amount of resources which are consumed at a rate proportional to the prey density we have

$$
\frac{d r}{d t}=-\delta x
$$

So if no predator arrives a prey patch is exhausted when it has reached age $a_{m}$ defined by

$$
r_{0}=\delta \int_{0}^{a_{m}} x(a) d a=\delta \int_{0}^{a_{m}} e^{\alpha a} d a=\delta \alpha^{-1}\left(e^{\alpha a_{m}}-1\right),
$$

where $r_{0}$ is the amount of resource available in as yet unexploited patches. But the age of a pure prey patch and the prey density in it are monotonically related as $a=\alpha^{-1} \log (x)$. Therefore a patch is exhausted when $x$ reaches the value

$$
x_{m}=\alpha r_{0} / \delta+1
$$

In the same manner we can calculate a boundary line in the $(x, y)$ plane corresponding to the resource exhaustion of the predator patches. For each point in the $(x, y)$ plane corresponds to a unique previous history of prey population size within that patch. So we do not need $r$ as an additional state variable: all the necessary information about the dynamics of $r$ can be expressed in terms of $x$ and $y$.

REMARK 1.11: An easier way to derive (1.5) derives from the observation that

$$
\frac{d r}{d a}+\frac{\delta}{\alpha} \frac{d x}{d a}=0, \quad r(0)=r_{0}, x(0)=1
$$

and therefore for all $a \leqslant a_{m}$,

$$
r(a)+(\delta / \alpha) x(a)=r_{0}+(\delta / \alpha)
$$

which combined with the fact that $r\left(a_{m}\right)=0$ gives (1.5) again.

EXERCISE 1.12: Calculate the resource exhaustion boundary and draw $\Omega$. (Assume that a predator patch is left by the predator as soon as the prey population size drops below one, but not earlier.)

Hint: Extend the method used in the previous remark.

EXAMPLE 1.13: Deterministic binary fission combined with stochastic individual growth.

This example is analogous to the cell kinetics example treated in I.4, except that now we assume that (i) cells divide into two exactly equal parts as soon as they reach size $x$, and that (iia) cell growth is stochastic and, of course, (iib) continuous. (Some motivation for these assumptions has already been provided in remark 1.8). Assumptions (iia) and (iib) together imply that cell size follows a stochastic process of the diffusion type (see e.g. Goel \& Richter-Dyn (1974) or Karlin \& Taylor (1981)), which also allows cell size to decrease. Therefore we need some assumption to prevent cells from becoming too small. The assumption which we shall make here is that cells die on reaching size $x_{0}\left(x_{0}<\frac{1}{2} x_{1}\right)$.

In these notes we intend to concentrate on models in which the continuous $i$-state movements are wholy deterministic. The main role of this example, now and in the future, is to complete the list of possible structural elements and to remind us of the implied unexplored possibilities.

The previous discussion concentrated on the $i$-state process and the reachable $i$-state space $\Omega$. Before going on to the next section a few words are needed still about the corresponding $p$-states. In all cases this is a distribution over $\Omega$, but for the purpose of writing down the p-equation it is often necessary to decompose this distribution into a number of separate components corresponding to the components of $\Omega$.

In examples 1.4 and 1.13, reproduction by binary fission, $\Omega$ is a simply connected subset of $\left(\mathbb{R}^{+}\right)^{k}$ so there is no need to decompose the $p$-state. In example 1.10, the $P P P$ problem, $\Omega$ consists of three separate sets corresponding to respectively the empty patches, the prey patches and the predator patches. Since these sets have different dimensions the same applies to the distribution living on them. If $n=\left(n_{0}, n_{1}, n_{2}\right)$ where $n_{0}$ corresponds to the empty patches, $n_{1}$ to the prey patches and $n_{2}$ to the predator patches, then $n_{0}$ has dimension number of patches (per unit of area, but we shall omit this qualification from now on) $n_{1}$ number of patches per unit of prey density, and $n_{2}$ number of patches per unit of prey density per unit of predator density.

In example 1.1, the invertebrate functional response, the $p$-state also has to be decomposed on dimensional grounds into a component $n_{0}$, corresponding to the searching predators and a component $n_{1}$ corresponding to the predators pursuing or eating prey. Moreover $n_{1}$ makes a sharp jump at the line $\tau=\tau_{e}$ due to the missed strikes. So the $p$-state process can satisfy a partial differential equation only away from this line. 
In example 1.6, colony size distribution in Asterionella, $\Omega$ consists of infinitely many parts with different dimensions. So the $p$-state has to be decomposed into infinitely many components $n=\left(n_{i}\right)_{i=0}^{\infty}$ corresponding to colony
sizes $2^{i}, i=0,1, \cdots$.

Finally in example 1.2, size dependent growth in ectotherms, $\Omega$ is a simply connected subset of $\left(\mathbb{R}^{+}\right)^{2}$ as in example 1.4 (reproduction by binary fission). Yet this example has one peculiarity which sets it apart from all other examples discussed in this section: There is no possible mechanism of dispersion of one cohort. All animals are born at the same size and all animals of the same age share the same feeding history, so their size has remained the same. As a result the $p$-state is no longer a frequency distribution over the full $i$-state space. Instead it is concentrated on some, continually changing, curve in the age-length plane. This fact that the $p$-state necessarily has only a one-dimensional support thwarts our attempt to describe the $p$-state process by a partial differential equation in the usual manner. In section 4.3 we shall therefore develop an alternative formalism in which the $p$-state is described in terms of the agedistribution together with the prevalent age-length relation.

\section{Some modelling philosophy}

In the previous examples the key concept was that of state, of individuals as well as of populations. The reason is that in these notes we wish to stress the mechanistic approach to modelling as opposed to the facile introduction of equations which may look attractive but lack a detailed biological underpinning. Formulating one's model in state space form, usually is a healthy way to bring out one's conception of the physical or biological mechanism one is trying to represent.

In this section we shall give a heuristic introduction to the mathematics of the state concept. We start in the first subsection with, heuristically phrased, definitions of the three allied concepts of state, next state transformation and output map, which are sufficiently general to encompass both varying environments and stochastic behaviour. The terminology in this subsection will be mainly that of the individual but with appropriate modifications everything we say applies to the population level as well. In the next subsection we consider the problem of obtaining state space models capturing the relevant aspects of $i$-behaviour. In the final subsection we shall argue why structured population models as defined in these notes, i.e. models in which the $p$-state is a distribution over an $i$-state space, are the inevitable outcome of a program in which one whishes to explain population dynamics in terms of mechanisms on the individual level.

\subsection{The state concept}

Throughout this book we concentrate on short term causal, as opposed to evolutionary, questions. The main purpose of our modelling effort then is to find such a description of an empirical system, be it an individual or a population, that its future behaviour can be predicted in terms of its initial "preparation" and the intervening environmental conditions. Here behaviour is everything that is of interest to us and that can be measured at least in principle. In the case of populations this may be e.g. numbers or total biomass, in the case of individuals reactions to a prey item, the acts of giving birth or dying, or weight, depending on the needs of the encompassing population model.

We shall start our discussion on the assumption that exact prediction is possible, at least in principle. Later on we shall have a look at stochastic models.

A state is such a collection of (hypothetical or empirically measurable) quantities $X$, that (i) given $X(t)$ and the environmental history (input) between $t$ and $t+\tau, u_{[t, t+\tau)}$, the state at $t+\tau$ is determined by

$$
X(t+\tau)=T_{u[t, t+\tau)} X(t),
$$

where the transformations $T_{u_{[(t+)}}$ satisfy the semigroup property (see figure 2.1.1; we assume that the set of possible input functions $u$ is such that the $u$-segments form a semigroup under the operation "gluing head to tail" (a more formal definition can be found in Metz (1981)), and

(ii) the behaviour of our system at $t$ (the output at $t$ ), is completely determined by the value of $X$ at $t$ and possibly the condition of the environment at the same time. (The relation between state cum environment and corresponding behaviour is called the output map). 


\section{SYSTEM IN STATE SPACE FORM}

$\mathrm{X} \equiv$ state, $\quad \mathrm{U} \equiv$ input

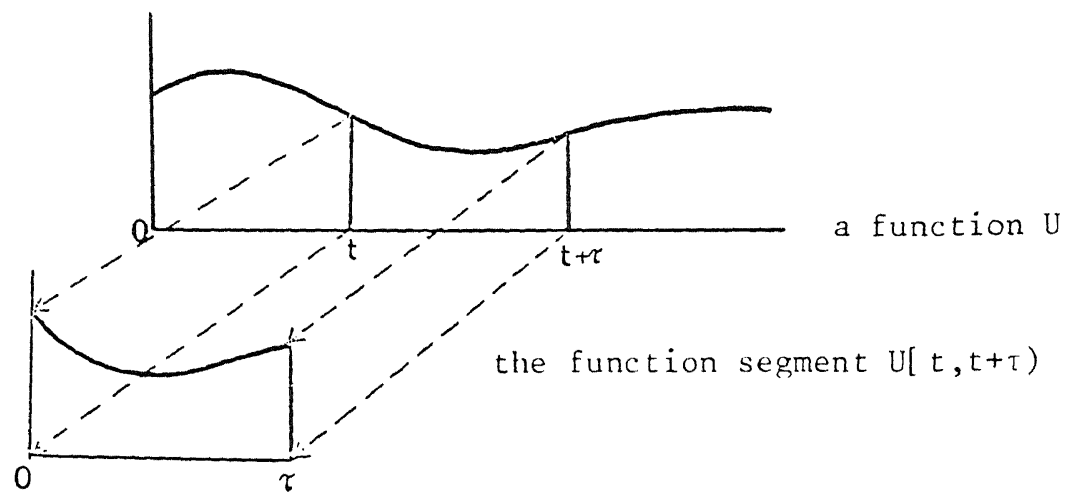

Next state map:

$$
X(t+\tau)=T_{U[t, t+\tau)} X(t)
$$

Semigroup property:

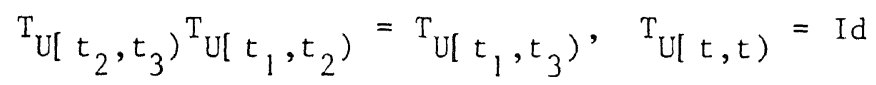

\section{Often with additional requirements:}

(1) $\lim _{\tau \downarrow 0} T_{U[t, t+\tau)} X=X$

(2) $T_{U[t, t+\tau)} X$ continuous in $X$

Fig. 2.1.1. The semigroup property. Id denotes the identity mapping.

Examples are (a) individual size in Daphnia, with food density as the input and individual reproduction, dying from starvation, and possibly feeding rate, as the output (random deaths we still have to leave out of the picture till we are ready to deal with stochastic models as well), and $(b)$ satiation cum maximum time still to be spent handling prey in Holling's mantid, with the (relative) positions of as yet unnoticed prey items as the input, and the catching of a prey item as the output.

The reason to stress a formalism which is sufficiently rich to account for a possibly varying input, is that this enables us to construct models by combining separately constructed building blocks. On the level of the individual one individual's behaviour may be made a component of its own or another individual's input, on the level of the population the population output like total reproduction, total feeding rate or total search rate, may act as a 
component of the input of the same or another population, like that of its prey or its predators. (A detailed discussion of the interface between the individual and population levels will be deferred to subsection 2.3.) When the input is constant our formalism reduces to that of the one-parameter semigroups of next state transformations dealt with in the previous two chapters (with the parameter $t$ the length of the input segment). Some further specialized terminology for autonomous systems may be found in chapter VI. As it is usually next to impossible to reach any interesting conclusions about the behaviour of non-autonomous population systems we shall generally strive to end up with population models in which the subsystems are coupled in such a way that there are not any "loose ends" at the input side.

In general it is very difficult to calculate the operators $T_{u_{u, t+n}}$ explicitly as all kinds of processes interact in deter mining the movement of the state $X$. However, usually these interactions occur only through the changing of the state itself. In that case the contributions of the various processes can be considered separately if $\tau$ is infinitesimally small (and if condition (1) from figure 2.1.1 is fulfilled). Moreover, often for infinitesimally small $\tau$ the total change in $X$ is proportional to $\tau$. (Here we assume implicitly that the state space has a vector (Banach) space structure at least locally; see chapter II.) In that case we can write

$$
\frac{d}{d t} X=A_{v} X
$$

where $A_{v}$ is some operator still depending on the current value of the input $v=u(t)$. The family of operators $A_{v}$ is called the differential generator of the transformation semigroup $\left\{T_{u[t, t+\tau)}\right\}$. It is the differential generators which are our main modelling tool: coupling of subsystems generally is done in terms of differential generators, as is the step from the individual to the population level.

EXAMPLE 2.1.1: In our original Daphnia example from I.3 we assumed that the weight $w$ of an individual changed as a result of two processes, ingestion and basal metabolism. This eventually led to the $i$-differential equation*

$$
\frac{d w}{d t}=\eta^{-1}\left(k \nu f(x) w^{2 / 3}-\zeta w\right)^{+} .
$$

In two animals of the same age the one which has been kept under richer circumstances will have a higher ingestion rate as well as basal metabolism, but this is only due to it being larger; the feeding history is assumed not to have any other direct or indirect effects on present ingestion rate or metabolism.

For the Daphnia population we obtained in 1.3 .2 the $p$-differential equation

$$
\begin{aligned}
& \frac{\partial n}{\partial t}=-\frac{\partial g(x) n}{\partial l}-\mu n \text { for } l \leqslant \bar{l}(x), \\
& n=0 \text { for } l>\bar{l}(x), \\
& g\left(x, l_{b}\right) n\left(t, l_{b}\right)=\int_{l_{b}}^{l_{m}} \lambda(x, l) n(t, l) d l,
\end{aligned}
$$

showing that in our model formulation $x$ as a function of $t$ may act as an input both on the individual and on the population level. At a later modelling stage in I.3.5 $x$ was treated as being coupled dynamically to the Daphnia population again.

REMARKS 2.1.2: (i) Equation (2.1.4) contains a side condition relating the value of the $i$-state distribution at the boundary of $\Omega$ to its values in the interior (equation 2.1.4b)). An operator, like any map, is defined by (a) telling what its domain is and (b) giving a recipe telling how it acts on the elements of its domain. Side conditions form part of the definition of the domain. Their occurrence in the definition of a differential generator $A_{v}$ is typical for infinite dimensional state spaces.

(ii) The $p$-differential generators of the elementary structured population models are always linear. Any nonlinearities are always due to feedbacks through the inanimate or animate environment, i.e. to the coupling of the population input to its or another population's output. Making this explicit is another advantage of our modular approach to model building.

(iii) Generally the basis for arriving at some prospective differential generator $A_{v}$ is entirely heuristic. Therefore it is not $a$ priori clear whether indeed a unique semigroup corresponding to $A_{v}$ exists. We may for example have added an incompatible, or forgotten an essential side condition. Proving existence and uniqueness can be a deep mathematical problem, especially in the nonlinear case (see chapter VI for an example and some references). However, in this chapter we shall put ourselves in the shoes of the biologist and proceed on the assumption that generator and semigroup are well-defined and uniquely related.

- Throughout this chapter we shall follow the notational convention, that arguments of functions will be suppressed when not explicitly needed. 
The definition of the state concept for the stochastic case is exactly analogous to that for the deterministic one except that all the maps involved may be stochastic. As there are some technical difficulties in treating the problem of how to define output maps in full generality, we shall first concentrate on the next state maps. The counterpart of (2.1.1) now is the assumption that for every given present state $X(t)$ and input segment $u_{[t, t+\tau)}$ we are given a so-called transition probability distribution for the resulting state $X(t+\tau)$. In this view the probability distribution for $X(t)$ plays a role which is exactly analogous to the $p$-state, and the transitions probabilities form a semigroup allowing a differential generator in the same manner as we are already used to.

EXAMPLE 2.1.3: If in our mantid model from I.2 or example 1.1 we assume that prey arrive at a rate proportional to their density and proportional to the width of the mantid's search field, we arrive at a stochastic model for an individual predator's behaviour which has prey density instead of prey positions as an input. If we normalize $p$ from 1.2 such that its integral over $s$ equals one it may be interpreted as probability density of an individual predator's satiation, and formula (1.2.5.5) shows us the differential generator of the transition probabilities (also compare exercise 1.2.2.2). The deterministic p-equation as studied in these notes is in this case a direct shadow of the equation applying to the state-transition probability density of individuals, obtained by applying the law of large numbers to a population of independently operating predators.

Generalizing the concept of output map to the stochastic case is a bit more complicated than generalizing that of next state map. First of all there are good reasons to allow the present output to depend not only on the present state and environmental condition as in the deterministic case, but also on the state transition that is presently occurring. For example, in the mantid model from 1.2, with handling times neglected, catches were coincident with state jumps.

REMARK 2.1.4: For models in which all state transitions are deterministic it does not make a difference whether we allow the output to depend on the transitions as opposed to on the state only. For models in which all stochastic transitions are of the jump type the only additional sorts of outputs allowed are coupled to the jumps. The additional possibilities for models with stochastic continuous $i$-state movements are pretty scary. However, in a population dynamical context we only need to consider pure state dependent outputs and outputs which are coupled to jump transitions.

The second point is that we have to allow the output map to be stochastic. Why will become clear in a few lines when we have discussed how this statement should be interpreted. Consider for the time being a hypothetical situation in which both environment and state stay constant for a while. If we allow at each time point one of a number of possible output values to be chosen randomly, our animal would switch its behaviour infinitely often in any small time interval, unless we assume that the output map is such that one particular output value is chosen with probability one. Yet, as each time interval infinitely many choices are made, this assumption does not preclude the occurrence of other possible output values as long as these occurrences are point events having zero duration. These point events necessarily occur totally at random, or, in the language of probability theory, in independent Poisson processes, the concept of probability of occurrence of an output value (or range of output values) being taken over by that of the rate of the corresponding Poisson process. (where rate in this case may be defined as the mean number of occurrences per unit of time). Now assume that state and environment vary over time. This means that the rates of the Poisson processes start to vary as well, in a manner which deterministically depends on the state cum environment. This then is the concept of "stochastic output map" we need. An example is provided by the idea of an age dependent birth rate, the births themselves being modelled as randomly occurring point events. (Ask yourself what state space we would need in this case if we only allowed deterministic output maps!)

Having indicated the two main extensions of the concept of output map to the case of stochastic systems we leave it as an exercise to work out the various possible, and often mindboggling, combinations.

\subsection{Obtaining an $i$-state representation}

We shall confine ourselves here to $i$-state spaces which consist of subsets of $\mathbb{R}^{k}, k=0,1,2, \cdots$ as these are the only ones playing a role in these notes. We shall indicate the $i$-state as $X$.

The easiest case, experimentally as well as conceptually, occurs when $X$ wholly consists of physiological or physical quantities allowing direct measurement at least in principle. Examples are weight, amount of a toxic chemical present in the individual, fat reserves, gut content, etc. $X$ is then uniquely determined, at least in principle and it is usually rather simple to write down a differential generator for it on the basis of its interpretation. Similarly it is not difficult to specify the value of $X$ at the moment of birth or after a state jump. For practical reasons we should choose as 
simple an $i$-state representation as possible, i.e. we should identify states which do lead to the same behaviour now and in the future (minimal dimension of the $i$-state space) and we should confine the attention to those states that can be reached during the population situations that have our interest (the reachable $i$-state space $\Omega$ ), but that is all there is to it. Here behaviour again means everything that is population dynamically relevant like giving birth, dying, capturing prey, etc.

Life becomes more complicated if on the individual level we have only input-output data at our disposal like the observed moments of molting or egglaying as a function of the temperature regime, or the observed reactions to prey items as a function of the feeding history. In that case we have to dream up an $i$-state representation satisfying our requirements (i.e. (i) and (ii) from the previous subsection). Examples of state variables of an input-output provenance are physiological age, degree of developmental commitment, satiation (as opposed to the physiological variable gut content with which it is frequently and uncritically identified; see e.g. Doucet \& van Straalen (1980)) etc.. If our individuals are totally deterministic and if we can do all input-output experiments that we want, then systems theorists have proved that under very general conditions it is possible to construct a state space representation generating those input-output data and that moreover all minimal state space representations that do the same job are equal up to a choice of parametrization (see e.g. Metz $(1977,1981)$ for a review). Minimal here means again that there are no behaviourally indistinguishable states, and that all states can be reached given suitable inputs. However in practice the number of experiments necessary to reach uniqueness is forbidding except in the simplest cases. Therefore we have to satisfy ourselves with educated guesses. Moreover, there is the problem of extending our model to environmental circumstances not subsumed under the original experiments.

It should be remarked that "deterministic" refers here to the "internal" determinism of the individual machinery. The total $i$-state process may well be stochastic due to stochastic but $i$-experimentally observable inputs. Holling's mantid again provides an example. The mantid itself is totally deterministic; all stochastic events can be ascribed to prey behaviour, which can be considered an input quantity as far as the construction of the $i$-model is concerned.

The uniqueness theorem no longer holds when the $i$-process is stochastic "internally". This means that it is possible to construct two models which assume a totally different physiology but still predict exactly the same behaviour. Therefore in the stochastic case input-output based state space representations do not have the same epistemological status as those based on exactly reproducible input-output experiments. An example of such a "weak" state space representation is the use of age to model the change over time of an individual's birth rate or probability of dying.

EXAMPLE 2.2.1: Under constant circumstances both the stochastic cell growth model from the previous section (example 1.13) and the simple Daphnia model from 1.3 each allow equivalent age representations. However in the Daphnia example there is a one to one correspondence between age and size whereas no such relation exists in the stochastic individual growth case. Both size dependent model formulations allow rather simple extensions to varying nutritional conditions, whereas this is not the case for the age dependent models.

REMARX 2.2.2: The stochastic fine structure of individual behaviour is often largely irrelevant to the gross behaviour of a deterministic structured population model derived from it. This sometimes makes it possible to replace an $i$-model by a population dynamical equivalent $i$-model of a simpler kind. This phenomenon still bears closer study. At present only scattered examples can be found in the literature, mainly pertaining to age-dependent models. The following example of our own making may illustrate the idea. In chapter IV, on age dependence, we shall consider some other examples when discussing the relationships between various kinds of equations describing population behaviour. If we change the stochastic cell growth model from example $1.13 \mathrm{a}$ bit by assuming that an individual divides into unequal parts the sizes of which bear a constant relation to each other, we may speak of an individual giving birth each time it hits the division threshold and at the same time falling back in size by a fixed amount. The two population dynamically relevant output quantities are being alive (or dying) and giving birth. We shall also assume that the environment as perceived by the individuals is constant so that their behaviour does not depend on absolute time or on each other.

Now assume that we do not have our physiologically based model. We then can construct an $i$-state space representation on a purely behavioural basis by defining the state of an individual to be the best possible summary of previous behaviour as far as the prediction of future behaviour is concerned. The state space of this representation is $\mathbb{R}^{+} \times\{0,1\}$, where the second component tells us whether the individual is nulliparous or that it already has given birth at least once, and the first component tells us the time since the last event in its life, whether it be being born or giving birth. Such a model is behaviourally equivalent to our original physiological model. By its very construction knowing the frequencies of the individuals in the various states of our behaviourally based representation gives us a prediction of the future population dynamical constributions by these individuals which is the best possible one if we have nothing else to go by than their externally observable behaviour. (Knowing the frequencies of their sizes would probably give a, slightly, better prediction.) 
The reason why we did not end up with age, i.e. time since birth, as the only state variable, is that the events of an individual dying and giving birth are dependent: when an individual has just given birth we know it is not near to the lower, death, boundary of its physiological state space. Yet, a model in which we use age as the only descriptor of an individual, while not a genuine model of its behaviour, can do almost as good a population dynamical job as our more complicated model, given that we match both the probability distribution of the age at death, and the mean rate of giving birth (conditional on the individual being alive) to those of our other two $i$-models. The only difference between the deterministic population models based on the three $i$-models is that the more detailed models will be better in predicting births and deaths occurring in the remainder of the founder population. But once this ancestral population is all gone all three models behave exactly the same as far as births, deaths and total population number are concerned.

In practice most $i$-state space representations will be based on a mixture of physiological and input-output considerations, the exact procedural details being dependent on the experimental possibilities particular to the case. Good examples are provided by the work of Kooijman and of Sabelis reported in part B of these notes.

\subsection{From the individual to the population level}

The pervading theme in these notes (with the possible exception of chapter IV) is that (i) from a reductionist/mechanistic point of view the sensible choice for the $p$-state should take the form of an $i$-state distribution, and (ii) we wish to formulate our models in terms of p-equations which (iii) should be based, either directly or indirectly, on the law of mass action. Yet in our presentation up till now the status of (i) to (iii) was to a large extent that of articles of faith, as the argument presented in I.1 was at best rather sketchy. In this subsection we intend to fill in some of the gaps.

\section{The p-state}

From a mechanistic viewpoint the purpose of our modelling effort should be the explanation of the behaviour of populations in terms of the doings of the individuals constituting them. Therefore our models should keep track of those doings at least to such an extent as is necessary for calculating the population properties in which we are interested. These properties can for example be numbers of individuals, (or rather densities, i.e. number per unit of area or volume, see remark I.1.1 (ii)) total biomass etc. To simplify the argument we shall from now on assume that the only properties in which we are interested are total numbers. Individuals therefore only have to be distinguished insofar they have different prospects of contributing to changes in the numbers of their own kind or of any other population in which we are interested, either directly, e.g. through catching prey, giving birth, being caught by a predator or migrating, or indirectly by influencing resource availability etc. This shows that our $p$-state should at least contain complete information about the frequency distribution of the $i$-states. The question is under what conditions this information is also sufficient.

For the $i$-state distribution to be sufficient information clearly a first (1) necessary condition is that individuals do not differ in influences on their behaviour other than through their $i$-states. Therefore we have to assume that they all experience the same inputs. This leads us to define a population as a collection of individuals all experiencing the same circumstances, be it "physical" circumstances like temperature or resource availability, or "biotic" circumstances like prevailing prey or predator densities. (The mantid example makes clear how we should (re)phrase our $i$-models such that they have densities (in the spatial sense) of other individuals as their input. That we have to phrase our $i$-models this way is a consequence of condition (2) below.) This means that by definition the environment of the population and that of the individuals is the same thing, and also that the environmental circumstances are considered as extraneous to the population. They should be generated by some other part of our model or be given a priori, e.g. by telling that the prey are just the smaller and the predators are just the larger members of the same population, or by giving the densities of prey and predators as functions of time.

REMARKS 2.3.1: (i) To conform to the usual biological interpretation of population we shall also wish to add to our definition that the individuals belong to the same "species", i.e. that any two individuals are equivalent in the sense that at least one member of the pair is equal in all relevant aspects to a potential descendant of the other member of the pair.

(ii) If the environmental circumstances cannot be considered the same for all individuals we wish to deal with, then the total or meta-population should be split into subpopulations, e.g. on the basis of location in space, such that within each subpopulation the circumstances are comparable. If we characterize individuals with an extended "type"label consisting of a "location" label plus an $i$-state label, we can write down $p$-equations for the meta-population by going through exactly the same kind of routines as when writing down an ordinary p-equation. 
(iii) The linearity of our elementary $p$-equations is due precisely to our equating of population and individual inputs.

A second (2) necessary condition for the $i$-state distribution to qualify as a $p$-state is that the population output can be calculated from it. This is by definition the case for any population properties we set out to explain. However, it should also apply to those outputs which we need as inputs for other parts of our model. Therefore the impact of individuals on their environment, be it physical or biotic, should only be through their "mass action", i.e. it should be possible to express this impact in terms of the spatial densities of the individuals in various $i$-states, without taking account of the behaviour of the separate individuals as individuals.

Together conditions (1) and (2) are also sufficient for the $i$-state distribution to qualify as $p$-state.

REMARX 2.3.2: (i) Biologically (1) and (2) mean that the individuals either do not interact at all, or if they do so, "mix homogeneously". This assumption will often apply as a first approximation to animals and also to plants interacting through a fast mixing chemical environment, but not for example to plants interacting with a small number of fixed neighbours through mutual shading.

(ii) When individuals sometimes temporarily form closely knitted groups but otherwise mix randomly we can still conform to (1) and (2) by a suitable redefinition of what we call an individual. An example is provided by the Asterionella colonies from example 1.6.

Before going on to the next topic, determinism, we still have to voice a warning. Even though a framework in which the elements are populations which are only coupled through their inputs and outputs in the sense of (1) and (2) is essentially sound, a too rigorous adherence to it may lead to the impractical consequence that we have to duplicate parts of our $p$-equations when interactions between individuals cannot be considered point events: For example, what we called pursuing predators in the invertebrate predator example 1.1 are in fact predator-prey pairs. So the part of the predator $i$-state-space that corresponds to pursuit (see fig. 1.1) also appears as part of the prey $i$-statespace, and the component of the predator $p$-state representing the pursuing predators exactly equals the component of the prey $p$-state representing pursued prey. Clearly it is more practical to treat the predator-prey pairs locked in pursuit as one, new, population in addition to the populations of single prey and predators.

\section{p-equations and determinism}

The use of a differential equation as a means of generating population behaviour is independent upon the assumption that our populations behave in a deterministic fashion. Biologically this means that (3) the number of individuals in the population is sufficiently large that any chance processes on the individual level are ironed out, and (4) all essential environmental conditions which are not outputs of the $p$-systems comprising our model are given as deterministic functions of time.

REMARK 2.3.3: (i) In checking (3) for spatially distributed populations as introduced in remark 2.3.1 (ii) we have to distinguish two cases. When the location label is discrete all subpopulations should be sufficiently large. However, in the case of a continuous location label (like positional coordinates in $\mathbb{R}^{2}$ ) it is only necessary that large numbers of individuals are present in the largest areas over which the environmental circumstances do not change overmuch. For direct biotic interactions depending on relative distances this condition is equivalent to: for all individuals the number of individuals potentially capable of interacting with them within a short time interval is large.

(ii) In our phrasing of assumptions (1) and (2) we already anticipated on the assumption of determinism, or, more particularly, on assumption (3). When numbers of individuals are not infinite the behaviour of one population can also show a dependence on the behaviour of another population, which cannot be captured in terms of a dependence on the state of that other population only: we have to allow for a dependence on state transitions as well. For example, in a model for predator-prey interaction dealing with finite numbers of individuals we have to account for the fact that the capturing of a prey by a predator is exactly coincident with a drop in the prey number. This is a stronger coupling of the two population processes than just a dependence of the dynamics of the prey population on the satiation distribution of the predators combined with a dependence of the dynamics of that satiation distribution on the prey density. But in large populations we soon loose sight of this kind of coupling at the level of the microscopic probabilistic structure.

\section{The law of mass action}

The "law of mass action" states not only that the rate per unit of area/volume at which a reaction occurs depends on no more than the spatial densities of the two reactants, but also that this rate is linear in the densities of each of them (with the understanding that if the two reactants are of the same kind the reaction rate is quadratic in the reactant density). 
The law of mass action is a direct consequence of the assumptions that (i) two identical reactant individuals have the same probability per unit of time to undergo a reaction, where (ii) this probability depends only on the density of the other individuals, if any, involved in the reaction (and possibly on the physical circumstances), and moreover (iii) the maximum number of individuals involved in any reaction is two. Adding up over all identical individuals in a unit of volume gives us the expected number of reactions per unit of time, the reaction rate.

A more careful analysis of the previous argument shows that there is an intimate connection between the law of mass action and the concept of $i$-state. (i) follows from the fact that two individuals belonging to the same population and being in the same $i$-state should certainly have the same probability of reacting. After all the concept of $i$-state was introduced precisely to guarantee this kind of behavioural equivalence. The reasoning showing that the probability of reacting may only depend on the density of the individuals with which it reacts and not on the densities of other sorts of individuals is more complicated. We start with the observation that whitin our framework reactions cannot have a duration, for if an interaction between individuals or a behavioural transition costs time, this time has to be accounted for in terms of the $i$-state space (compare our discussion of the invertebrate predator in example 1.1) Next we throw in the assumption (A) that within a finite time interval an individual can only interact with finitely many other individuals (see also remark 2.3 .4 (iii) below). Therefore the only reactions in which (a) other individuals are involved and which (b) have a noticeable effect, are births, deaths and $i$-state jumps. Only the third type of events results in a protracted effect on an individual's behaviour, but only through a change in its $i$-state. The direct involvement of any other individual in the generation of a reaction automatically classifies that individual as a reactant in the sense of the law of mass action, thus precluding any direct effect of non-reactants in the probability per unit of time that an individual in a given state reacts. Finally (iii) follows from a stronger version of our earlier assumption (A): (A ') for any individual in any condition the probability per unit of time of interacting with an other individual is finite.

Yet there exist apparently reaction rates which do not conform to the law of mass action. Our discussion of the invertebrate functional response in 1.2 indicates how such reaction rates may be fitted into the general framework by paying attention to faster time scales and correspondingly more complicated $i$-state spaces. Note, however, that that time scale argument was based essentially on the prey population being much larger than the predator population. It is only in the limit when the ratio between prey and predator densities has gone to infinity that we obtain nothing more complicated than a nonlinear relation between overall predation rate and (rescaled) prey density. But in taking this limit we gave up treating the predator and prey populations on equal footing.

REMARK 2.3.4: (i) The reason to stress in the present context the per unit of area/volume aspects is that an individual cannot "see" the total number of individuals present with which it can react, but only the local density of individuals surrounding it (compare remark I.1.1 (ii)).

(ii) For the initial argument leading up to the law of mass action it is already sufficient that the expected number of reactions undergone by individuals are equal. This is the basis for the construction of population dynamically equivalent simplified $i$-models as discussed in remark 2.2.3.

(iii) Our discussion of the invertebrate functional response in 1.2 also shows how an apparently continuous $i$-movement may result from very many interactions each having a very small effect. Again this result was reached by a procedure which precludes treating the prey as a population of individuals au par with that of the predators.

\section{Mass balance}

In this section we shall discuss the various building blocks of the differential generator for the $p$-state transitions. The whole problem of specifying this differential generator boils down to an appropriate accounting of $p$-mass: individuals may be assumed to (dis)appear only through known (or at least named) causes like births, deaths or transport to or from some other place in the reachable $i$-state-space $\Omega$. Moreover, during infinitesimally small time intervals these processes act independently. Therefore we can specify the $p$-differential generator by simply adding the various separate contributions to the local change of the $p$-mass. Depending on whether we concentrate on $i$-states inside or at a boundary of $\Omega$ our accounting gives us components of the defining formula of the $p$-differential generator $\mathrm{A}$ or components of a side- condition at that boundary. Since our discussion will be in terms of the biological processes involved we shall be intermittently dealing with one or the other.

First we introduce some notation. We start with repeating that throughout this chapter we shall adhere to the

CONVENTION: we shall suppress any arguments of functions that are not explicitly operated on or referred to. 
The $i$-state will be denoted as $x=\left(x_{1}, \cdots, x_{k}\right)^{T}, k$ the dimension of the component of $\Omega$ under consideration." The density of individuals at $x$ we shall indicate as $n(x)$, the $p$-state as $n$, i.e.

$$
n(x) \Pi d x_{i} \equiv \text { number of individuals in the infinitesimal rectangle } \Pi\left(x_{i}, x_{i}+d x_{i}\right)
$$

or, if your inclination is mathematical instead of scientific, for any $\Omega_{0} \subset \Omega$ :

$$
\int_{\Omega_{0}} n(x) d x \equiv \text { number of individuals in } \Omega_{0} \text {. }
$$

(where $d x$ denotes Lebesgue measure ${ }^{\dagger}$ ).

In the interior of $\Omega$ the differential generator $A$ can be written as

$$
\frac{\partial n}{\partial t}=\delta_{t} n+\delta_{s}+n-\delta_{s-n}
$$

where

$\delta_{t} n \equiv$ contribution to the local change due to continuous $i$-state movement (the $t$ stands for transport),

$\delta_{s}+n \equiv$ contribution to the local change due to births or jumps from elsewhere in $\Omega$ (the $s$ stands for source)

$-\delta_{s}-n \equiv$ contribution to the local change due to deaths or jumps to elsewhere in $\Omega$ (the $s$ stands for sink) In principle

$$
\delta_{t} n=\delta_{t d} n+\delta_{t s} n
$$

where

$\delta_{t d} \equiv$ contribution of the deterministic (local average) component of the continuous $i$ - state movement

$\delta_{t s} n \equiv$ contribution of the purely stochastic component of the continuous $i$-state movement

However, we shall conventionally assume that $\delta_{t s} n=0$, at least in the main body of the chapter, deferring to appendix B the discussion of the complications that arise when $\delta_{t s} n \neq 0$. Also, when concentrating on one particular kind of contribution we shall often without further notice assume that all other contributions are zero.

Side conditions can be decomposed in essentially the same manner as the defining formula for A and the same conventions will apply.

The various standard notational conventions of vector analysis and calculus in $\mathbb{R}^{k}$, as well as a discussion of the underlying concepts geared towards a biological audience, can be found in appendix A.

- We shall think of $x$ as a column vector. ${ }^{T}$ denotes transposition.

If If you are not familiar with the concept of Lebesgue integration
dix A tries to convey the idea behind the concept in a manner geared to the minimal use made of it in this chapter. 


\subsection{Mass transport due to continuous $i$-state movement}

This subsection is divided into two parts. In the first part we follow the biologists line of approach, with the stress on the heuristics and minimizing the algebra. In the second part we essentially repeat the argument in a manner to suit the taste of a mathematician.

\subsubsection{A biologist's shortcut}

In chapter I we derived that the one dimensional conveyor belt is described by

$$
\delta_{t} n=-\frac{\partial}{\partial x}(v n)=-\left(v \frac{\partial n}{\partial x}+n \frac{\partial v}{\partial x}\right)
$$

where $v$ denotes its local speed (the speed of the $i$-movement). For $k>1$ we can just add the contributions of the transport in the various directions, i.e.

$$
\delta_{i} n=-\sum_{i} \frac{\partial}{\partial x_{i}}(v n)
$$

where $v_{i}$ denotes the speed of movement in the direction of the positive $x_{i}$ axis. Using the notation from vector analysis this can also be written as

$$
\delta_{t} n=-\nabla \cdot(v n)
$$

where $v$ denotes the vector of local speeds.

The previous line of argument concentrated on one locality in $\Omega$. Some more insight in the nature of (3.1.2) can be gained by rederiving it in a different manner. To this end we imagine that we move along through $\Omega$ with the flow generated by the vector field $v$, or more biologically phrased, that we stick to one individual and observe what happens to the content of a little box around it, currently of the form $\Pi\left(x_{i}, x_{i}+d x_{i}\right)$, and having a volume $\Pi d x_{i}$. At $t+d t$ this box has been transferred to a position slightly more downstream and has changed in shape and therefore also in volume (see fig. 3.1.1). The new position is given by $x \mapsto x+v d t$, the new value of the $i$ 'th state variable being $(x+v d t)_{i}=x_{i}+v_{i} d t$. To calculate the volume of our little box after its transfer downstream we start by noting that the map transforming neighbourhoods of $x$ into neighbourhoods of $x+v d t$ may to first order in the $d x_{i}$ be approximated by a linear map. This linear map transforms the original square box into a parallelepipid. Therefore any curviness seen in fig. 3.1.1 can safely be neglected as being due to higher order terms in the $d x_{i}$ only. Next we concentrate on one $i$-state variable at a time, say the $i$-th, i.e. we consider the dilation of the box in the direction of the $i$-th unit vector $e^{(i)}$, while temporarily neglecting any changes in the other sides of the box. To this end we look what happens to the two adjacent corners $x$ and $x+e^{(i)} d x_{i}$. The first corner transforms into $x+v(x) d t$, and the second one into

$$
x+e^{(i)} d x_{i}+v\left(x+e^{(i)} d x_{i}\right) d t=x+e^{(i)} d x_{i}+\left(v(x)+\frac{\partial v}{\partial x_{i}} d x_{i}\right) d t .
$$

Apparently under our linear map $e^{(i)} d x_{i} \mapsto\left(e^{(i)}+\frac{\partial v}{\partial x_{i}} d t\right) d x_{i}$ only the change in the $e^{(i)}$ direction contributes to the volume change (see fig. 3.1.2A (or B)). 


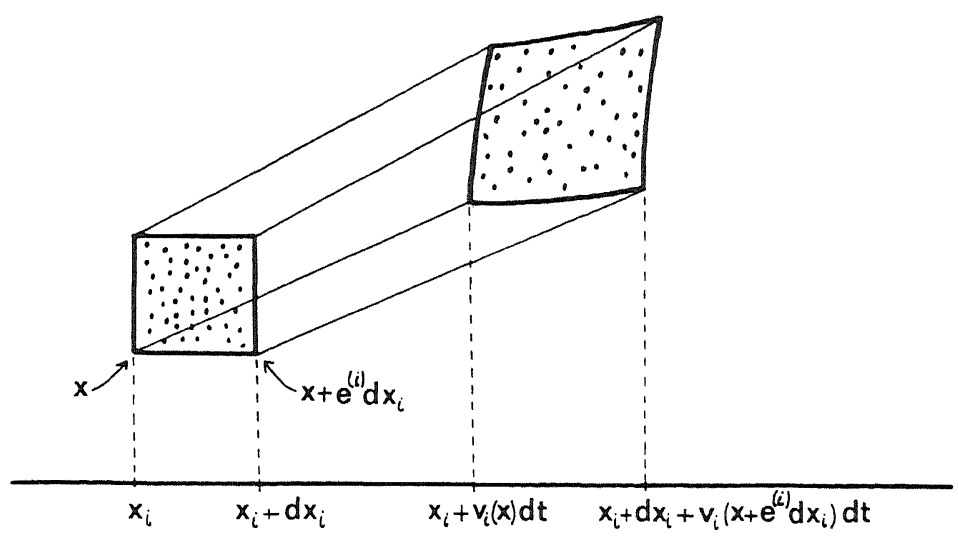

Fig. 3.1.1. The change of an infinitesimal volume when it moves along with the stream, and the contribution to this change due to changes in the $i$-th state variable.

Singling out the $i$-th coordinate we find $\left(e^{(i)}+\frac{\partial v}{\partial x_{i}} d t\right)_{i} d x_{i}=\left(1+\frac{\partial v_{i}}{\partial x_{i}} d t\right) d x_{i}$. Therefore the dilation of our box in the direction of the $i$-th coordinate adds a fractional increase of $\frac{\partial v_{i}}{\partial x_{i}} d t$ to its volume. If, as before, we neglect higher order terms we can simply add up the contributions of the volume changes in the directions of the various $i$-state variables (see fig. 3.1.2C, and/or exercise 3.1.1) to arrive at a total fractional volume change of $\sum_{i} \frac{\partial v_{i}}{\partial x_{i}} d t$. Equating the numbers of individuals in the box before and after its infinitesimal transformation then gives

$$
n(t+d t, x+v d t) \Pi d x_{i}\left(1+\sum \frac{\partial v_{i}}{\partial x_{i}} d t\right)=n(t, x) \Pi d x_{i}
$$

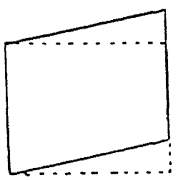

A

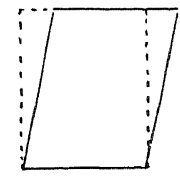

B

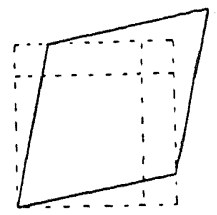

C

Fig. 3.1.2. Decomposing the infinitesimal volume change of the box from fig. 3.1.1 into the component changes along the axes.

Hence

$$
[n(t+d t, x+v d t)-n(t, x+v d t)]+[n(t, x+v d t)-n(t, x)]+n(t+d t, x+v d t) \sum \frac{\partial v_{i}}{\partial x_{i}} d t=0
$$

Dividing by $d t$ finally gives 


$$
\frac{\partial n}{\partial t}+\sum \frac{\partial n}{\partial x_{i}} v_{i}+n \sum \frac{\partial v_{i}}{\partial x_{i}}=0
$$

which is equivalent to (3.1.1).

The various terms in (3.1.3) bear special names referring to their interpretation. The quantity

$$
n \sum \frac{\partial v_{i}}{\partial x_{i}}=n \nabla \cdot v
$$

is called the dilation (or dilatation) since it accounts for the contraction or expansion of our volume element $\Pi\left(x_{i}, x_{i}+d x_{i}\right)$. The remaining quantity

$$
\sum \frac{\partial n}{\partial x_{i}} v_{i}=\nabla n . v
$$

accounts for the pure transportation of individuals with the $i$-flow and therefore is called the convection term. (compare the discussion after (I.2.5.4)). Finally the quantity

$$
\frac{D n}{D t} \stackrel{\text { def }}{=} \frac{\partial n}{\partial t}+\nabla n \cdot v
$$

is called the material or substantial derivative as it describes what happens around one individual which we follow through its life (see also section 4 on integration along characteristics).

As a final point it should be noticed that $v$ in (3.1.1) to (3.1.3) may depend explicitly or implicitly on time, e.g. as a result of a dependence on the state of other or the same populations. This does not influence at all our derivation of these formulae.

EXERCISE 3.1.1: Show that the linear map transforming a neighbourhood of $x$ into a neighbourhood of $x+v d t$ allows the matrix representation $I+D v d t$, where the derivative $D$ is taken with respect to $x$. Therefore the volume of our little box after transformation equals $\Pi d x_{i} \operatorname{det}(I+D v d t)$. Show that this is equal to $\Pi d x_{i}\left(1+\sum \frac{\partial v_{i}}{\partial x_{i}} d t+O\left(d t^{2}\right)\right)$.

EXERCISE 3.1.2: Write down the transport part of the p-differential generators of examples 1.1, 1.2, 1.4, 1.6, and 1.10 from section 1. (Mind the decomposition of $\Omega$ !)

\subsubsection{The mathematician's derivation}

The more rigorous derivation of the transport component of the $p$-differential generator to be presented in this subsection uses the physically inspired concept of flux, which will not be introduced in intuitive terms untill well into subsection 3.2.2. So if you are not familiar with this concept already, you are advised first to read on and only afterwards to return to this subsection.

Just as with the "derivation" in subsection 3.1.1. there are two possible approaches depending on whether we imagine ourselves as staying put at a fixed point in $\Omega$ or as moving along with the stream. These two approaches are called after the mathematicians Euler and Lagrange respectively. The Euler approach has the advantage that it still applies in situations where the flux vector $\phi$ depends in a more complicated manner on the local density than just $\phi=v n$ (e.g. due to random components in the continuous $i$-state movement), but the Lagrange approach adds more to our insight into the nature of the transport equation and its solutions.

The ideas presented below arose in the context of continuum mechanics. We refer to Lin \& Segel (1974), Segel (1977 a, b) for an introduction to this subject. Also see Aris (1978).

\section{The Euler approach}

Let $\Omega_{0}$ be a domain in $\Omega$ with a piecewise smooth boundary $\partial \Omega_{0}$. If $\phi$ denotes the flux vector and $\nu$ the outward normal at a point $x$ on $\partial \Omega_{0}$, the outward flux at $x$, be it positive or negative, is given by $v \phi$. Conservation of mass implies that

$$
\frac{d}{d t} \int_{\Omega_{0}} n d x+\int_{\partial \Omega_{0}} \nu \cdot \phi d \sigma=0
$$

where $\sigma$ denotes Lebesgue measure on $\partial \Omega_{0}$. Applying the divergence theorem we find

$$
\frac{d}{d t} \int_{\Omega_{0}} n d x+\int_{\Omega_{0}} \nabla \cdot \phi d x=0
$$


or

$$
\int_{\Omega}\left(\frac{\partial n}{\partial t}+\nabla \cdot \phi\right) d x=0 .
$$

Since $\Omega_{0}$ is arbitrary this is equivalent to (by the so-called Dubois-Reymond lemma)

$$
\frac{\partial n}{\partial t}+\nabla \cdot \phi=0
$$

and in particular when $\phi=v n$, as is the case when the continuous $i$-state movements are all deterministic (see subsection 3.2.2),

$$
\frac{\partial n}{\partial t}+\nabla \cdot v n=0
$$

\section{Langrange approach}

The calculations for the Lagrange approach are considerably more complicated. However, the technicalities involved also have some interest in themselves. For example, in part B both Aldenberg and Roots use calculations of this kind in the context of concrete biological examples.

As said before, the Lagrange approach is based on following an individual and looking at what happens in its immediate vicinity. Let such an individual be characterized by its $i$-state $x_{0}$ at $t=0, x_{0}$ in the interior of $\Omega$. Its $i$ state at $t$ can be determined from the $i$-equation

$$
\frac{d x}{d t}=v(t, x) \quad x(0)=x_{0} .
$$

We assume that (3.1.10) allows a unique solution which can be written as

$$
x\left(t, x_{0}\right)=T_{[0, t)} x_{0}
$$

where $T_{[0, t)}$ is the next $i$-state operator acting over the interval $[0, t)$. The uniqueness of $x\left(t, x_{0}\right)$ guarantees that $T_{[0, t)}$ allows an inverse $T_{(0, t)}^{-1}$ at least in a neighbourhood of $t=0, x=x_{0}$.

Now consider an arbitrary domain $\Omega_{0}(0)$ in the interior of $\Omega$, and let

$$
\Omega_{0}(t):=\left\{x\left(t, x_{0}\right) \mid x_{0} \in \Omega_{0}(0)\right\} .
$$

With this notation the conservation of individuals expresses itself as

$$
\int_{\Omega_{0}(t)} n(t, x) d x=\int_{\Omega_{0}(0)} n(0, x) d x
$$

This relation forms the basis of the Lagrange derivation of the transport equation. But before we proceed to the derivation we introduce some auxiliary notation

$$
\rho\left(t, x_{0}\right):=n\left(t, x\left(t, x_{0}\right)\right), J\left(t, x_{0}\right):=\operatorname{det} D_{x_{0}} x\left(t, x_{0}\right)=\operatorname{det} D T_{[0, t)} x_{0} .
$$

(NB The function $\rho$ is not a density! If one wishes to transform variables and keep the interpretation of densities one should include the Jacobian of the transformation, in this case $J$.) To calculate $J$ we define the matrix valued function $M$ by

$$
M\left(t, x_{0}\right):=D_{x_{0}} x\left(t, x_{0}\right)=D T_{[0, t)} x_{0} .
$$

By differentiating (3.1.10) for $x_{0}$ we find

$$
\frac{d M}{d t}=D_{x} v\left(t, x\left(t, x_{0}\right)\right) M, \quad M(0)=I \text {. }
$$

This is a linear time varying o.d.e. Elementary linear algebra (see appendix A) shows that $\operatorname{det} M$ satisfies

$$
\frac{d J}{d t}=\left(\operatorname{trace} D_{x} v\right) J=(\nabla \cdot v) J, \quad J\left(0, x_{0}\right)=1
$$

and therefore

$$
J\left(t, x_{0}\right)=\exp \left[\int_{0}^{t} \nabla \cdot v\left(\tau, x\left(\tau, x_{0}\right)\right) d \tau\right]>0 .
$$

(The last inequality also shows that for $v$ differentiable in $x$ the inverse map $T_{[0, t)}^{-1}$ exists at least in a neighbourhood of 
$t=0, x=x_{0}$.)

Having introduced all necessary instruments we are ready to derive the transport equation. To this end we shall calculate $\frac{d}{d t} \int_{\Omega_{0}(t)} n(t, x) d x$ by first applying the transformation $x=T_{[0, t)} x_{0}$ and then use the inverse map $T_{[0, t)}^{-1}$ to transform back again. Owing to the positivity of $J$ we can drop the absolute value signs.

$$
\int_{\Omega_{0}(t)} n(t, x) d x=\int_{\Omega_{0}(0)} n\left(t, x\left(t, x_{0}\right)\right) J\left(t, x_{0}\right) d x_{0}=\int_{\Omega_{0}(0)} \rho\left(t, x_{0}\right) J\left(t, x_{0}\right) d x_{0} .
$$

Therefore

$$
\begin{aligned}
0 & =\frac{d}{d t} \int_{\Omega_{0}(t)} n(t, x) d x=\int_{\Omega_{0}(0)}\left\{\frac{\partial \rho}{\partial t}\left(t, x_{0}\right) J\left(t, x_{0}\right)+\rho\left(t, x_{0}\right) \frac{\partial J}{\partial t}\left(t, x_{0}\right)\right\} d x_{0} \\
& =\int_{\Omega_{0}(0)}\left\{\left[\frac{\partial}{\partial t} n\left(t, x\left(t, x_{0}\right)\right)+\nabla n\left(t, x\left(t, x_{0}\right)\right) \cdot v\left(t, x\left(t, x_{0}\right)\right)\right] J\left(t, x_{0}\right)+n\left(t, x\left(t, x_{0}\right)\right) \nabla \cdot v\left(t, x\left(t, x_{0}\right)\right) J\left(t, x_{0}\right)\right\} d x_{0} \\
& =\int_{\Omega_{0}(0)}\left[\frac{\partial}{\partial t} n\left(t, x\left(t, x_{0}\right)\right)+\nabla n\left(t, x\left(t, x_{0}\right)\right) \cdot v\left(t, x\left(t, x_{0}\right)\right)+n\left(t, x\left(t, x_{0}\right)\right) \nabla \cdot v\left(t, x\left(t, x_{0}\right)\right)\right] J\left(t, x_{0}\right) d x_{0} \\
& =\int_{\Omega_{0}(t)}\left\{\frac{\partial n}{\partial t}+\nabla n \cdot v+n \nabla \cdot v\right\} d x=\int_{\Omega_{0}(t)}\left(\frac{\partial n}{\partial t}+\nabla \cdot(n v)\right) d x
\end{aligned}
$$

which again implies (3.1.9).

The next two exercises provide some links with Aldenberg's use in part B of the flux in deriving the balance law.

EXERCISE 3.1.3: Consider the special case that $\Omega$ is 1-dimensional and $v$ time independent. Show that $J\left(t, x_{0}\right)=v\left(x\left(t, x_{0}\right)\right) / v\left(x_{0}\right)$.

Hint: Use that $\frac{d}{d t} v\left(x\left(t, x_{0}\right)\right)=v\left(x\left(t, x_{0}\right)\right) \frac{\partial v}{\partial x}\left(x\left(t, x_{0}\right)\right)$, i.e. $v\left(x\left(t, x_{0}\right)\right)$ satisfies the same differential equation as $J$ but with a different initial condition.

\section{EXERCISE 3.1.4.: Let}

$$
\frac{\partial n}{\partial t}+\nabla \cdot(v n)=-\mu n
$$

$\mu\left(t, x_{0}\right)$ a death rate. And let

$$
\zeta\left(t, x_{0}\right) \stackrel{\text { def }}{=} \rho\left(t, x_{0}\right) J\left(t, x_{0}\right),
$$

i.e. if there were no deaths we would have $\zeta\left(t, x_{0}\right)=n\left(0, x_{0}\right)$. Show that

$$
\frac{d \zeta}{d t}=-\mu \zeta \text {. }
$$

Interpret the result in intuitive terms.

EXERCISE 3.1.5: What are the dimensions of $\rho$ and $J$, of $\zeta$ ?

\subsection{The (local) loss of p-mass}

$P$-mass can disappear locally from the interior of $\Omega$ through deaths (or emigration) and through jumping to some other place in $\Omega$. Of course in the latter case the mass returns elsewhere in $\Omega$. On the other hand $p$-mass can disappear through transport across the boundary $\partial \Omega$ of $\Omega$. We shall discuss these two forms of disappearance of $p$-mass separately. In subsection 5.2 the connection will be made again.

\subsubsection{The local disappearance of $p$-mass from the interior of $\Omega$.}

From law of mass-action considerations the local disappearance of $p$-mass at $x$ can always be expressed as 


$$
-\delta_{s-n} n(x)=-\alpha(x) n(x) .
$$

$\alpha$ itself may be a sum of various terms like natural mortality rate, predation intensity, rate of jumping to elsewhere in $\Omega$ etc. Each of these terms can depend in its own way on time or on the state of the p-system as a whole. For example, if $x$ denotes size and $\alpha_{P}$ denotes a predation component of $\alpha$ and $m$ denotes the distribution of predator size $y$, then for randomly searching predators (i.e. predators for which satiation effects for example play no role)

$$
\alpha_{P}(x)=\int_{\Omega_{y}} \beta(x, y) m(y) d y,
$$

where $\beta(x, y) d x$ is the searching intensity for a predator of size $y$ for prey in the size bracket $(x, x+d x)$ (we have to consider a prey size interval here as predators search for prey, not for prey per unit of prey size!). (In part B Daan discusses i.a. the possible functional forms of $\beta$ and the problems of determining it for commercially relevant marine fishes).

The reasoning for jumps goes as follows: let $\gamma(x, y) \Pi d y_{i}$ be the probability per unit of time that an individual at $x$ will jump to $\Pi\left(y_{i}, y_{i}+d y_{i}\right)$ then the contribution $\alpha_{J}$ to $\alpha$ due to this kind of jump is

$$
\alpha_{J}(x)=\int_{\Omega} \gamma(x, y) \Pi d y_{i} .
$$

This can always be rewritten as

$$
\gamma(x, y)=\alpha_{J}(x) p(x, y),
$$

where $p(x, \cdot)$ is the probability density of the "touch down" position for jumps starting at $x$.

REMARK 3.2.1: $\gamma$ and $p$ may contain delta-"functions" with respect to $y$. See section 5 for a discussion centering around the problem of the occurrence of delta-functions in the definition of the $p$-differential generator.

Sometimes we have two or more touch down positions due to the individual splitting into two or more separate individuals. In that case it is still possible to write $\gamma(x, y)=\alpha_{J}(x) p(x, y)$ but the probability density $p(x, \cdot)$ now is a mixture of two or more probability densities, those of the 1st, 2 nd, 3 rd etc. division product. Even more generally the number of division products may be a random variable with distribution $i \mapsto q_{i}(x)$. In that case

$$
p(x, y)=\frac{1}{r(x)} \sum_{i} q_{i}(x) \sum_{j=1}^{i} p_{i j}(x, y)
$$

with

$$
r(x)=\sum_{i} i q_{i}(x)
$$

where $p_{i j}(x, \cdot)$ is the probability density of the touch down position of the $j$ th division product of an individual splitting into $i$ products, and $r(x)$ is the mean number of division products derived from an individual which divided while it was in state $x$.

Quantities like $\alpha_{J}$ and $p(x, y)$ may depend again on time or on the state of the system as a whole. They should be calculated from submodels on the individual level. We shall not go into the various possibilities here, with one exception: the generalization to higher dimensional $i$-state spaces of the "divide on crossing a stochastic threshold" mechanism from I.4.1. Such a generalization will be needed for example if we wish to extend the model from I.4 to include cell age as an $i$-state variable as in example 1.4. from section 1. As another example you may think of a modification of the PPP-problem from example 1.10, in which it is assumed that the amount of resources in as yet unexploited patches is not constant but varies randomly over the patches. A final example comes from environmental toxicology: one of the $i$-state variables is concentration of poison in the individual and death occurs when the poison level passes some random threshold (which is assumed to have been set at birth).

In order to be specific we shall proceed as if crossing the stochastic threshold results in death. Let $\mathscr{F}(t)$ denote the probability that an individual born alive at $t=0$ is still alive at $t$. The probability that it dies between $t$ and $t+d t$, provided it survived till $t$, is precisely what we called $\alpha_{D}(x(t), t) d t$. Therefore $\mathscr{F}$ can be calculated from

$$
\frac{d \mathscr{F}}{d t}=-\alpha_{D}(x(t), t) \mathscr{F}(t), \quad \mathscr{F}(0)=1
$$

or

$$
\mathscr{T}(t)=\exp \left(-\int_{0}^{t} \alpha_{D}(x(\tau), \tau) d \tau\right)
$$


Let $S(x)$ denote the probability that $x$ is below threshold. Then

$$
\mathscr{F}(t)=\mathscr{G}(x(t)) / \mathscr{G}(x(0))
$$

Therefore

$$
\alpha_{D}(x, t)=-\frac{d \log \mathscr{F}(t)}{d t}=-D\left(\log (\mathscr{S}(x)) \frac{d x}{d t}=\sum \mu_{i}(x) v_{i}(x, t)\right.
$$

with

$$
\mu_{i}=-\frac{\partial}{\partial x_{i}} \log (S(x))
$$

(NB. We assume here that the $i$-state movement is such that $S(x(t))$ is guaranteed to decrease with time!)

REMARK 3.2.2: The definition of the $\mu_{i}$ implies that

$$
\frac{\partial \mu_{i}}{\partial x_{j}}=\frac{\partial \mu_{j}}{\partial x_{i}} .
$$

Conversely condition (3.2.7) is also sufficient (provided $\Omega$ is simply connected) for a set of $\mu_{i}$ to define a function $\mathcal{G}$, unique up to a multiplicative constant, through

$$
G(x)=\exp \left(-\int_{x_{0}}^{x} \mu(x) \cdot \frac{d x}{d s} d s\right)
$$

for some path $\Sigma$ joining $x_{0}$ and $x$ and parametrized by s. Formulae (3.2.8) and (3.2.6) are the direct generalization to higher dimensional state spaces of (I.4.1.5) and (I.4.1.6) with (for $\Omega$ 1-dimensional)

$$
\mathcal{G}(x)=1-\int_{0}^{x} \phi_{b}(\xi) d \xi .
$$

(Working with $\mathcal{G}$ instead of $\phi_{b}$ allows us to bypass detailing the relation between a single threshold variable and the $k$ state variables, $k>1$.)

EXERCISE 3.2.3: Write down the local parts of the differential generators of example 1.1 (the invertebrate predator) and 1.10 (the PPP-problem with the amount of resources in newly discovered patches fixed). Call the densities of prey and predator in search of a patch $P$ and $Q$ respectively, and the corresponding rate constants $\zeta$ and $\eta(x)$.

EXERCISE 3.2.4: Write down the local mass loss part of the differential generator of the binary fission model from example 1.4 from section 1 on the assumptions that (i) division rate depends only on size, not on age, (ii) growth may be time dependent, (iii) the same stochastic division threshold model applies as has been introduced in I.4, and (iv) cell death rate is only age dependent. Where does the lost mass reappear?

EXERCISE 3.2.5: In the Asterionella example 1.6 from section 1 assume that breakage of colonies is due to randomly occurring randomly sized shocks that break all bonds older than a certain age. In that case there exists an increasing function $b: \mathbb{R}^{+} \rightarrow \mathbb{R}^{+}$such that $b\left(a_{i}\right) d t$ equals the probability of the breaking of the $i$ th and older bonds, and $\left[b\left(a_{i+1)}-b\left(a_{i}\right)\right] d t\right.$ equals the probability that the $(i+1)$ th and older bonds break but not the $i$ th and any younger bonds. Write down the local mass loss terms of the $p$-differential generator. (Assume that division rate $d$ only depends on cell age and that there is no death.) Where does the lost mass reappear in $\Omega$ ? (NB colonies of size 2 are a bit special as far as reappearance of mass from division is concerned.)

EXERCISE 3.2.6: Write down the local mass loss term of the modified PPP-problem, with the initial amount of resources in a patch a random variable. Hint: Let $\mathcal{K}$ be the survivor function of the initial amount of resources, i.e. $P\left\{r_{0}>\rho\right\}=\mathscr{K}(\rho)$. Use the calculations of exercise 1.12 to express $\subseteq$ in $\mathscr{K}$

\subsubsection{The disappearance of p-mass across the boundary of $\Omega$}

$P$-mass can get lost across the boundary $\partial \Omega$ of $\Omega$ e.g. when animals die, or cells divide, when their $i$-state reaches a fixed barrier. Such a loss of $p$-mass does not show up in the defining formula for calculating the differential generator $A$ but in the definition of its domain. The fact that $A$ is assumed to act on functions on $\Omega$ is part of its definition. In the case that we are dealing with loss of $p$-mass and deterministic flow only, no side conditions are needed. 
Another point is that $p$-mass lost by flowing across the boundary of $\Omega$ may reappear elsewhere. Therefore we have to be able to calculate how much mass flows out of $\Omega$ and where along $\partial \Omega$. As a start we notice that, except for some singularities which may occur when $v$ is no longer smooth at the boundary of $\Omega$ and which shall be dealt with in subsection 3.4, $p$-mass can only leave $\Omega$ when the local speed vector $v$ points out of $\Omega$. The part of the boundary where this is the case we shall call $\partial_{-} \Omega$. To calculate the $i$-state "volume" that flows out of $\Omega$ per unit of time we have to decompose the local flow rate into a component orthogonal to the boundary which contributes to the outflow of $p$ mass, and a component parallel to the boundary which does not contribute. This can be done by means of the outward normal vector $\nu$ (see fig. 3.2.1, or appendix A). The volume that flows across a small unit of area $d \sigma$ of $\partial \Omega$ surrounding $x$ in a small time interval $d t$ equals $v(x) d t \cdot v(x) d \sigma$.

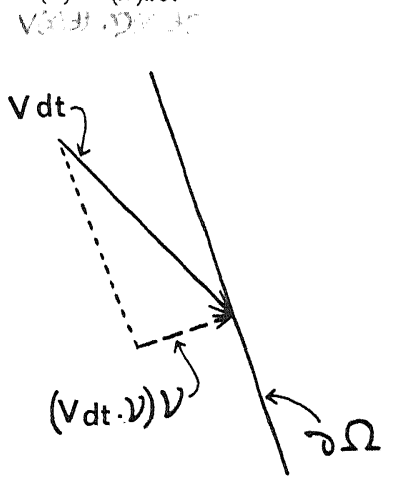

Figure 3.2.1

Therefore the local mass flow rate per unit of area, or, as it is usually called, the flux, out of $\Omega$ equals

[local flux out of $\Omega$ at $x \in \partial_{-} \Omega$ ] $=v(x) \cdot v(x) n(x)$

and

$$
\text { [total mass leaving } \left.\Omega \text { across } \partial_{-} \Omega\right]=\int_{\partial_{-} \Omega} \nu \cdot \nu n d \sigma .
$$

For further calculations it is sometimes helpful to introduce a new quantity, the flux vector $\phi=\left(\phi_{1}, \cdots, \phi_{k}\right)^{T}$. $\phi$ is defined as the amount of mass that flows per unit of time and per unit of area along a point in the positive $x_{1}$ to $x_{k}$ directions. For a deterministic movement through $i$-state space we therefore have

$$
\phi=v n \text {. }
$$

This way we can write the local flux across a $(k-1)$ dimensional surface $\Sigma$ as

$$
\text { [local flux across } \Sigma \text { at } x]=\phi(x) \cdot \nu(x) \text {. }
$$

The importance of this formula is that it still applies when $\phi$ has to be calculated in a different manner, e.g. when there are also stochastic components to the continuous $i$-state movements. It also forms the basis for the Euler derivation of the transport equation set forth in subsection 3.1.2.

EXCERCISE 3.2.7. What is the local rate of mass flow out of the interior of $\Omega$ across boundary segment (0), (see fig. 1.1 ), and therefore the local reappearance rate on the $s$-axis in example 1.1 from section 1 (the invertebrate predator). What is the arrival rate locally at the line $\tau=\tau_{e}$ ? Where does this mass reappear?

EXERCISE 3.2.8: Assume in the PPP-problem with fixed initial resources in a partch (example 1.10) that prey "take the wing" when a patch gets exhausted. What is the total production of prey emigrants per unit of time? Assume that predators leave empty prey patches as well. What is the production rate of predator emigrants?

EXERCISE 3.2.9: Assume that Asterionella colonies break only at the exact moment that the oldest bond reaches age A. What is the local rate of mass loss at this boundary? Where in $\Omega$ (and how) does this mass reappear? (NB colonies of size 1 are a bit special as far as the reappearance of mass is concerned.) 


\subsection{The (re)appearance of p-mass}

New $p$-mass can appear in two manners: locally in the interior of $\Omega$ and at the boundary of $\Omega$. We shall discuss these two possibilities separately. In subsection 5.2 the connection will be made again.

\subsubsection{The (re)appearance of $p$-mass in the interior of $\Omega$}

If per unit of time an amount of $p$-mass $b \Pi d x_{i}$ is added to a volume element $\Pi\left(x_{i}, x_{i}+d x_{i}\right)$ this results in a component

$$
\delta_{s+n}=b
$$

in the defining formula for the $p$-differential generator $\mathrm{A}$. There remains the question of how $b$ can be calculated. In principle $b$ is the sum of two terms: the results of new births and of jumps of the $i$-state process. Of course, if an individual divides into two or more parts it depends on our conventions with respect to the concept of "parent" whether we speak of a jump and birth(s) or of births only. To simplify the presentation we shall call every "touch down" in $\Omega$ a birth, independent of whether this is a real birth or just a reappearance after a jump.

To calculate $b$ we split up the various births with respect to their origin, calculate the corresponding birth rates through a law of mass action argument and cumulate over origins. That is, if the separate components of $\Omega$ are denoted as $\Omega_{i}$,

$$
b(x)=\sum_{i}\left\{\int_{\Omega} r(y) p(y, x) \alpha_{J}(y) n(y) d y+\int_{\partial-\Omega_{i}} r(y) p(y, x) \phi(y) \cdot v(y) d \sigma\right\},
$$

(assuming that one can only be born out of individuals of the same population and that there is no immigration), where $r, p$ and $\alpha_{J}$ in the first integral have been defined in (3.2.3) to (3.2.5), $r$ and $p$ in the second integral have the same interpretation as in the first integral, $\phi$ and $\nu$ and $d \sigma$ have been introduced in subsection 3.2.2 (a more extended discussion of surface integrals may be found in appendix $\mathrm{A}$ ).

There is still one snag in calculating the various terms in (3.3.2) which has to do with the way in which $p$ is calculated. We shall show the problems involved by means of an example.

EXAMPLE 3.3.1: Cell division into unequal parts. Consider a cell division model in which the size of the two daughter cells are two random fractions, $\theta$ and $1-\theta$, of the parents size. Let $f$ denote the probability density of $\theta$ (with $f(\theta)=f(1-\theta)$ so that $f(\theta)+f(1-\theta)=2 f(\theta))$, Then, if $\alpha_{d}$ denotes the division rate as a function of size,

$$
b(x)=\int_{0}^{1} 2 f(\theta) \theta^{-1} \alpha_{d}\left(\frac{x}{\theta}\right) n\left(\frac{x}{\theta}\right) d \theta .
$$

The reason for the factor $\theta^{-1}$ is that for a fixed value of $\theta$ births in the range $(x, x+d x)$ come from parents in the range $\left(\frac{x}{\theta}, \frac{x}{\theta}+\frac{d x}{\theta}\right)$ and we have to discount a compression factor $\theta$ of the interval during the jump. More formally, if $p(y, x) d x$ equals the probability of touchdown in $(x, x+d x)$ of a randomly chosen division product of a parent cell of size $y$, like in (3.2.5), then (using integration rule (ii) from appendix A with $\theta=\frac{x}{y}$ )

$$
\int_{\theta_{1}}^{\theta_{2}} f(\theta) d \theta=\int_{x_{1}}^{x_{2}=\theta_{2} y} p(y, x) d x=\int_{x_{1}}^{x_{2}} f\left(\frac{x}{y}\right) y^{-1} d x .
$$

So we find

$$
p(y, x)=f\left(\frac{x}{y}\right) y^{-1} .
$$

According to (3.3.2) we have to integrate $2 \alpha_{d}(y) p(y, x) n(y)$ with respect to $y$ to get $b(x)$. This way we find again (applying integration rule (ii) with $y=\frac{x}{\theta}$ )

$$
\int_{\Omega} 2 p(y, x) \alpha_{d}(y) n(y) d y=\int_{1}^{0} 2 f(\theta) \alpha_{d}\left(\frac{x}{\theta}\right) n\left(\frac{x}{\theta}\right) \frac{\theta}{x} \frac{d}{d \theta}\left(\frac{x}{\theta}\right) d \theta=\int_{0}^{1} 2 f(\theta) \alpha_{d}\left(\frac{x}{\theta}\right) n\left(\frac{x}{\theta}\right) \theta^{-1} d \theta
$$

as in (3.3.3.).

Numbers are conserved, not densities. Therefore we have to be very careful in ascertaining how the volume elements at the places of take off and touch down are connected, as the various volume conversion factors enter into the calculation in a nontrivial way. An analogous remark applies to the connection of surface and volume elements for 
individuals taking off across $\partial_{-} \Omega$. An example of the latter type of calculation will be shown in the next subsection where the instream of $p$-mass across $\partial \Omega$ is considered.

EXERCISE 3.3.2: Calculate the local birth terms for the Asterionella models from exercises 3.2.5 and 3.2.9.

\subsubsection{The (re) appearance of p-mass across the boundary of $\Omega$}

$P$-mass can only stream into $\Omega$ across $\partial \Omega$ at those points where $v$ is directed inwards, or more formally, where $v . \nu$ is negative. We shall call that part of the boundary $\partial_{+} \Omega$. On $\partial_{+} \Omega$ the arrival rate per unit of surface area $b_{\partial}$ should equal the inward flux at the boundary, giving rise to the boundary condition

$$
-v(x) \cdot \phi(x)=-v(x) \cdot v(x) n(x)=b_{\partial}(x)
$$

There remains the problem of calculating $b_{\partial}(x)$. This can be done along the same lines as the calculation of the interior local arrival rate (3.3.2). Only now $p_{\partial}$ has the dimension (surface area) ${ }^{-1}$ instead of (volume) ${ }^{-1}$, and we have to keep careful track how volume or surface elements at take off transform into surface elements at touch down. We shall but give an example.

EXAMPLE 3.3.3: The invertebrate functional response continued. In the excercises (3.1.2, 3.2.3 and 3.2.7) we have already encountered various parts of the differential generators for the invertebrate predator of example 1.1. The full $p$ differential generator is displayed in subsection 4.1. Here we shall concentrate on $\partial_{+} \Omega_{2} \partial_{+} \Omega$ consists of two parts. The maximum satiation of searching predators $s_{m}$ bounding $\Omega_{0} \subset \mathbb{R}$ from above and the boundary segment (2) bounding $\Omega_{1} \subset \mathbb{R}^{2}$ to the right. (see figure 1.1). At $s_{m}$ we just have to make sure that there is no instream of maximally satiated searching predators by setting

$$
\phi\left(s_{m}\right)=-f\left(s_{m}\right) n_{0}\left(s_{m}\right)=0,
$$

(where $f$ was the digestion rate), or equivalently

$$
n_{0}\left(s_{m}\right)=0 \text {. }
$$

At boundary segment (2) there is an instream of predators having just started search. The predators arriving at $\left(s, \tau_{m}(s)\right)$ come from $(s, 0)$. Predators leave there at a rate $x g_{0}(s) n_{0}(s)$. However, we need the arrival rate per unit of boundary length. A line element of length $d s$ on the $s$-axis connects with a line element of length $d \sigma=\left((d s)^{2}+\left(\frac{d \tau_{m}}{d s} d s\right)^{2}\right)^{\frac{1}{2}}=\left(1+\left(\frac{d \tau_{m}}{d s}\right)^{2}\right)^{\frac{1}{2}} d s$. (Compare formula (A2.6); we parametrize the touch down boundary with the position at take off and match number of predators leaving per unit of time, an integral over a segment of the $s$-axis, with number of predators arriving, an integral over a corresponding segment of the touch down boundary.) The arrival rate per unit of length equals

$$
b_{\partial}\left(s, \tau_{m}(s)\right)=x g_{0}(s) n(s)\left(1+\left(\frac{d \tau_{m}(s)}{d s}\right)^{2}\right)^{-\frac{1}{2}}
$$

This should equal

$$
\phi\left(s, \tau_{m}(s)\right) \cdot-\nu\left(s, \tau_{m}(s)\right)=n_{1}(s)\left[\begin{array}{l}
1 \\
-a s
\end{array}\right] \cdot\left[\begin{array}{l}
1 \\
\frac{d \tau_{m}}{d s}(s)
\end{array}\right]\left(1+\left(\frac{d \tau_{m}(s)}{d s}\right)^{2}\right)^{-\frac{1}{2}} .
$$

Therefore on boundary segment (2) we should have the side condition

$$
\left(1-a s \frac{d \tau_{m}}{d s}(s)\right) n_{1}\left(s, \tau_{m}(s)\right)=x g_{0}(s) n_{0}(s)
$$

EXERCISE 3.3.4: Derive appropriate side conditions for example 1.4 (binary fission), 1.6 (Asterionella) and 1.10 (the PPP-problem) of section 1 . In the Asterionella example consider both the cases covered by the assumptions made about the breakage mechanism in exercise 3.2.5 and in exercise 3.2.9.

\subsection{Boundaries and side conditions: picking up the strands}

In subsection 3.2 .2 we encountered the boundary $\partial_{-} \Omega$ defined by $\nu \cdot \nu>0$, and in subsection 3.3 .2 the boundary $\partial_{+} \Omega$ 
defined by $\nu \cdot \nu<0$. The definition of the differential generator $\mathrm{A}$ will have to include side conditions on $\partial_{+} \Omega$ for otherwise A will certainly not define a unique semigroup: if we do not prescribe what is to be put onto a conveyor belt at its start we cannot foretell its contents. On $\partial_{-} \Omega$ no side conditions are needed. In fact if we would prescribe side conditions on $\partial_{-} \Omega$ there would probably no longer exist a semigroup associated with the would be differential generator so defined: once we have prescribed what happens at the start of as well as on a conveyor belt we are no longer free to prescribe what is going to leave it at its end.

Apart from $\partial_{-} \Omega$ and $\partial_{+} \Omega$ we may have one piece of boundary left: $\partial_{0} \Omega$ for which $v \cdot \nu=0$. If the vector field $v$ is smooth on $\bar{\Omega}$ (the closure of $\Omega$ ) then it is impossible for individual trajectories to reach or leave $\partial_{0} \Omega$ so no boundary condition has to be prescribed nor should it be. The following example shows that this need not hold if our smoothness assumption is relaxed.

EXAMPLe 3.4.1: Von Bertalanffy growth starting from zero initial size. In the model for size dependent population growth in ectotherms from I.3 we encountered the equation

$$
\frac{\partial n}{\partial t}=-\frac{\partial g_{1} n}{\partial l}-\mu n
$$

with

$$
g_{1}(l)=(3 \eta)^{-1}(\kappa \nu f-\zeta l)^{+}
$$

and

$$
\phi\left(l_{b}\right)=g_{1}\left(l_{b}\right) n\left(l_{b}\right)=b
$$

with

$$
b=\int_{\Omega} \lambda_{1}(l) n(l) d l
$$

Biological considerations imply that birth length $l_{b}$ should be positive. However, from a mathematical point of view nothing keeps us from considering the limiting case $l_{b}=0$. (In fact, this can be a useful step in dealing with models in which evolution drives the population towards smaller and smaller $l_{b}$ ).

So far there is no problem. $g_{1}(0)>0$ and (3.2.1c) provides the necessary boundary condition. But now consider the same model but with weight as the $i$-state. Derived from first principles the $p$-equation now looks like

$$
\begin{aligned}
& \frac{\partial n}{\partial t}=-\frac{\partial g_{3} n}{\partial w}-\mu n \\
& g_{3}(w)=\eta^{-1}\left(\kappa \nu f w^{2 / 3}-\zeta w\right)^{+} \\
& \phi\left(w_{b}\right)=g_{3}\left(w_{b}\right) n\left(w_{b}\right)=b \\
& b=\int_{\Omega} \lambda_{3}(w) n(w) d w
\end{aligned}
$$

In the limiting case for $w_{b} \downarrow 0$ the left hand boundary at $w_{b}$ belongs to $\partial_{0} \Omega$. Still, since (3.4.1) and (3.4.2) are equivalent, (3.4.2) should be a genuine model also for $w_{b}=0$, provided that the boundary condition (3.4.2c) is replaced by

$$
\lim _{w \downarrow 0} \phi(w)=\lim _{w \downarrow 0} g_{3}(w) n(w)=b
$$

What happens in this example is that indeed for genuine weight distributions, i.e. functions $n: \Omega \rightarrow \mathbb{R}^{+}$such that

$$
\int_{\Omega} n d w<\infty
$$

3.4.2c') is a perfectly valid boundary condition as it is equivalent to

$$
n(w)=\frac{\eta b}{k \nu w^{2 / 3}}+o\left(w^{-2 / 3}\right) \text { for } w \downarrow 0
$$

which is compatible with (3.4.3). However, would $g_{3}(w)$ have gone to zero smoothly for $w \downarrow 0$ then an expression like (3.4.2.c') could never be fulfilled by a function satisfying (3.4.3).

On the individual level the anomaly has to do with the fact that for the initial condition $w(0)=0$ the von Bertalanffy growth equation $d w / d a=g_{3}(w)$ does not have a unique solution: a solution may either stay zero for ever (as we expect from $g_{3}(0)=0$ ) or may leave zero at any nonnegative time. It is this possibility of growing away from $0=\partial_{0} \Omega$ that makes that we have to prescribe a boundary condition at $\partial_{0} \Omega$. For growth laws which are smooth also in 0 like $g_{1}$ no such anomalous behaviour is possible: either 0 belongs to $\partial_{+} \Omega$ and we have to prescribe a boundary 
condition, or it belongs to $\partial_{0} \Omega$ or $\partial_{-}^{*} \Omega$ and we should not do so. ( Of course there remains the biological anomaly that $g_{1}(0)>0$, making individuals grow away from zero size. The explanation here is that $g_{3}$ and $g_{1}$ were based on the assumption that individuals of all sizes are structuraly similar, which can only apply from a sufficient size onwards.)

In the same manner as we may have inflow of mass across a subset $\partial_{0} \Omega$ of $\partial_{0} \Omega$, we may have outflow across a subset $\partial_{0} \Omega \subset \partial_{0} \Omega$, but biological examples do not seem to abound.

If we have taken care of prescribing the right side conditions on $\partial_{+} \Omega$ and $\partial_{0+} \Omega$, then it is generally possible, in the linear case at least, to prove existence and uniqueness of solutions of our $p$-differential equation and therefore the existence and the uniqueness of an associated semigroup of next $p$-state transformations, by means of a generation expansion, i.e. a series expansion in which the subsequent terms are just the $i$-state densities of individuals in subsequent biological "generations" (where generation should be interpreted in a generalized sense in that $i$-state jumps are treated as combined death-birth events). Therefore the side conditions which have passed in revue are the only ones that are needed.

REMARK 3.4.2: It is not uncommon for $\partial \Omega$ to consist of a finite number of smooth surfaces connected by edges (see e.g. figure 1.1). In an edgepoint there does not exist a well defined normal $\nu$. Usually it is clear from the interpretation to which of its adjoining surfaces we should assign such a point and therefore what we should choose for $\nu$ in our calculations. However, in general we can just leave unspecified what happens as the edges only form a subset of $\partial \Omega$ of measure zero.

EXERCISE 3.4.3: Discuss the possibilities of calculating an optimal birth weight under $r$-selection in our Daphnia model from I.3. Here $r$-selection refers to selection for the maximum possible asymptotic population growth rate $r$ as introduced in 1.3.3. Assume an energetic trade off between birth rate and birth weight as expressed by the factor $\left(\omega w_{b}\right)^{-1}$ in formula (I.3.1.4).

\subsection{Summary and concluding remarks}

We now have come at the end of the model building sections. In the following sections we shall discuss how our basic p-equations may be used to derive other types of equations by means of various transformation rules and/or limiting arguments. However, before going on it may be useful once more to put the variety of arguments that have passed in revue into perspective.

The specification of a structured population model embraces

- the choice of an individual state space $\Omega$, a domain in $\mathbb{R}^{k}$ (or the union of a number of such domains, possibly for different $k$ )

-a recipe for calculating the velocity $v$

-a recipe for the calculation of the per capita disappearance rate $\alpha$

-a recipe for the calculation of the (re)appearance rates $b$ and $b_{\partial}$.

In subsections 2.2 and 2.3 we have discussed respectively the conceptual intricacies of constructing models for individual behaviour and the coupling of individuals into populations. Together these two subsections covered all four ingredients mentioned above, albeit in a rather abstract, philosophical fashion. The examples from section 1 serve to add some concreteness. In the present section we discussed how the abstract considerations from section 2 can be translated into our main working tool: p-equations. In 3.1, 3.2.1 and 3.3.1 we discussed the bits and pieces corresponding respectively to $v, \alpha$ and $b$, of the defining recipe of the $p$-differential generator, telling how it acts on the elements of its domain. In subsections 3.2 .2 and 3.3.2 and 3.4 we discussed the other necessary component of its definition, the description of its domain, with special reference to respectively $\Omega$ and $b_{\partial}$. In 3.1 and 3.2 .2 we dealt with the process of deterministic change: in 3.1 we considered the continuous shifting of the $i$-state distribution in the interior of $\Omega$ resulting from $v$, in 3.2.2 we saw how this shifting may lead to deaths or $i$-state jumps by individuals being pushed inexorably over the boundary $\partial_{-} \Omega$. In 3.2 .1 and 3.3 we considered chance in the form of random deaths, births and $i$-state jumps: in 3.2.1 deaths and take-offs represented by $\alpha$, in 3.3 births and touch downs represented by $b$ and $b_{\partial}$.

There is still one possible mode of $i$-state behaviour left which we refrained from discussing: stochastic continuous $i$-state movements. These are covered in appendix $\mathrm{B}$.

EXERCISE 3.5.1 The stochastic threshold models from 3.2.1 hold a kind of intermediate position with respect to the 
change versus chance dichotomy: in these models we can make the occurrence of deaths or $i$-state jumps deterministic by shoving in an extra $i$-state variable representing the threshold, but at the price of a higher dimensional $i$-state space and additional randomness in the state at birth. Rephrase the "division on reaching a stochastic threshold" model from I.4 in the manner indicated. Also integrate the resulting $p$-equation over the threshold variable to rederive the $p$ equation from 1.4 .

\section{Integration along characteristics, transformation of variables, and the following of cohorts through time.}

In our treatment of size dependent reproduction in ectothermic animals in I.3 we achieved for the case of constant food density a transformation into an equivalent renewal equation through a technique called integration along characteristics. In this section we shall consider this technique and various related techniques for transforming $p$ equations from a more general perspective. Of course the usefulness of these techniques in any specific instance is contingent upon the resulting equation being relatively more tractable than the original one. Whether this is the case depends on the presence of some exploitable special properties which are brought to the fore by the transformation.

In the following we shall proceed as if $\Omega$ consists of only one $k$-dimensional component. If $\Omega$ has more than one component then each component can be treated separately along the lines discussed.

\subsection{Integration along characteristics}

Characteristics are curves in $(k+1)$-space $\Omega \times \mathbb{R}^{+}$, spanned by the $i$-state coordinates and time, along which the material derivative

$$
\frac{D n}{D t}=\frac{\partial n}{\partial t}+\nabla n \cdot v
$$

reduces to an ordinary (time) derivative. These curves correspond to the trajectories of individuals conditional on the non-occurrence of $i$-state jumps. Sometimes we shall be sloppy and identify these curves with their projections on $\Omega$ which will then be called characteristics as well. When the time dependence of the individual speed $v$ through $\Omega$ is explicitly given and the same applies to the relative jump/death rate $\alpha$, the birth/reappearance rate $b$ and the (re)appearance rate $b_{\partial}$ of $p$-mass at $\partial_{+} \Omega$, then it is possible to obtain explicit expressions for $n$ along these curves. If instead one or more of the quantities $v, \alpha, b$ and $b_{\partial}$ are generated by the model we can still proceed as if they were known. The result is a formula expressing $n$ in terms of some as yet unknown functions. This expression for $n$ is equivalent to the partial differential equation from which it was derived. If we substitute it in the remaining part of the $p$-equation we end up with an equation of a different type containing the same information. Obviously we may only expect this new equation to be more manageable if sufficiently many of the quantities $\nu, \alpha, b$ and $b_{\partial}$ are specified explicitly. The calculations from I.3.4 provide a good example.

REMARK 4.1.1.: In the general theory of partial differential equations one sometimes refers to the characteristics as curves in $(k+2)$-space, spanned by the $i$-state coordinates, time and $n$. Our characteristics are projections of these curves on $(k+1)$-space.

Let $\theta$ be a parameter which is equivalent to time, except for a temporary arbitrariness of its origin, then the characteristics are defined by

$$
\frac{d t}{d \theta}=1, \quad \frac{d x}{d \theta}=v(t, x)
$$

the $p$-equation is given by

$$
\frac{\partial n}{\partial t}+\nabla n \cdot v=-n \nabla \cdot v-\alpha n+b
$$

then along the characteristics

$$
\frac{d n}{d \theta}=\frac{\partial n}{\partial t} \frac{d t}{d \theta}+\nabla n \cdot \frac{d x}{d \theta}=\frac{\partial n}{\partial t}+\nabla n \cdot v
$$

and therefore

$$
\frac{d n}{d \theta}=-n(\nabla \cdot \nu+\alpha)+b
$$


This equation is linear, and allows the explicit solution

$$
n(\theta)=n(0) \exp \left(-\int_{0}^{\theta}(\nabla \cdot v+\alpha) d \theta_{1}\right)+\int_{0}^{\theta} b \exp \left(-\int_{\theta_{2}}^{\theta}(\nabla \cdot v+\alpha) d \theta_{1}\right) d \theta_{2} .
$$

If for example we choose points of $\partial_{+} \Omega$ as initial conditions for (4.1.1) we can calculate $n(0)$ from the corresponding side condition. The dilation factor $\exp \left(-\int_{0}^{\theta} \nabla \cdot v d \theta_{1}\right)$ describes the result of the contraction or expansion of the conveyor belt in the course of its movement, the factor $\exp \left(-\int_{0}^{\theta} \alpha d \theta_{1}\right)$ describes the cumulated local relative loss of $p$-mass through jumps or deaths and the term after the plus sign consists of the cumulation of the various additions to the $p$ mass after accounting for the changes undergone during transport.

Of course points on $\partial_{+} \Omega$ provide only one possible starting point for the integration of (4.1.2) and (4.1.3). The calculations in II.10 for the cell proliferation model from I.4 (see exercise 4.1 .5 below) show for example that it can also pay to consider starting points inside $\Omega$.

In I.3.4 we substituted the result from our integration along characteristics into the side conditions to arrive at a renewal equation. The end result of the calculations in II.10 also was a renewal equation. The example below shows that renewal equations are by no means the only possible end result. (In chapter IV we shall discuss in detail the $i$ features that allow $p$-behaviour to be captured by a renewal equation.)

EXAMPLE 4.1.2: The invertebrate functional response. The p-equation for example 1.1 from section 1 is, in obvious notation

$$
\begin{aligned}
& \frac{\partial n_{0}(t, s)}{\partial t}=\frac{-\partial f_{0}(s) n_{0}(t, s)}{\partial s}-x g_{0}(s) n_{0}(t, s)+ \\
& \text { digestion } \quad \text { starting pursuit } \\
& +\int_{\tau_{0}}^{\tau_{m}(s)} \mu n_{1}(t, s, \tau) d \tau+(1-q) n_{1}\left(t, s, \tau_{e}+\right)+n_{1}(t, s, 0+)
\end{aligned}
$$

from abortive pursuits from failed strikes from finished meals

$$
\begin{aligned}
& \frac{\partial n_{1}(t, s, \tau)}{\partial t}=\frac{-\partial f_{1}(s, \tau) n_{1}(t, s, \tau)}{\partial s}+\frac{\partial n_{1}}{\partial \tau}(t, s, \tau)-\lambda(\tau) n_{1}(t, s, \tau) \\
& \text { digestion/ingestion proceeding of time escaping prey }
\end{aligned}
$$

with the "transition condition" *

$$
n_{1}\left(t, s, \tau_{e}-\right)=q n_{1}\left(t, s, \tau_{e}+\right) \quad \text { [from successful strikes] }
$$

and the boundary conditions

$$
\begin{aligned}
& n_{0}\left(t, s_{m}\right)=0, \\
& \left(1-a s \frac{d \tau_{m}(s)}{d s}\right) n_{1}\left(t, s, \tau_{m}(s)\right),=x g_{0}(s) n_{0}(t, s) \text { [from started pursuits], }
\end{aligned}
$$

where

$$
\begin{aligned}
& f_{0}(s)=-a s, \quad s_{m}=e^{-a \tau_{m}(c)} c+\left(1-e^{-a \tau_{e}}\right) u / a, \\
& f_{1}(s)=\left\{\begin{array}{c}
-a s+u \\
-a s
\end{array}, \lambda(\tau)=\left\{\begin{array} { l } 
{ 0 } \\
{ \mu }
\end{array} \text { for } \left\{\begin{array}{l}
\tau<\tau_{e} \\
\tau>\tau_{e}
\end{array}\right.\right.\right.
\end{aligned}
$$

and

$$
\tau_{m}(s)=\tau_{e}+\tau_{p}(s),
$$

$\tau_{e}$ the total meal duration, $\tau_{p}$ the duration of a succesful pursuit, and $c$ the search threshold, i.e. $g_{0}(s)=0 \Leftrightarrow s \geqslant c$. These are two partial differential equations, in $t$ and one and two $i$-state variables. However, the second equation, that - If $f: \mathbb{R} \rightarrow \mathbb{R}$, then $f(x+):=\lim _{y \downarrow x} f(y)$ and $f(x-):=\lim _{y \uparrow x} f(y)$, provided these limits exist. 
for $n_{1}$, can be eliminated by integrating it along characteristics, leaving us with one partial differential equation with delayed arguments. As we are especially interested in the calculation of equilibria (remember I.2), and since at equilibrium time delays drop out, this presents a considerable simplification.

The characteristics of $(4.1 .5 \mathrm{~b})$ are given by

$$
\begin{aligned}
t & =t_{0}+\theta, \\
\tau & =\tau_{0}-\theta=\tau_{m}\left(s_{0}\right)-\theta=\tau_{e}+\tau_{p}\left(s_{0}\right)-\theta, \\
s & =e^{-a \theta} s_{0}+(u / a)\left[1-e^{-a\left(\theta-\tau_{p}\left(s_{0}\right)\right)^{+}}\right] .
\end{aligned}
$$

with $s_{0}$ and $\tau_{0}$ the intersection of the characteristic with $\partial_{+} \Omega_{1}((2)$ in figure 1.1). Given a value of $(s, \tau)$ we can solve (4.1.6b) and (4.1.6c) for $s_{0}$ and $\theta$. Using these values we can express $\left(s_{0}, \tau_{0}\right)$ in $(s, \tau)$. For $s_{0}$

$$
\begin{aligned}
& \tau>\tau_{e}: \log \left(s_{0}\right)-\tau_{p}\left(s_{0}\right)=\tau_{e}-a \tau+\log (s), \\
& \tau<\tau_{e}: \log \left(s_{0}\right)-\tau_{p}\left(s_{0}\right)=\tau_{e}-a \tau+\log \left\{s-(u / a)\left[1-e^{-a\left(\tau_{e}-\tau\right)}\right]\right\} .
\end{aligned}
$$

Our assumption that $\tau_{p}$ is a decreasing function of $s$ guarantees that these equations have a unique solution. For later use we introduce the notation

$$
s_{1}(s):=s_{0}\left(s, \tau_{e}\right), \quad s_{2}(s):=s_{0}(s, 0), \quad \tau_{i}:=\tau_{m}\left(s_{i}\right) .
$$

(Note that for the parameter values of a realistic predator $s_{2}(s)<s$, whereas $s_{1}(s)>s$, and $s_{0}(s, \tau)>s$ for $\tau>\tau_{e}$.) For $n_{1}$ we find

$$
\begin{aligned}
& \tau>\tau_{e}: n_{1}(t, s, \tau)=x g_{0}\left(s_{0}\right) n_{0}\left(t-\tau_{0}+\tau, s_{0}\right)\left(1-a s_{0} \frac{d \tau_{m}\left(s_{0}\right)}{d s_{0}}\right)^{-1} e^{-(a-\mu)\left(\tau_{0}-\tau\right)} \\
& \tau<\tau_{e}: n_{1}(t, s, \tau)=x g_{0}\left(s_{0}\right) n_{0}\left(t-\tau_{0}+\tau, s_{0}\right)\left(1-a s_{0} \frac{d \tau_{m}\left(s_{0}\right)}{d s_{0}}\right)^{-1} e^{a\left(\tau_{0}-\tau\right)-\mu\left(\tau_{0}-\tau_{e}\right)}(1-q) .
\end{aligned}
$$

Substituting this in (4.1.5a) finally gives

$$
\begin{aligned}
& \frac{\partial n_{0}(t, s)}{\partial t}=\frac{\partial a s n_{0}(t, s)}{\partial s}-x g_{0}(s) n_{0}(t, s)+ \\
& \int_{\tau_{e}}^{\tau_{m}(s)} \mu x g_{0}\left(s_{0}(s, \tau)\right) n_{0}\left(t-\tau_{0}(s, \tau)+\tau, s_{0}(s, \tau)\right)\left(1-a s_{0}(s, \tau) \frac{d \tau_{m}\left(s_{0}(s, \tau)\right)}{d s_{0}}\right)^{-1} e^{-(a-\mu)\left(\tau_{0}(s, \tau)-\tau\right)} d \tau+ \\
& (1-q) x g_{0}\left(s_{1}(s)\right) n_{0}\left(t-\tau_{1}(s)+\tau_{e}, s_{1}(s)\right)\left(1-a s_{1}(s) \frac{d \tau_{m}\left(s_{1}(s)\right)}{d s_{1}}\right)^{-1} e^{(a-\mu)\left(\tau_{1}(s)-\tau_{e}\right)}+ \\
& q x g_{0}\left(s_{2}(s)\right) n_{0}\left(t-\tau_{2}(s), s_{2}(s)\right)\left(1-a s_{2}(s) \frac{d \tau_{m}\left(s_{2}(s)\right)}{d s_{2}}\right)^{-1} e^{(a-\mu) \tau_{2}(s)-\mu \tau_{e}}, \\
& n_{0}\left(t, s_{m}\right)=0
\end{aligned}
$$

which is the sought result.

Setting $\frac{\partial \hat{n}_{0}}{\partial t}=0$ gives us an equation for the equilibrium $\hat{n}_{0}$. This has to be combined with the normalization condition

$$
\int_{\Omega_{0}} \hat{n}_{0} d s+\int_{\Omega_{1}} \hat{n}_{1} d s d \tau=1
$$

Given $\hat{n}_{0}$ we can calculate $\hat{n}_{1}$, and from this the functional response $F$ as

$$
F(x)=\int q \hat{n}_{1}\left(s, \tau_{e}+\right) d s .
$$

An easy way to calculate the integrals will be derived in example 4.2.4.

EXERCISE 4.1.3: Write the equation (4.1.9a) for $\hat{n}_{0}$ out in full.

EXERCISE 4.1.4: Calculate the characteristics of the PPP-model from example 1.10. Also calculate the densities $n_{1}$ and $n_{2}$ on $\Omega_{x}$ and $\Omega_{x y}$ resp. on the assumption that the time course of $n_{0}$, the number of empty patches, and of the numbers of the free searching, patchless prey and predators are known.

EXERCISE 4.1.5: Under constant nutrient conditions the reproduction by binary fission model from I.4 can be 
transformed into a renewal equation. Assume to this end that the division rate $b$ is positive only on $(a, 1)$ with $a>\frac{1}{2}$. Integrate the $p$-equation along its characteristic from $a$ to 1 , assuming $n(a)$ to be known. Next do the same on $\left(\frac{1}{2} a, a\right)$, using the boundary condition $n\left(\frac{1}{2} a\right)=0$ and the known $n$ on $\left(\frac{1}{2} a, a\right)$. Use the results to derive a renewal equation for $n(t, a)$. (The answer to this exercise can be found in II.10.)

\subsection{Transformation of variables}

Integration along characteristics provides one way of transforming our p-equations. Another way is provided by a change in $i$-state variables. An example of two $p$-equations which are thus connected can be found in excample 3.4.1, Von Bertalanffy growth starting from zero initial size. Below we shall first discuss the reparametrization of the instate in general. At the end of this subsection the two ideas of characteristic and $i$-state reparametrization will be comm bined.

Let $y$ be the new $i$-state parametrization,

$$
y=h(t, x), x=g(t, y)
$$

where $h$ and $g$ are each others inverses with respect to their second argument, i.e. for all $x, y \in \mathbb{R}^{k}$ and all $b \geq 0$

$$
x=g(t, h(t, x)), y=h(t, g(t, y))
$$

and let $m$ be the corresponding $p$-state, then (by integration rule (ii) from appendix A)

$$
m(t, y)=n(t, g(t, y)) \operatorname{det}\left|D_{y} g(t, y)\right| .
$$

Now let $n$ satisfy

$$
\frac{\partial n(t, x)}{\partial t}+\nabla_{x} \cdot(v(t, x) n(t, x))=-\alpha(t, x) n(t, x)+b(t, x)
$$

For $m$ we have the corresponding equation

$$
\frac{\partial m(t, y)}{\partial t}+\nabla_{y} \cdot(w(t, y) m(t, y))=-\beta(t, y) m(t, y)+c(t, y)
$$

The question is: what is the relationship between the various terms in these two equations?

We start with $v$ and $w$. On the individual level we have

$$
\begin{aligned}
w(t, y) & =\frac{d y}{d t}=\frac{d}{d t} h(t, x)=D_{x} h(t, x) \frac{d x}{d t}+D_{t} h(t, x) \\
& =D_{x} h(t, g(t, y)) v(t, g(t, y))+D_{t} h(t, g(t, y)),
\end{aligned}
$$

or equivalently

$$
=\left[D_{y} g(t, y)\right]^{-1}\left[v(t, g(t, y))-D_{t} g(t, y)\right]
$$

Here we used

$$
0=D_{t} h(t, g(t, y))+D_{x} h(t, g(t, y)) D_{t} g(t, y),
$$

(note that the differentiation for $t$ in the first term only applies to the argument of $h$ !) and

$$
I=D_{x} h(t, g(t, y)) D_{y} g(t, y),
$$

obtained from differentiating (4.2.2) for $t$ and $y$ respectively.

To find the transformation for $\alpha$ and $b$ we use dimensional considerations. The dimensions of $\alpha$ and $\beta$ are both time $^{-1}$. Therefore

$$
\beta(t, y)=\alpha(t, g(t, y)) \text {. }
$$

The dimension of $b$ is (number of individuals) / ( $i$-state volume $\times$ time). The factor transforming $i$-state volumes was $\left|\operatorname{det} D_{y} g(t, y)\right|^{-1}$ (compare (4.2.3a)). Therefore

$$
c(t, y)=b(t, g(t, y))\left|\operatorname{det} D_{y} g(t, y)\right|
$$

(Obviously, if $b$ has to be calculated from some integral over $x$, we also have to make the appropriate changes to 
transform this into an integral over $y$.) Boundary conditions transform in an analogous way: To calculate $c_{\partial}$ we have to multiply $b_{\partial}$ with a factor expressing how a length element of $\partial_{+} \Omega_{x}$ relates to a length element of $\partial_{+} \Omega_{y}$.

* INTERLude 4.2.1: An algebraic check of the transformation rules.

We start with the case of time independent $g$ and $h$. Multiplying (4.2.3b) with $\left|\operatorname{det} D_{x} h\right|=\left|\operatorname{det} D_{y} g\right|^{-1}$ gives

$$
\frac{\partial n}{\partial t}+\left|\operatorname{det} D_{x} h\right| \nabla_{y} \cdot(w m)=-\alpha n+b
$$

So all we have to show is that

$$
\nabla_{x} \cdot(v n)=\left|\operatorname{det} D_{x} h\right| \nabla_{y} \cdot(w m) .
$$

To simplify the notation we shall assume from now on that $\operatorname{det} D_{x} h>0$; the case $\operatorname{det} D_{x} h<0$ only necessitates some selfevident changes in the calculations below. We start by observing that $n(x)=m(h(x)) \operatorname{det} D_{x} h(x)$. Therefore, by the chain rule

$$
\nabla_{x} \cdot(v n)=\sum_{i} \frac{\partial}{\partial x_{i}}\left(v_{i}(x) n(x)\right)=\sum_{i, j} \frac{\partial}{\partial y_{j}}\left[v_{i}(g(y)) m(y) \operatorname{det} D_{x} h(g(y))\right] \frac{\partial h_{j}}{\partial x_{i}} .
$$

This should be equal to

$$
\operatorname{det} D_{x} h \sum_{i, j} \frac{\partial}{\partial y_{j}}\left[\left(D_{x} h(g(y))_{j i} v_{i}(g(y)) m(y)\right] .\right.
$$

To show that this is the case it is sufficient to show that

$$
\sum_{j} \frac{\partial}{\partial y_{j}}\left(\operatorname{det} D_{x} h(g(y)) \frac{\partial h_{j}}{\partial x_{i}}=\operatorname{det} D_{x} h \sum_{j} \frac{\partial}{\partial y_{j}}\left(D_{x} h(g(y))\right)_{j i}\right.
$$

(just differentiate the products inside the square brackets and concentrate on one value of $i$ only). Applying the chain rule once again we find that (4.2.8) is equivalent to

$$
\sum_{j}\left(\sum_{k} \frac{\partial}{\partial x_{k}}\left(\operatorname{det} D_{x} h\right) \frac{\partial g_{k}}{\partial y_{j}}\right) \frac{\partial h_{j}}{\partial x_{i}}=\operatorname{det} D_{x} h \sum_{j, k} \frac{\partial^{2} h_{j}}{\partial x_{i} \partial x_{k}} \frac{\partial g_{k}}{\partial y_{j}} .
$$

Since, from (4.2.6), $\sum_{j} \frac{\partial g_{k}}{\partial y_{j}} \frac{\partial h_{j}}{\partial x_{i}}$ equals 1 or 0 depending on whether $i=k$ or not, the left hand side of (4.2.9) reduces to $\frac{\partial}{\partial x_{i}}\left(\operatorname{det} D_{x} h\right)$, which equals (see appendix $\left.\mathrm{A}\right)$

$$
\sum_{j, k} \frac{\partial^{2} h_{j}}{\partial x_{k} \partial x_{i}}\left[\operatorname{cofactor} \frac{\partial h_{j}}{\partial x_{k}}\right]
$$

But since $D_{y} g$ and $D_{x} h$ are each others inverses

$$
\left(\operatorname{det} D_{x} h\right)^{-1}\left[\text { cofactor } \frac{\partial h_{j}}{\partial x_{k}}\right]=\left[D_{y} g\right]_{k j}=\frac{\partial g_{k}}{\partial y_{j}} .
$$

In the time dependent case we get on multiplying (4.2.3.b) with $\operatorname{det} D_{x} h$

$$
\operatorname{det} D_{x} h\left(\frac{\partial m}{\partial t}+\nabla_{y} \cdot\left(D_{x} h v m\right)+\nabla_{y} \cdot\left(D_{t} h m\right)\right)=-\alpha n+b
$$

By the same calculations as before we find that

$$
\nabla_{y} \cdot\left(D_{x} h v m\right)=\nabla_{x} v n \text {. }
$$

So it remains to show that

$$
\operatorname{det} D_{x} h\left(\frac{\partial m}{\partial t}+\nabla_{y} \cdot D_{t} h m\right)=\frac{\partial n}{\partial t} .
$$

Differentiating $n(t, x)=m(t, h(t, x)) \operatorname{det} D_{x} h(t, x)$ for $t$ gives

$$
\frac{\partial n}{\partial t}=\left(\frac{\partial m}{\partial t}+\nabla_{y} m \cdot D_{t} h\right) \operatorname{det} D_{x} h+m \frac{\partial \operatorname{det} D_{x} h}{\partial t}
$$

Next we observe that (see appendix A)

$$
\frac{\partial}{\partial t} \operatorname{det} D_{x} h=\sum_{j, k} \frac{\partial^{2} h_{j}}{\partial x_{k} \partial t}\left[\text { cofactor } \frac{\partial h_{j}}{\partial x_{k}}\right]=\operatorname{det} D_{x} h \sum_{j, k} \frac{\partial^{2} h_{j}}{\partial x_{k} \partial x_{t}} \frac{\partial g_{k}}{d y_{j}}=
$$




$$
\operatorname{det} D_{x} h \sum_{j} \frac{\partial}{\partial y_{j}}\left(\frac{\partial h_{j}}{\partial t}(t, g(t, y))=\operatorname{det} D_{x} h \nabla_{y} \cdot D_{t} h(t, g(t, y))\right.
$$

Hence

$$
\frac{\partial n}{\partial t}=\operatorname{det} D_{x} h\left(\frac{\partial m}{\partial t}+D_{t} h \cdot \nabla_{y} m+m \nabla_{y} \cdot D_{t} h\right)
$$

which is equivalent to (4.2.10).

We have considered here the most general case, where the transformation relating $x$ and $y$ may be time dependent. One reason to use a time dependent $i$-state parametrization may be that this way we can sometimes bring equations which are time dependent into time independent form. However, time independent $i$-state transformations are more commonly called to help.

When $v$ is time independent an often useful (re)parametrization is given by the parameter $\theta$ indicating position along a characteristic as in subsection 4.1 , in conjunction with some $(k-1)$-dimensional variable $\xi$ indicating where this characteristic enters $\Omega$ through $\partial_{+} \Omega$ (see fig. 4.2.1). If there are no $i$-state jumps and all newborns necessarily start at $\partial_{+} \Omega, \theta$ is just what we call age. The map $g:(\theta, \xi) \mapsto x$ from (4.3.1) for this parametrization obviously has to be defined by

$$
\frac{d g}{d \theta}=v(g), \text { with } g(0, \xi)=x_{0}(\xi) .
$$

The great advantage of this choice of parametrization is that $\Omega_{\theta, \xi}$ is filled with nothing but parallel ordinary conveyor belts roling at unit speed, and distinguished by the parameter $\xi$. This is not only an easy image to deal with, it also makes for easy algebra and easy numerics. (In the next subsection we shall consider the generalization of this idea to the case of time dependent $v$. There we shall also check algebraically that the transformation defined by $(4.2 .11)$ indeed has the desired properties.)
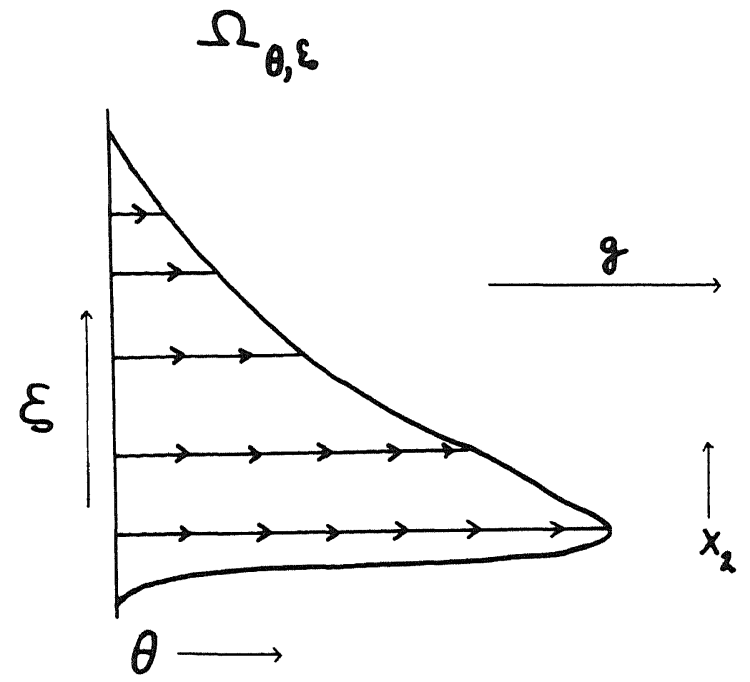

$\Omega_{x}$

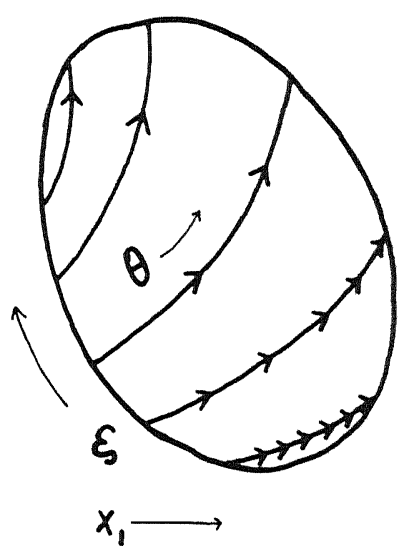

Figure 4.2.1 Reparametrizing the $i$-state $x$ with the time $\theta$ needed to move to $x$ along a characteristic starting at $x_{0}(\xi) \in \partial_{+} \Omega_{x}$, and a parameter $\xi$ indicating position along $\partial_{+} \Omega_{x}$. (The distance between the arrow points on the characteristics is supposed to correspond to one time unit.)

EXAMPLE 4.2.2: Size dependent reproduction in ectothermic animals. In 1.3 we have already seen how for this example under constant feeding conditions integration along characteristics could be used to arrive at a renewal equation for the population birth rate. The parameter $\theta$ characterizing position along the characteristic in this case was called $a$ for age. For animals born after $t=0$ the age-length relation is given by the function $L$ defined in (I.3.4.10). The inverse of this function was called A. (see I.3.4.11). If we define $a=A(l)$ also for characteristics which do not start at 
$\partial_{+} \Omega \times \mathbb{R}^{+}$(but at $\Omega \times\{0\}$ ), we can write an equation for the age distribution as

$$
\begin{aligned}
& \frac{\partial m}{\partial t}=-\frac{\partial m}{\partial a}-\mu m \\
& m(0)=\int_{0}^{\infty} \lambda(L(a)) m(a) d a
\end{aligned}
$$

EXERCISE 4.2.3: Derive (4.2.12) from (1.3.2.1) and vice versa.

EXAMPLE 4.2.4: The invertebrate functional response. If we apply the procedure indicated above to the predators busy handling prey from example 1.1 we get

$$
\begin{aligned}
& \frac{\partial m_{1}}{\partial t}=-\frac{\partial m_{1}}{\partial \theta}-\lambda(\tau(\theta)) m_{1} \\
& m_{1}\left(s, \tau_{p}(s)+\right)=q m_{1}\left(s, \tau_{p}(s)-\right) \\
& m_{1}(s, 0)=x g_{0}(s) n_{0}(s)
\end{aligned}
$$

At equilibrium

$$
\hat{m}_{1}(s, \theta)= \begin{cases}x g_{0}(s) \hat{n}_{0}(s) e^{-\mu \theta} & \text { for } \theta<\tau_{p}(s) \\ x g_{0}(s) \hat{n}_{0}(s) q e^{-\mu \tau_{p}(s)} & \text { for } \theta>\tau_{p}(s)\end{cases}
$$

Therefore

$$
\int_{\Omega_{s, \tau}} \hat{n}_{1} d s d \tau=\int_{\Omega_{1,0}} \hat{m}_{1}(s, \theta) d s d \theta=\int_{0}^{s_{m}} \hat{n}_{0}(s) x g_{0}(s)\left[\mu^{-1}\left(1-e^{-\mu r_{\rho}(s)}\right)+\tau_{e} q e^{-\mu \tau_{\rho}(s)}\right] d s,
$$

making (4.1.9b) reduce to

$$
\int_{0}^{s_{m}} \hat{n}_{0}(s)\left[1+x g_{0}(s)\left(\mu^{-1}\left(1-e^{-\mu \tau_{\rho}(s)}+\tau_{e} q e^{-\mu \tau_{p}(s)}\right)\right] d s=1 .\right.
$$

The factor following $x g_{0}(s)$ can be interpreted as the mean time a predator spends on sighted prey. $\mu^{-1}\left(1-e^{-\mu \tau_{\rho}(s)}\right)$ represents the mean time spent on pursuits, both the successful and the unsuccessful ones. $q e^{-\mu \tau_{p}(s)}$ is the probability that a prey that is sighted is also caught.

The functional response can be calculated from

$$
\int q \hat{m}_{1}\left(s, \tau_{p}(s)\right) d s=\int_{0}^{c} x g_{0}(s) q e^{-\mu \tau_{p}(s)} \hat{n}_{0}(s) d s .
$$

In the following exercises we shall not only effect a transformation of the $i$-state of the PPP-problem in the manner indicated above, but we shall also replace the partial differential equation in three variables, $t, x$ and $y$, for the predator patches by an equivalent pair of partial differential equations in two variables only. This transformed system forms the starting point of the calculations by Sabelis in part B.

EXERCISE 4.2.5: Transform the $p$-equation, including boundary conditions, for the predator patches by replacing the variables $x$ and $y$ by the variables $\theta_{2}$, "age" of the predator patch, and $\xi$, the value of $x$ at which the first predator arrived. Give also a formula for the rates $R$ and $S$ at which free searching, patchless prey and predators are produced.

EXERCISE 4.2.6: Transform the $p$-equations for both the predator and prey patches by replacing for the prey patches the variable $x$ by $\theta_{1}$, the "age" of the prey patch, and for the predator patches $\xi$ by $\theta_{1}$, age of prey patch at which the first predator arrived. Give also formulae for $R$ and $S$.

EXERCISE 4.2.7: Replace $\theta_{2}$ from the previous exercises by the time $\tau$ till the predator patch ceases its existence, due either to resource exhaustion or extinction of the prey. Again give formulae for $R$ and $S$.

EXERCISE 4.2.8: Let $R$ and $S$ in the previous exercise be calculated as

$$
R=\int \rho\left(\theta_{1}\right) m_{2}\left(\theta_{1}, 0\right) d \theta_{1}+m_{1}\left(a_{m}\right) x_{m}, S=\int \sigma\left(\theta_{1}\right) m_{2}\left(\theta_{1}, 0\right) d \theta_{1},
$$


where $m_{1}$ denotes the density function over $\Omega_{\theta_{1}}$ and $m_{2}$ the density function over $\Omega_{\theta_{1}, \tau}$. Now define

$$
r(\tau)=\int \rho\left(\theta_{1}\right) m_{2}\left(\theta_{1}, \tau\right) d \theta_{1}, \quad s(\tau)=\int \sigma\left(\theta_{1}\right) m_{2}\left(\theta_{1}, \tau\right) d \theta_{1} .
$$

Derive partial differential equations and boundary conditions for $r$ and $s$.

Observe that the equations for $n_{0}$, the number of empty patches, $m_{1}, r$ and $s$ together form a complete state space model for the within patch "black box" as far as the calculation of $R$ and $S$ is concerned. If we couple these equations with $p$-equations for the free searching prey and predators the end result is a complete, autonomous model. The course of the state of this reduced model over time is sufficient information to calculate the time course of the state of the original PPP-model from an integration along characteristics.

\subsection{Following cohorts through time}

When $v$ does depend on $t$, implicitly or explicitly, the characteristics in $\Omega$ are no longer constant curves but change over time. Yet, if newborns only start at $\partial_{+} \Omega$, it may still be useful to characterize an individual by its age $a$ and its state at birth, again parametrized by $\xi$. The reparametrization connecting the $i$-state $x$ of an individual to the characterization of that same individual by a pair $(a, \xi)$ is necessarily time dependent i.e. the map $g:(a, \xi) \mapsto x$ depends on $t$. As $g$ plays an essential role in the determination by $a$ and $\xi$ of any $x$-dependent quantities like individual birth rate, the mechanism by which the frequency distribution $m$ over the space $\Omega_{a, \xi}$ of reachable $(a, \xi)$-pairs changes is time dependent as well. If we want a description of our $p$-system in which any time dependence is as usual accounted for by a dependence on the $p$-input we have to include $g$ in our population description as a necessary adjunct to the frequency distribution $m$. Together the pair $(m, g)$ indeed qualifies as the $p$-state of yet another alternative representation of our population. The differential generator of the corresponding $p$-system then consists of a differential equation which updates $m$ plus a differential equation doing the same job for $g$. We propose to call this idea after its originatrix the Murphy-trick (Murphy, 1983a)

The differential equation for $m$, given $g$, can easily be written down from first principles. The differential equation for $g$ can immediately be written down by observing that $a$ may be considered a position along a conveyor belt moving with speed one. Following a point on the surface of the conveyor belt corresponds to following one individual through its life. If we follow a representative individual born at $t_{0}$ in state $x_{0}(\xi)$ and having state $x$ at time $t=t_{0}+a$

$$
g\left(t_{0}+a, a, \xi\right)=x\left(a, x_{0}, t_{0}\right) \text { and } \frac{d x}{d a}=\frac{d g}{d a}=\frac{\partial g}{\partial t}+\frac{\partial g}{\partial a} .
$$

Since also

$$
\frac{d x}{d a}=v\left(t_{0}+a, x\right)
$$

we have

$$
\frac{\partial g}{\partial t}+\frac{\partial g}{\partial a}=v(t, g), \quad g(0, \xi)=x_{0}(\xi)
$$

(NB (i) when $\operatorname{dim} \Omega>1, g$ is vector-valued, (ii) the combined equation for $g$ and $m$ will in general be nonlinear)

REMARK 4.3.1: In our above exposition of the Murphy trick we restricted ourselves to individuals born after $t=0$. The individuals present at $t=0$ can for example be described by their state at $t=0$ plus (an analogue of) the function $g$ telling their state at $t$ in dependence on both $t$ and their state at $t=0$. The two sets of coordinates, $\xi$ and $a$, and state at $t=0$ and $t$, have in common that they are especially adapted to integration along characteristics.

EXAMPLE 4.3.2: Size dependent reproduction in ectothermic animals. If food density $x$ is not constant the analogue of (4.2.11) - (4.2.12) becomes (NB $g$ in (4.3.2a) corresponds to $v$ from (4.3.1))

$$
\begin{aligned}
& \frac{\partial l}{\partial t}+\frac{\partial l}{\partial a}=g(x, l), \quad l(0)=l_{b}, \\
& \frac{\partial m}{\partial t}+\frac{\partial m}{\partial a}=-\mu m, \quad \text { for } l(a) \leqslant \bar{l}(x), \\
& m(a)=0, \quad \text { for } l(a)>\bar{l}(x), \\
& m(0)=\int_{0}^{\infty} \lambda(x, l(a)) m(a) d a
\end{aligned}
$$


One reason to call attention to the Murphy trick is that it seems to hold considerable potential for the development of numerical procedures for coping in a uniform way with a large class of structured population models. A second reason is that Murphyzed equations often provide a good starting point for further transformation procedures like "linear chain trickery" (see e.g. Nisbet, Gurney, Blythe \& Metz (1985); IV 5.2 and the contribution of Gurney, Nisbet \& Blythe in these notes introduce the concept for age resp. physiological age dependent models.) The final, and most important reason is that it enables us to cope in an easy manner with situations in which the $p$-distribution is concentrated on some lower dimensional manifold in $\Omega$, as discussed in section 5 .

* INTERLUdE 4.3.3: The algebra of transforming to age and $i$-state at birth

We follow the notation of subsection 4.2 , with some minor adaptations. So $h(t, x)$ denotes the inverse of $g(t, a, \xi)$ with respect to the last two variables, i.e.

$$
(\underset{\xi}{a})=h(t, g(t, a, \xi)) \text {. }
$$

From (4.2.3a)

$$
m(t, a, \xi)=n(t, g(t, a, \xi)) \operatorname{det} D_{(a, \xi)} g(t, a, \xi)
$$

and from $(4.2 .3 \mathrm{c})$ the speed in the $(a, \xi)$ plane equals

$$
w(t, a, \xi)=D_{x} h\left(v-\frac{\partial g}{\partial t}\right) .
$$

But

$$
D_{x} h=\left(D_{a} g, D_{\xi} g\right)^{-1}=\left(v-\frac{\partial g}{\partial t}, D_{\xi} g\right)^{-1},
$$

where the last equality follows from (4.3.1). Therefore

$$
\sum_{j}\left(D_{x} h\right)_{i j}\left(v_{j}-\frac{\partial g_{j}}{\partial t}\right)= \begin{cases}1 & \text { for } i=1 \\ 0 & \text { for } i \neq 1\end{cases}
$$

EXERCISE 4.3.4: Derive (1.3.2.1) from (4.3.2) and vice versa.

EXERCISE 4.3.5: Murphyze the cell kinetics model from example 1.4. Also derive the ordinary $p$-equations from the Murphy-type equations. Assume that the division rate $b$ is not time dependent.

* EXERCISE 4.3.6: Formulate possible converses of interlude 4.3.3.

EXERCISE 4.3.7: How should the concept of Murphy equations be extended to cover the cell kinetics model from I.4? Write down the Murphy equations both for the probability per unit of time and the stochastically predetermined size assumptions about the division process.

Hint: There are two possible approaches. One is to keep the characterization of individuals by age and $i$-state at birth. In that case one should follow the lead of exercise 4.3.5 and as the last step integrate over the spurious variable age. The other possibility is to use a quantity analogous to $\theta$ from the previous section, as ones $i$-description. $\theta=h(x)$ can for example be defined as the age a hypothetical individual that started at $\partial_{+} \Omega$ would have if it now has size $x$.

\section{About delta-functions and related topics}

In section 3 we already mentioned a few times that some of the formulae there were not completely general, unless we allowed the expressions involved to contain delta- "functions" to represent sharp localized effects in a formalism that otherwise only allows smoothly distributed ones. In such a formalism delta-functions are the natural representations of point masses versus mass densities, instantaneous death probabilities versus death rates, etc. In this section we shall subject this suite of ideas to a closer scrutiny.

The main advantage of the delta-function formalism is its conciseness and unifying generality. This also makes the delta-function formalism an ideal tool for expressing our biological assumptions. The penalty which we have to pay is that in each specific instance we have to find out what the formalism exactly means for our equations, i.e. we have to translate our formal expressions containing delta-functions into less slim looking but concretely manageable ones. In 
this section we shall give some of the rules for achieving this. We shall mainly concentrate on the one-dimensional case. Only in the last subsection we shall demonstrate by means of examples the extension to higher dimensional $i$ state spaces.

\subsection{The delta function formalism}

In these notes we shall take the line that delta-functions are terms that we put into formal integral expressions in order to have one formalism for quantities which in conceptually related cases can be calculated as classical integrals, but not in the case under consideration. For such a line to make sense the two kind of cases should at least be related through some kind of limit argument.

EXAMPLE 5.1.1: If we define for $c \in C[-1,1]$,

$$
f(c)=\int c(x) w(x) d x,
$$

where $w$ is some integrable weight function, then $f$ defines a continuous linear map from $C([-1,1])$ to $\mathbb{R}$. Such a map is called a continuous linear functional on $C([-1,1])$. Unfortunately not every such functional can be represented by (5.1.1) for some $w$. The linear functional $g_{0}(c)=c(0)$ provides an immediate counterexample. However, $g_{0}(c)$ and similar linear functionals can be obtained as limits of expressions of the form (5.1.1). For example, let

$$
w_{i}(x)=\left\{\begin{array}{ll}
\frac{1}{2} i & \text { for } x \in\left(-i^{-1}, i^{-1}\right) \\
0 & \text { elsewhere }
\end{array},\right.
$$

then

$$
g_{0}(c)=\lim _{i \rightarrow \infty} \int c(x) w_{i}(x) d x
$$

The formal limit ${ }^{*}$ of the sequence $w_{i}$, and of any other similar sequence, like

$$
w_{i}(x)=\left\{\begin{array}{ll}
2 i^{2}\left(i^{-1}-x\right) & \text { for } x \in\left(0, i^{-1}\right) \\
0 & \text { elsewhere }
\end{array},\right.
$$

such that (5.1.2b) holds for any $c \in C[-1,1]$, is denoted as $\delta$, so that we may write

$$
g_{0}(c)=\int c(x) \delta(x) d x .
$$

As an indication of the power of this formalism we write for $a \in(-1,1)$

$$
\begin{aligned}
& \delta_{a}(x):=\delta(x-a), \\
& g_{a}(c):=\int c(x) \delta_{a}(x) d x,
\end{aligned}
$$

which implies

$$
g_{a}(c)=c(a) .
$$

For $a= \pm 1(5.1 .3)$ is no longer well defined (to see this consider the two sequences (5.1.2a) and (5.1.2a): for the first sequence $(5.1 .2 \mathrm{~b})$ would give us $g_{a}(c)=\frac{1}{2} c(a)$, and for the second sequence $\left.g_{a}(c)=c(a)\right)$. In order to include these cases as well we shall modify our definition of $\delta$ to include the requirement that in any formula in which $\delta$ occurs we shall always confine the attention to weighting functions which have their support contained in $[-1,1]$. With this modification (5.1.3) and (5.1.4) imply (5.1.5) also for $a= \pm 1$.

REMARK 5.1.2: Delta functions are very much a scientist's tool. The real mathematical objects are not the deltafunctions $\delta_{a}$ but the delta functionals $g_{a}$. The latter objects are the subject of the theory of distributions as developed by Schwartz (1950, 1951) (see also Mikusinsky (1959), Gelfand \& Shilov (1964), Rudin (1973), Trèves (1967) and Yosida (1980)) which has the specific purpose to cope in a mathematically consistent way with delta-functions and the like. However, we do not intend to make the connection with this theory here in any but the most handwaving manner.

- For mathematicians only: Formal limits can be defined as equivalence classes of sequences, under the equivalence relation "leading to the same limit result in the formulae under consideration". 
The simplest use of delta-functions in the specification of the $p$-differential generator is precisely in the manner of the following example: the distribution of the "touch down" positions after an $i$-state jump is assumed to contain one or more (multiples of) delta-functions.

EXAMPLE 5.1.3: Multiplication by division. The cell proliferation model from 1.4, where division was assumed to be into two equal parts is but a special case of the more general cell division model expounded in section 3.3, formula (3.3.3), in which $f(\theta)=\delta\left(\theta-\frac{1}{2}\right)$, leading to a birth rate at $x$ of

$$
\int_{0}^{1} 2 f(\theta) \theta^{-1} \alpha_{d}\left(\frac{x}{\theta}\right) n\left(\frac{x}{\theta}\right) d \theta=4 \alpha_{d}(2 x) n(2 x),
$$

in accordance with (I.4.2.1).

Some slightly more subtle uses of delta-functions will be explicated in the next subsection.

\subsection{Delta-functions and transition conditions}

Life becomes more complicated when we write delta functions not explicitly under an integral sign but only implicitly, like in the right hand side of a differential equation.

We shall start considering the case where a disappearance term $\alpha$ contains a delta function. Suppose we write

$$
\alpha=\alpha_{0}+\beta \delta(x-a)
$$

with $\alpha_{0}$ bounded, i.e. we assume that apart from a bounded death rate we also have a concentrated occurrence of deaths at $x=a$. The question then is how many deaths occur at $x=a$ and what does (5.2.1) mean for the $p$ differential generator? To answer this question we consider again the sequence $w_{i}$ from (5.1.2a) that converges to a delta-function. To pass the interval $\left(a-\frac{1}{2} \epsilon, a+\frac{1}{2} \epsilon\right)$ the individual needs a time $\epsilon /|\nu(a)|+o(\epsilon)^{*}$. During that time the disappearance probability per unit of time is $\beta \epsilon^{-1}+O(1)$. So the probability of still being present aftèr having passed the critical interval is $\exp (-\beta /|v(a)|+o(1)$ ), or in the limit for $\epsilon \downarrow 0$, for $v(a)>0$ (remember that $\phi$ denoted the flux)

$$
\phi(a+)=\exp (-\beta / v(a)) \phi(a-)
$$

or, since $\phi=v n$,

$$
n(a+)=\exp (-\beta / v(a)) n(a-) .
$$

Some thought shows that (5.2.3) holds for $v(a)<0$ as well.

EXERCISE 5.2.1: Show that the second result of the previous calculations is independent of the specific choice of the sequence of weighting functions by which we represent $\delta$.

Hint: Consider only positive weighting functions. Calculate an explicit expression for the probability of surviving through an interval of length $\epsilon$ around $a, \epsilon$ fixed. Take the delta-function limit, and only then let $\epsilon \downarrow 0$. (The answer to this exercise can be found in example 6.2.3.

In the limit $n$ is no longer differentiable or even continuous at $a$. So $n$ no longer satisfies a partial differential equation in the whole interior of $\Omega$ but only in the interior of the two subsets $x<a$ and $x>a$. Conditions like (5.2.2) and (5.2.3) that relate two subsets of $\Omega$ over a boundary at which $\phi$ or $n$ makes a jump are called transition conditions. We have already met an example of a transition condition in the invertebrate predator model where a strike led to an instantaneous probability of escape as opposed to the escape probability per unit of time during pursuit (formula 4.1.5c).

Another possibility is that the (re)appearance term $b$ contains a delta function at $a$ like

$$
b(x)=b_{0}+c \delta(x-a) .
$$

Applying the same reasoning as before we find the transition condition

$$
\phi(a+)=\phi(a-)+c
$$

* The notation $y=o(x)(y=O(x))$ means that $y / x \rightarrow 0(y / x$ stays bounded $)$. 
or equivalently

$$
n(a+)=n(a-)+c / v(a) .
$$

In the special case that $a$ lies exactly at the boundary of $\Omega$, say the left hand boundary and $v>0$, and where there is no influx of mass across the boundary, (5.2.5) reduces to

$$
v(a) n(a+)=v(a) n(a-)+c=c,
$$

where we rewrote the usual boundary condition in the absence of influx as

$$
n(a-)=0 \text {. }
$$

So the delta-function formalism unifies the two hitherto separate ways in which mass may (re)appear.

It may come as a surprise that taking limits in the defining formula of a p-differential generator may lead to side conditions. However what really matters is the convergence of the associated semigroup (which we did not prove!) and not the subtleties of the connections between semigroups and generators. A more detailed account of this problem may be found in appendix 6A. A second manner in which what effectively amounts to a side condition was generated through a limiting argument pertaining to the interior of $\Omega$, can be found in exercise 1.3: A death rate $\epsilon /\left(a_{\max }-a\right)$ naturally restricts age $a$ to $\left[0, a_{\max }\right)$. If we let $\epsilon$ go to zero the restriction of $a$ remains, but in the interior of $\Omega$ there are no visible remnants of the mechanism by which this restriction came about.

EXERCISE 5.2.2: In the age as well as size dependent cell proliferation model from example 1.4 assume that a stochastic size threshold for division model applies like expounded in I.4.1, except that the division is only allowed to occur in cells older than age $a_{0}$. What does the $p$-equation look like? (Assume that the distribution of the size threshold and the growth pattern are such that some cells already reach the threshold before age $a_{0}$.)

* EXERcise 5.2.3: One would like to have a good distributional interpretation of partial differential equations with distributional source and sink terms. However, this is actually rather complicated in the sink case. In the source case the following calculations are illustrative.

Let $n$ satisfy

$$
\frac{\partial n}{\partial t}+\frac{\partial}{\partial x}(v n)=f(t, x)
$$

with $\nu$ independent of time. Derive a differential equation for the flux $\phi=v n$. Solve this differential equation by the method of characteristics on the assumption that $\phi\left(x_{0}\right)=b(t)$. Now let $f(t, x) \rightarrow c(t) \delta(x-a)$. What does the formula for $\phi$ become? In this case there is no problem with a distributional interpretation as $\phi$ is just the sum of a Heaviside function and a smooth function, and the $\delta$-"function" is precisely the distributional derivative of the Heaviside component. (This also was the reason why we stated our results first in terms of $\phi$ and only afterwards in terms of $n$.)

\subsection{Delta-functions in initial conditions}

Often we wish to allow delta-functions as initial conditions. (For example, we may wish to start a predation experiment with a population of totally satiated predators.) The natural way to do this would be to work with a $p$-state space rich enough to contain such objects. (Of course such objects can never belong to the domain of the differential generator as derived in section 3. Chapter II discusses how such a differential generator can still uniquely define a semigroup on the total $p$-state space.)

* REMARK 5.3.1: The best move in many cases probably is to work in the space of Borel measures on $\Omega$, provided with the weak * topology derived from its interpretation as the dual of $C(\Omega)$. This space is just sufficiently rich to contain all objects corresponding to even our wildest thought experiments, and nearness in the weak * topology is an immediate counterpart of being close as far as observations go. An example of this approach may be found in Heijmans' treatment of our invertebrate predator example (Heijmans, 1984d).

However, often it is easier for technical reasons to restrict the $p$-state space to some space of better behaved functions, like the Lebesgue integrable or continuous ones. One way to smuggle in delta-functions in such a framework exploits the observation that usually the state jumps have a sufficient smoothing action for the delta-function to have disappeared among the "first generation" descendants of the delta-function wise concentrated "zero'th generation". In that case we can simply treat the zero'th generation as a separate entity. 
ExAMPLE 5.3.2: Assume that in the predator of 1.2.5, i.e. the predator with negligible handling time, we start our experiment with $N_{0}$ predators at satiation $s_{m}$, the maximum of the gut capacity. Than we can formulate the $p$-problem as

$$
\begin{aligned}
& \left\{\begin{array}{l}
\frac{d S}{d t}=-f(S), \quad S(0)=s_{m}{ }^{\prime} \\
\frac{d N}{d t}=-x g(S), \quad N(0)=N_{0}
\end{array}\right. \\
& \left\{\begin{array}{l}
\frac{\partial n}{\partial t}=-\frac{\partial f n}{\partial s}-x g(s) n+x g(s-w) n(s-w)+x g(S) N(S) \delta(s-S-w) \\
-f\left(s_{m}\right) n\left(s_{m}\right)=\int_{s_{m}-w}^{c} x g(s) n(s) d s+\chi\left(S>s_{m}-w\right) x g(S) N \\
n(s, 0)=0 .
\end{array}\right.
\end{aligned}
$$

where $\chi(A)$ denotes the indicator function of the event between the brackets (i.e. $\chi\left(S>s_{m}-w\right)=1$ if $S>s_{m}-w$ and zero otherwise).

In example 5.3.2 we obtain a piecewise continuous first generation. If we wish continuity we should also keep the first generation separate, etc. (provided $g$ is sufficiently smooth).

Formula (5.3.1a) describes the fate of a delta function of initial size $N_{0}$ when we follow it along a characteristic.

EXERCISE 5.3.3: Replace (5.3.1b) by a separate (formal) partial differential equation for the first generation distribution and a further partial differential equation for the second plus higher generations distribution. Next replace the equation for the first generation by two partial differential equations on contiguous, moving, domains, coupled by a transition condition. Solve these equations by integration along their characteristics. This solution may be used to account for the first generation in case we only wish to confine our dealings with $p$-states to continuous $i$-state distributions.

EXERCISE 5.3.4: Equation (5.3.1a) does not contain a dilation term. Such in contrast to the equations which we get when following the local behaviour of an ordinary $p$-distribution along a characteristic. Explain this phenomenon, in connection to our definition of a delta function as a formal limit of a sequence of progressively more concentrated $p$ distributions.

Hint: Ask yourself what happens to the sequence of weight functions from (5.1.2a) when they move along with the conveyor belt.

EXERCISE 5.3.5: How should the equations of the example from I.3 (size dependent reproduction in ectothermic animals) be modified if we start our population with $N_{0}$ neonates?

\subsection{Delta-functions in more dimensions}

In the example we have already encountered a number of applications of the delta-function formalism in higher dimensional state spaces, like the transition condition at the passage from pursuit to eating in our predator model. All these examples had in common that the delta-functions were essentially one dimensional, i.e. they could be written as a product of a delta-function in one direction times an ordinary function in the other directions (and the deterministic flow $v$ was transversal to those "other" directions). Higher dimensional delta-functions which are integrated over a variable of the same dimension can be treated exactly as in the one-dimensional case. As a rule essentially higher dimensional delta-functions do not appear in the sink term $\alpha n$. Our concern therefore should be higher dimensional delta-functions in the source term $b$ : these do not disappear but move on with the stream in the same manner as delta-functions in initial conditions. We shall not try to give a general survey of all the possibilities but restrict ourselves to two examples. (In both examples the delta-functions appear in the boundary condition at $\partial_{+} \Omega$. The analysis from subsection 5.2 makes clear that such boundary conditions can be interpreted as delta-function valued source terms contiguous to the boundary.)

EXAMPLE 5.4.1: Size dependent reproduction in ectothermic animals, finale. Using the delta-function formalism the $p$ equations for example 1.2 from the start of this chapter are

$$
\frac{\partial}{\partial t} n(t, l, a)+\frac{\partial}{\partial a} n(t, l, a)+\frac{\partial}{\partial l}(g(x, l) n(t, l, a))=-\mu(l, a) n(t, l, a),
$$




$$
n(t, l, 0)=\delta\left(l-l_{b}\right) \int_{\Omega} \lambda(x, l) n(t, l, a) d l d a,
$$

where (5.4.1b) expresses the fact that newborns all have length $l_{b}$. The action of the two dimensional conveyor belt symbolized by (5.4.1a) moves the delta function appearing in the side condition (5.4.1b) bodily into the interior of $\Omega$. The result is a one dimensional delta-function which lives on a line in two-dimensional space. This is nice as an image goes but it does not help in analyzing our population model. It is here that the Murphy trick from subsection 4.3 brings relief: we project all $p$-mass on the age axis and introduce a separate equation to calculate the instantaneous age-length relation. The resulting $p$-equation we encountered already as (4.3.2).

EXAMPLE 5.4.2: Colony size distribution in the diatom Asterionella. Till now in our discussion of this example we have concentrated on the main sequence of colony sizes $2^{k}, k=0,1,2, \cdots$. However, other colony sizes do occur even if relatively rarely. Since synchronous division is fairly well documented this must be due to a breakage mechanism in which the bonds are less than completely dependent. This is also in accordance with intuition. If we wish to relax the assumption of complete dependence we are left with a staggering choice of possible mechanisms. As the observed colony size distributions are the only data available on which we can decide between the various alternatives it is of no use to explore even a moderate range of possibilities. We shall therefore only consider the extreme opposite of complete dependence as a possibly useful null-model and assume that the bonds break completely independently.

If the bonds are not completely dependent there is no longer a one to one correspondence between the age of the youngest bond and cell age. Therefore we introduce a different parametrization of the $i$-state space. To this end we observe that in a colony the bonds are ordered in a linear sequence. So we can choose as an $i$-state the cell age $a_{0}$ plus the ages $a_{1}$ to $a_{r}$ of the various bonds counted from left to right. Here we may think of "left" and "right" as being determined by which of the two observationally indistinguishable flat sides of a colony happens to be up when it is put onto the microscope slide; the arbitrariness of this procedure does not matter as the breakage and division mechanisms are necessarily invariant under the operation of flipping over a colony and all its descendants. The corresponding $p$-state is denoted as $n_{r+1}\left(a_{0}, a_{1}, \cdots, a_{r}\right), r=0,1, \cdots(r+1$ is the colony size), with the convention that $n_{r+1}\left(a_{0}, a_{1}, \cdots, a_{r}\right)=n_{r+1}\left(a_{0}, a_{r}, \cdots, a_{1}\right)$.

If we denote the age specific division rate as $d$ and the bond-age specific breaking rate as $b$ we get as our $p$-equations

$$
\begin{aligned}
& \frac{\partial n_{r+1}}{\partial t}+\sum_{i=0}^{r} \frac{\partial n_{r+1}}{\partial a_{i}}=-d\left(a_{0}\right) n_{r+1}-\sum_{i=1}^{r} b\left(a_{i}\right) n_{r+1} \\
& +\sum_{j=r+1}^{\infty} \int_{0}^{\infty} \cdot \int_{0}^{\infty} b\left(a_{r+1}\right)\left[n_{j+1}\left(a_{0}, a_{1}, \cdots, a_{r}, a_{r+1}, \cdots, a_{j}\right)\right. \\
& \left.+n_{j+1}\left(a_{0}, a_{r}, \cdots, a_{1}, a_{r+1}, \cdots a_{j}\right)\right] d a_{r+1} \cdots d a_{j}
\end{aligned}
$$

with the side conditions

$$
n_{r+1}\left(0, a_{1}, \cdots a_{r}\right)= \begin{cases}\delta\left(a_{1}\right) \delta\left(a_{3}\right) \cdots \delta\left(a_{r}\right) \int_{0}^{\infty} d\left(a_{0}\right) n_{(r+1) / 2}\left(a_{0}, a_{2}, \cdots, a_{r-1}\right) d a_{0} & \text { for } r+1 \text { even } \\ 0 & \text { for } r+1 \text { odd }\end{cases}
$$

Just as in the previous example the delta-functions are moved bodily into the interior of $\Omega$ along the lines $a_{1}=a_{2}=. .=a_{r}$. While moving they decrease in size due to bonds breaking, at the same time spawning lower dimensional delta-functions corresponding to the fragmentation products. (This picture may seem hopelessly complicated. Yet, in chapter IV we shall extract some information from this model albeit by totally different methods.)

EXERCISE 5.4.3: Try to rewrite this model without using delta-functions. Introduce to this end a special notation for the $p$-masses on the various diagonal planes supporting delta-functions, and write equations for the movement of all these separate components of the $p$-state.

(Hint: we didn't dare try doing this exercise ourselves!)

\section{Limiting processes and model simplification}

\subsection{Introduction: the role of limit arguments}

Structured population models are considerably more resistant to mathematical analysis than their nonstructured coun- 
terparts. A mathematical toolbox for dealing with these models is only on the verge of being developed. Chapters II IV, V and VI give some indication of the state of the art. Numerical work also tends to be extremely time consumini except in certain special cases (see e.g. the contributions by Goudriaan and by Gurney, Nisbet and Blythe in part B) Finally these models generally are relatively parameter rich which poses a problem to the experimental and theoretica biologist alike who respectively have to supply the parameter values for particular applications or should explore th parameter space to get a general idea about the possible dynamics. Therefore, when the initial modelling stage is ove it is of the utmost importance to simplify one's model as far as possible. How well a simplification performs shoulc be judged mainly on the extent to which the connection between population phenomena and individual mechanisms i preserved, i.e. a good simplified model should match the original model's prediction to an extent determined by th application in mind while at the same time keeping to the essentials of the mechanistic assumptions of the origina model.

The main simplification technique is through limiting procedures. That is, we observe that some parameters art small or large relatively and then consider a limit in which these parameters are set equal to zero or infinity. In thi: final section we shall give an indication of some of the possibilities.

Some examples of limiting procedures we have already encountered in section 5: our approach to delta-function: was essentially through appropriate limit arguments.

EXAMPLE 6.1.1: Multiplication by division. In this example we let the density $f$ of the relative size after division $t$ approach $\delta\left(\theta-\frac{1}{2}\right)$. The resulting limit model may be a good approximation if the variance of $f$ is small as it usually is. It both has fewer parameters and allows an easier calculation of the stationary size distribution.

There is one snag. In the practically important special case of exponential individual growth the limit model allows a unique stationary size distribution, but the population does not converge to it in the course of time, (see II.11): whereas for nonzero variance of $f$ such convergence does occur, (see VI.5 and Heijmans (1984b)). Still the stationary size distribution for the limit model can be shown to be a good approximation to the stationary size distribution for small variances. (But the lack of a smoothing action in the limit model may well play havoc with some numerical procedures for dealing with nonlinear variants of the model.)

The example shows that there may be various subtleties involved where it comes to the convergence of the associated semigroups. In the case of exponential individual growth the semigroup certainly cannot converge uniformly for all positive time. Appendix $6 \mathrm{~A}$ gives a short introduction to the mathematical aspects of the convergence problems. In the main text we shall confine ourselves to formal calculations, while stressing the biological side of the argument. Nowhere shall we deal generally and in depth with the kind of pathologies sketched above. But we trust that our intuition will tell us how to deal with them in any specific instance.

\subsection{Time scale arguments}

Time scale arguments provide one of the most, if not the most, important ways of simplifying models.

EXAMPLE 6.2.1: Size dependent reproduction in ectotherms. In the model of this name from chapter I we assumed eventually that individual food supply $x$ was coupled dynamically to the $p$-state. For model parameters based on laboratory experiments with Daphnia magna it turned out that food dynamics was extremely fast compared to the population reaction. Therefore in the numerical simulations we assumed that the (constant) food supply to the population $\alpha$ was always in equilibrium with the feeding rate of the Daphnia population and the (linear) rate of food deterioration $\beta x$.

This use of a time scale argument is completely analogous to that in ordinary differential equation population models Some population state variables are assumed to have relaxation times grossly different from those of the remaining state variables so that we can treat the slow variables as constants on the time scale of the fast variables, and the fasi variables as being permanently in equilibrium on the time scale of the slow variables. In the example the fast subspace was one dimensional. An example with an infinite dimensional fast subspace is provided by our model for the invertebrate functional response from I.2, where we assumed the time scale on which the satiation distribution equilibrates to be fast relative to the prey dynamics (and to individual growth; compare the use of the functional response in I.3 size dependent reproduction in ectothermic animals!).

In structured population models it is also possible to use time scale arguments on the individual level, as a mean: of lowering the dimensionality of the $i$-state space $\Omega$. An example is provided by the initial neglecting of prey handlin times in our invertebrate predator model. 
EXERCISE 6.2.2: Derive formally equation (I.2.5.5) from equation (4.1.8).

Below we shall consider three examples in which individual and population time scales are combined in a nontrivial manner. The important message to be derived from these examples is that in such more complicated cases one should carry out the simplification in detail rather than try to specify the simple model direct on an $a d$ hoc basis.

\section{EXAMPLE 6.2.3: Egg eating predators.}

Consider an age structured prey population obeying the balance law

$$
\frac{\partial n}{\partial t}+\frac{\partial n}{\partial a}=-\alpha n, \quad n(0)=b
$$

where $\alpha$ denotes the relative death rate and $b$ the birth rate. For the sake of the exposition we assume temporarily that the only cause of death is predation. So, assuming as a start a simple law of mass action interaction we may put

$$
\alpha(t, a)=\beta(a) p(t),
$$

where $p$ denotes the density of predators. $\beta$ is an age specific vulnerability index (the variation in $\beta$ with age may be due for example to the differences in escape probabilities of differently aged prey).

Some species do only predate on individuals of a prey population which are in an early stage of their life cycle (e.g. eggs or larvae or molluscs with a shell sufficiently thin to drill through). In that case $\beta$ will differ from zero only for $0 \leqslant a<\epsilon$, where $\epsilon$ is small. As an idealization one may want to put $\epsilon=0$, i.e., only individuals of age zero are vulnerable to predation so that, effectively, predation only affects the rate of recruitment to the prey population. This idea was first introduced by Gurtin \& Levine (1979), who just made an ad hoc assumption about the predation effect on recruitment, not based on any more detailed model for the predation process. This assumption was later criticized by Thompson, DiBiasio \& Mendes (1982). However, the alternative which these authors proposed was equally lacking a mechanistic basis (they argued as if they were concerned with numbers whereas they were dealing with rates). The right formulation from a mechanistic viewpoint is contained already in the general arguments from section 5 . In order for the predation effect not to become negligible when $\epsilon$ goes to zero we have to assume that the integral of $\beta$ stays equal to some constant, say $\theta$. Then $\beta$ itself converges to $\theta$ times a delta-function at zero, and by (5.2.3) we arrive at

$$
n(0+)=b \exp (-\theta p) \text {, }
$$

which is the correct recruitment rate as modified by "egg predation". The factor $e^{-\theta p}$ is the probability that an egg survives the predation window, the risk of succumbing to predation being $1-e^{-\theta p}$. The quantity $\theta$ measures the intensity of the predator prey interaction. It can be interpreted as the effective "deadly" area/volume surrounding one predator (remember $p$ was the number of predators per unit of area/volume).

Before going on to the next step, the incorporation of a functional response, we shall derive (6.2.3) again from first principles, as we need the intermediate steps at a later stage. We start by setting

$$
\beta(a)=\epsilon^{-1} \zeta(a / \epsilon),
$$

where $\zeta$ is some nonnegative function with support ${ }^{*}$ in $[0,1]$ and

$$
\int_{0}^{1} \zeta(\tau) d \tau=\theta
$$

to express the fact that $\beta$ may only differ from zero on $[0, \epsilon]$, and that $\int \beta(a) d a=\theta$. Integrating (6.2.1) along the characteristics (see section 4) gives

$$
n(t, a)=b(t-a) \exp \left(-\int_{0}^{a} \epsilon^{-1} \zeta(\alpha / \epsilon) p(t-a+\alpha) d \alpha=b(t-a) \exp \left(-\int_{0}^{a / \epsilon} \zeta(\tau) p(t-a+\epsilon \tau) d \tau\right),\right.
$$

which on taking formal limits for $\epsilon \downarrow 0$ reduces to

$$
n(t, a)=b(t-a) \exp (-\theta p(t-a))
$$

for all $a>0$. Taking the limit for $a \downarrow 0$ final, gives (6.2.3) again.

The first who tried to modify Gurtin's \& Levine's model by the incorporation of a functional response was Frauenthal (1983). Again no mechanistic route was taken. Following Diekmann et al (1986) (who concentrate on cannibalism) we shall now show how the previous derivation should be extended in this case.

We start assuming that

- The support of a function $f$ is defined as closure $\{x \mid f(x) \neq 0\}$ 


$$
\alpha(t, a)=\beta(a) p(t) \Phi(c),
$$

where $0 \leqslant \Phi(c) \leqslant 1$ is a reduction factor e.g. due to prey handling times and

$$
c(t)=\int_{0}^{\infty} h(a) \beta(a) n(a) d a,
$$

where $h$ is an age specific weight function e.g. describing how handling time depends on prey age (compare exercise I.2.2.3). The well known Holling disk equation gives $\Phi(c)=1 /(1+c)$ but other functions may be inserted. As before we find for $\epsilon \downarrow 0$ the recruitment rate

$$
n(0+)=b e^{-\theta p \Phi(c)} .
$$

However, the calculation of the limiting $c$ for $\epsilon \downarrow 0$ (remember that $\beta$ in $(6.2 .4 \mathrm{~b}$ ) was $\epsilon$-dependent) is a more subtle problem. From

$$
c(t)=\int_{0}^{1} h(\epsilon \sigma) \zeta(\sigma) b(t-\epsilon \sigma) e^{-\int_{\delta}^{\delta} \zeta(\tau) p(t-\epsilon \sigma-\epsilon \tau) \Phi(c(t-\epsilon \sigma-\epsilon \tau)) d \tau} d \sigma
$$

we obtain for $\epsilon \downarrow 0$

$$
c(t)=h(0) b(t) \int_{0}^{1} \zeta(\sigma) e^{-p(t) \Phi(c(t)) \int_{0}^{\infty} \zeta(\tau) d \tau} d \sigma=\frac{h(0) b(t)}{p(t) \Phi(c(t))}\left(1-e^{-\theta p(t) \Phi(c(t))}\right),
$$

which we can rewrite as

$$
\frac{c \Phi(c)}{h(0)}=\frac{b}{p}\left(1-e^{-\theta p \Phi(c)}\right)
$$

(6.2.5b) is a consistency relation: The left hand side equals the limit for $\epsilon \downarrow 0$ of $\int_{0}^{\infty} \beta(a) n(a) d a \Phi(c)$ which is the number of prey eaten per predator per unit of time (i.e. the functional response), and the right hand side equals the total number of prey eaten per unit of time divided by the number of predators.

The question remains whether for given $b$ and $p$ (6.2.5.b) defines a unique $c$. The biological interpretation requires the left hand side to be an increasing function of $c$ which is zero for $c=0$. If, as in Holling's disk equation, $\Phi$ is a decreasing function of $c$ with $\Phi(\infty)=0$ then a unique solution $c=c(b, p)$ exists (see figure 6.2.1) (It seems possible in principle that several solutions coexist if the functional response is $s$-shaped but we did not pursue this complication).

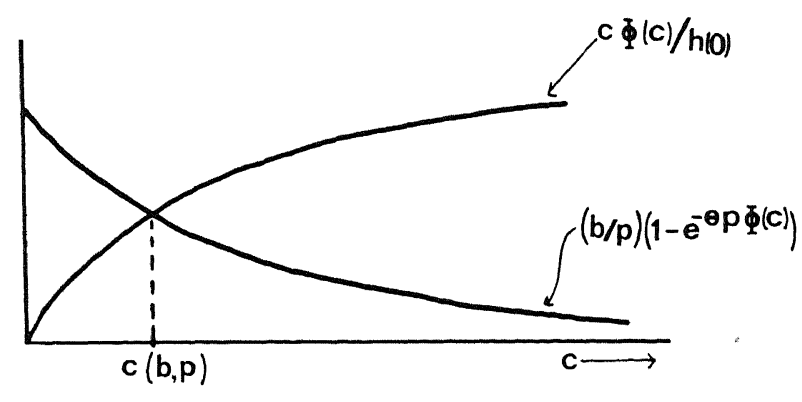

Fig. 6.2.1: The determination of the quantity $c(b, p)$.

In the case of cannibalism $p$ corresponds to a certain part of the prey population itself. Thus one might take

$$
p=\int_{0}^{\infty} k(a) n(a) d a,
$$

where $k$ is an age specific index of cannibalistic tendency. (See Diekmann et al (1985) for a detailed analysis of this situation.) If cannibalism acts indiscriminately among juveniles only, as e.g. in Tribolium (see Fujii (1978) and the references therein) then $\beta$ and $k$ have the same support and moreover are constant on this support (or, if we make the more general assumption that the juvenile period has a stochastic duration, $\beta$ and $k$ are both proportional to the probability that an individual of age $a$ is still in the juvenile stage). In that case in the limit for $\epsilon \downarrow 0, p$ equals some fixed constant times $c$. This special case is elaborated further in VI.3. Exercise 6.2 .8 discusses, in the context of the next 
example, how the model should be modified when the coincidence between the vulnerable and cannibalistic classes is less than exact.

EXAMPLE 6.2.4: Nursery competition. Technically this example is closely related to the previous one but the biological process is completely different. We consider a larval biotope which contains a limited number of "safe places" (e.g. spots to hide for predators or spots with abundant food). Larvae have to compete for these places. Those larvae which are temporarily excluded from the safe places have an increased risk of dying. Using a time scale argument we model this by letting the death rate depend on the fraction of the time that the individual is not in a safe place. The latter quantity of course depends on the number of competitors $m$. So instead of (6.2.2) we put

$$
\alpha(t, a)=\beta(a) F(m)
$$

and identify (compare the discussion at the end of the previous example)

$$
m=\int_{0}^{\infty} \beta(a) n(a) d a .
$$

Thus $\beta$ describes the age dependence of both the activity in the competition process and the need for a safe place (in principle these two could also be different, safe places being occupied by individuals who do not really need them), and $F$ describes how the death rate depends on the effective number of competitors $m$. (In the appendix to this subsection we shall derive an explicit expression for $F$ from a mechanistic submodel on a fast time scale.)

With $F$ at our disposal we may normalize $\beta$ to have integral one. Setting again $\beta(a)=\epsilon^{-1} \zeta(a / \epsilon)$ and letting $\epsilon \downarrow 0$ we arrive at

$$
\begin{aligned}
& n(0+)=b e^{-F(m)}, \\
& m=(b / F(m))\left(1-e^{-F(m)}\right) .
\end{aligned}
$$

The consistency relation (6.2.8b) again allows a simple interpretation. To this end we rewrite it as

$$
m F(m)=b\left(1-e^{-F(m)}\right) .
$$

Both the left and the right hand sides now equal the number of larvae dying per unit of time.

The biological interpretation requires that $F$ is an increasing function of $m$. This implies (see exercise 6.2 .6 below) that (6.2.8b) has a unique solution $m=m(b)$.

Combining (6.2.8) with the usual balance laws for births and deaths gives the population model

$$
\begin{array}{ll}
\frac{\partial n}{\partial t}+\frac{\partial n}{\partial a}=-\nu n, & n(0+)=b e^{-F(m)}, \\
b=\int_{0}^{\infty} B(a) n(a) d a, & m=(b / F(m))\left(1-e^{-F(m)}\right),
\end{array}
$$

where $B$ and $\nu$ are the age specific birth and death rates.

In IV.1.3 we shall reformulate this model as a nonlinear renewal equation in $b$ (see also exercise 6.2.7). In VI.3 this renewal equation will be analyzed for its dynamic properties.

EXERCISE 6.2.5: Derive (6.2.8b)

EXERCISE 6.2.6: Prove that for increasing $F(6.2 .8 \mathrm{~b})$ allows a unique solution.

Hint: First show that $f(x)=\left(1-e^{-x}\right) / x$ decreases for $x>0$, and then appeal to a graphical argument. .

EXERCISE 6.2.7: Convert (6.2.9) into an integral equation for $b$.

Hint: Introduce the function $h$ defined by $h(b)=b e^{-F(m)}, m$ the solution of $(6.2 .8 \mathrm{~b})$. The answer to this exercise can be found in IV example 1.3.1.

EXERCISE 6.2.8: An essential point in the argument in example 6.2.4 was that the vulnerability to competition and the involvement in competition depend in exactly the same manner on age, as expressed by the appearance of the same function $\beta$ in both (6.2.7a) and (6.2.7b). To see this set $\alpha(t, a)=\epsilon^{-1} \zeta(a / \epsilon) F(m)$ and $m=\epsilon^{-1} \int_{0}^{\infty} \eta(a / \epsilon) d a$. For $\epsilon \downarrow 0$ we get again $n(0+)=e^{-F(m)}$ in combination with an equation for $m$ of the form $m=b G(m)$. Find an expression for $G$. This expression will still contain a number of integrals. In order to arrive at an algebraic expression assume that $\zeta$ is a step function which equals 1 for $a<1$ and zero after, and that $\eta$ is the same sort of step function except that the step is made at $1+\delta, \delta>-1$. Calculate an explicit expression for $G$. 
EXAMPLe 6.2.9: The PPP-problem: the air plancton. Till now we have refrained from discussing the searching, patchless prey and predators. In the hints to the various exercises we have simply said that the overall densities of searching prey and predators were to be denoted as $P$ and $Q$ respectively.

$P$ and $Q$ have to be calculated from an equation for the "air plancton", to coin a phrase from Sabelis (these notes), i.e. those prey and predator individuals that have taken the wing after their patch got exhausted. It seems reasonable to describe these populations by an age dependent model, to describe the effects of exhaustion, desiccation etc. Assuming that exhaustion only influences death rates, but not searching efficiency, we may write

$$
\begin{aligned}
& P=\int_{0}^{\infty} p(a) d a, \quad \frac{\partial p}{\partial t}=-\frac{\partial p}{\partial a}-\mu p-\zeta p n_{0}, \quad p(0)=R, \\
& Q=\int_{0}^{\infty} q(a) d a, \quad \frac{\partial q}{\partial t}=-\frac{\partial q}{\partial a}-\nu q-q \int_{0}^{x_{m}} \eta(x) n_{1}(x) d x, \quad q(0)=S,
\end{aligned}
$$

(remember $n_{0}$ and $n_{1}$ were the densities of the empty and the prey patches respectively and $x$ was the within patch prey density), where $R$ and $S$ denote respectively the production of searching prey and predators due to patch exhaustion. $\zeta$ is the rate constant of empty patch encounter by searching prey and $\eta$ is the rate constant of prey patch encounter by searching predators.

When $\mu$ and $\zeta$ are both large (and continuous in their arguments) (6.2.10) can be simplified to (with some selfevident abuse of notation)

$$
\zeta P=\frac{\zeta R}{\mu(0)+\zeta n_{0}},
$$

and when $\nu$ and $\eta$ are both large (6.2.11) can be simplified to

$$
\eta Q=\frac{\eta S^{\prime}}{\nu(0)+\int_{1}^{x_{m}} \eta(\xi) n_{1}(\xi) d \xi} .
$$

(NB $\zeta P$ and $\eta Q$ are precisely the quantities that appear in the $p$-equations for the patches.)

EXERCISE 6.2.10: Write the time scale arguments by which (6.2.12) and (6.2.13) are derived out in full.

\subsection{A. An explicit expression for $F$ from the nursery competition model from example 6.2.4.}

During our derivation the number of competitors is assumed to be fixed. We assume that the total number of safe places is fixed at $s$. These places can be divided into occupied, $s_{1}$, and empty ones, $s_{0}$. The number of competitors temporarily deprived of a safe place equals $m-s_{1}$, (i.e. we assume that a safe place can be occupied by at most one individual). A law of mass action assumption now leads to

$$
\frac{d s_{1}}{d t}=-\lambda s_{1}+\mu s_{0}\left(m-s_{1}\right)=-\lambda s_{1}+\mu\left(s-s_{1}\right)\left(m-s_{1}\right)
$$

which has the stable equilibrium

$$
\hat{s}_{1}=\frac{m+s+(\lambda / \mu)-\sqrt{(m-s-\lambda / \mu)^{2}+4(\lambda / \mu) m}}{2}
$$

The number of competitors which has to do temporarily without a safe place is then given by

$$
H(m)=m-\hat{s}_{1}=\frac{1}{2}\left\{m-\xi+\sqrt{(m-\xi)^{2}+\rho m}\right\}
$$

where by definition

$$
\xi=s+\lambda / \mu, \quad \rho=4 \lambda / \mu \text {. }
$$

Finally, we observe that the fraction of the time that an arbitrary competitor is unsafe equals the probability that it is unsafe at any particular time, which equals $H(m) / m$. Therefore we put

$$
F(m)=\theta H(m) / m \text {, }
$$

where $\theta$ is the factor introduced by normalizing $\beta$ to have integral one.

In the following exercises some properties of $F$ are derived which will be needed in Chapter VI.3. 
EXERCISE 6.2A.1: Show that $H(m)=\frac{\rho}{4 \xi}\left\{m+\frac{1}{\xi}\left(1-\frac{\rho}{4 \xi}\right) m^{2}+O\left(m^{3}\right)\right\}$ for $m \downarrow 0$. Note that $(6.2 \mathrm{~A} .1 \mathrm{~b})$ implies that $1-\frac{\rho}{4 \xi}>0$ !

EXERCISE 6.2A.2: Show that $d H / d m=\frac{H(m)+\rho / 4}{\sqrt{(m-\xi)^{2}+\rho m}}>0$.

Hint: differentiate the quadratic equation for $H$.

EXERCISE 6.2A.3: Show that $d H / d m<1$ by first observing that $d H(0) / d m<1$ and then deriving a contradiction from the assumption $d H / d m=1$ for some $m$.

EXERCISE 6.2A.4: Show that $d^{2} H / d m^{2}=2 d H / d m \frac{1-d H / d m}{\sqrt{(m-\xi)^{2}+\rho m}}>0$.

EXERCISE 6.2A.5: Show that $\lim _{m \downarrow 0} \frac{m d H / d m-H(m)}{m^{2}}=\frac{1}{4} \frac{\rho}{\xi^{2}}\left(1-\frac{\rho}{4 \xi}\right)>0$.

EXERCISE 6.2A.6: Show that $m d H / d m-H>0$.

EXERCISE 6.2A.7: Show that $\frac{d F}{d m}=\theta \frac{m d H / d m-H}{m^{2}}>0$.

\subsection{Laws of large numbers on the individual level: the step from particulate to nonparticulate}

A second type of limit argument that often comes in useful is related to the law of large numbers from probability theory or the continuum approximations from physics. We have encountered this type of limit already in the first example from chapter $\mathrm{I}$, the invertebrate predator. There we introduced the assumption that the prey were very numerous as well as very small. The result was an equation in which the jump terms were replaced by an additional contribution to the continuous $i$-state movement, which we called the Rashevsky limit. Below we shall give one more example to illustrate the idea.

EXAMPLE 6.3.1: Size dependent reproduction in ectotherms, 2nd finale. When treating this model in chapter I we remarked that in our birth term

$$
b=\int_{0}^{l m} \lambda(l) n(l) d l, \quad g\left(l_{b}\right) n\left(l_{b}\right)=b,
$$

we made the implicit and very unbiological assumption that all individuals in the population contributed infinitesimal shares to a common pool from which the individual young are produced. Here we shall derive this, or rather a slightly modified, birth term from a limit argument, showing that at least (6.3.1) can be given a mechanistic underpinning. (Aldenberg's contribution in part B shows, in the context of biological production calculations, that such hairsplitting indeed has its uses!).

Since we wish to argue in energetic terms we shall in our arguments replace length $l$ by weight $w$. Now consider an animal that reproduces by forming buds of size $w_{0}$. The $p$-differential generator for such an animal may take the form

$$
\begin{aligned}
& \frac{\partial n}{\partial t}=-\frac{\partial g n}{\partial w}-\alpha n+\alpha\left(w+w_{0}\right) n\left(w+w_{0}\right)-\mu n, \\
& g\left(w_{0}\right) n\left(w_{0}\right)=\int_{w_{0}}^{w_{m}} \alpha(w) n(w) d w .
\end{aligned}
$$

We now derive an approximating $p$-equation by letting $w_{0}$ decrease to zero. As a first step we observe that for the same amount of energy spent one may make many small or one large young. Therefore we set $\alpha(w)=w_{0}^{-1} \beta(w)$. As a next step we consider the jump terms in the differential equation,

$$
-w_{0}^{-1} \beta(w) n(w)+w_{0}^{-1} \beta\left(w+w_{0}\right) n\left(w+w_{0}\right) .
$$

Taylor expanding the second term gives for $w_{0} \downarrow 0$ 


$$
-w_{0}^{-1} \beta(w) n(w)+w_{0}^{-1}\left(\beta(w) n(w)+\frac{\partial}{\partial w}\left(\beta(w) n(w) w_{0}+o\left(w_{0}\right)\right)=\frac{\partial}{\partial w} \beta(w) n(w)+o(1) .\right.
$$

Therefore in the limit for $w_{0} \downarrow 0$ the $p$-differential equation becomes

$$
\frac{\partial n}{\partial t}=-\frac{\partial}{\partial w}((g-\beta) n)-\mu n
$$

which corresponds to the $i$-differential equation

$$
\frac{d w}{d t}=g(w)-\beta(w)
$$

i.e. the energy chanelled to reproduction is subtracted from growth.

At the boundary we get into trouble. First we have to assume that individuals can grow away from zero. (Our discussion of the boundary $\partial_{0} \Omega$ for this example in 3.4 shows how this is naturally the case for Von Bertalanffy growth). But also the number of young born per unit of time explodes for $w_{0} \downarrow 0$. This can be repaired by the biologically reasonable assumption that very small young have a much larger chance of dying in the early stages of their life than large young have. To make things simple we shall assume that these deaths occur immediately after birth. The probability of "egg" survival we put equal to $\gamma w_{0}$. In that case for $w_{0} \downarrow 0$

$$
g(0) n(0)=\int \gamma \beta(w) n(w) d w .
$$

REMARKS 6.3.2: (i) (6.3.3b) differs from (6.3.1) in the location of the boundary, $l_{b}$ in (6.3.1), 0 in (6.3.3b). In practice this will not make a great difference if $g$ is relatively large on $\left(0, l_{b}\right)$ as is the case in the Von Bertalanffy growth equation used in our model formulation.

(ii) For many invertebrates the chanelling of energy to reproduction happens in a manner different from that assumed in (6.3.2): the weight of egg masses or their precursors cannot be lumped with individual weight. This means that we have to introduce egg mass as an additional $i$-state variable. In the spirit of our previous setup we may for example assume that this variable increases at a rate $\beta$, and that the animal reproduces as soon as the egg mass reaches a given value which equals the clutch size times the mass of one egg. However, there is a certain risk in such an approach. Once the course of the food density as a function of time is given, the course of the state of an individual is completely determined as well. This means that there is no spread in the size-egg mass plane among individuals in one cohort. So we get into the same kind of trouble as we sketched for the age-size dependent model from example 1.2. More important, however, is that the birth events of a cohort become completely predictable: the mean birth rate of a representative individual becomes a sequence of delta-functions. Example 6.1.1 shows that such a non-smooth production of offspring may entail a lack of convergence of the $p$-state to a stable $i$-state distribution. This is exactly the kind of effects we have to be on our guard for when analyzing structured population models. In our simplified Daphnia model from I.3 the smoothing action of the implicit randomness of the reproduction process kept any such trouble at bay.

EXERCISE 6.3.3: Instead of pulling an egg survival factor out of the hat we can introduce an age dependent death rate $\mu$ such that $\mu(a) \uparrow \infty$ for $a \downarrow 0$, which we rig in such a way that the increase in the production of young when we let $w_{0} \downarrow 0$, is precisely compensated by a decreasing survival through the initial age interval. What should this $\mu$ look like?

It is also possible to combine time scale arguments and "law of large number limits" in a more intricate manner than was done above. For example Metz \& van Batenburg (1985a) describe a limit for the invertebrate predator model of the form, for the individual,

$$
\frac{d s}{d t}=-a s+x g_{0}(s) \Phi\left(x g_{0}(s), s\right)
$$

where $s$ is again individual satiation and $\Phi$ is a handling time correction factor as in (6.2.4a). And this is but one more of the variety of possible limit arguments: we cannot describe them all, but we hope that you have got the taste from our examples.

\section{* 6A. A justification for the limit arguments: the Trotter-Kato Theorem}

In order to justify the limit transitions discussed in this and the previous section mathematically, we can sometimes use a so-called Trotter-Kato theorem, provided we are dealing with linear equations all the way. Such a theorem relates the convergence of differential generators (or, rather, resolvents; see chapter II) to the convergence of the associated semigroups. Here we shall state a version which seems useful in the context of linear structured population models. 
Let $X$ be a Banach space. Let for all $n \in \mathbb{N}$ the closed operator $A_{n}$ with (densely defined) domain $\mathscr{D}\left(A_{n}\right)$ generate a strongly continuous semigroup $T_{n}(t)$. Let $X_{0}$ be a closed subspace of $X$. Let $T(t)$ be a strongly continuous semigroup on $X_{0}$ with infinitesimal generator $A$. Finally let $D \subseteq \mathscr{D}(A)$ satisfy: for all $\psi \in \mathscr{D}(A)$ there exists a sequence $\psi_{n} \in D$ such that $\psi_{n} \rightarrow \psi$ for $n \rightarrow \infty$ (D is sometimes called a core for $\left.A\right)$.

THEOREM (Trotter-Kato). If for all $\psi \in D$, there is a $\psi_{n} \in \mathscr{Q}\left(A_{n}\right), n \in \mathbb{N}$, such that $\psi_{n} \rightarrow \psi, A_{n} \psi_{n} \rightarrow A \psi$ for $n \rightarrow \infty$, then for all $t_{0}>0$ and $\psi \in X_{0}$ one has

$$
\lim _{n \rightarrow \infty} \sup _{0 \leqslant t \leqslant t_{0}}\left\|T_{n}(t) \psi-T(t) \psi\right\|=0
$$

We refer to Davies (1980) and Pazy (1983a) for alternative versions of the Trotter-Kato theorem.

As an example of the application of this theorem we refer to Heijmans (1985), where the Trotter-Kato theorem is used to justify the Rashevsky limit from I.2.7.

\section{A. Calculus in $\mathbb{R}^{n}$, a short refresher}

\section{A1. Differentiation}

Let $f: X=\mathbb{R}^{n} \rightarrow Y=\mathbb{R}^{m}$ (or more generally let $X$ and $Y$ be Banach spaces, see II interlude 1.2). We call $f($ Fréchet) differentiable at $x_{0}$ if there exists a linear map denoted as $D f\left(x_{0}\right)$ such that

$$
\left\|f\left(x_{0}+h\right)-f\left(x_{0}\right)-D f\left(x_{0}\right) h\right\|=o(\|h\|) \text { for }\|h\| \downarrow 0,
$$

where $\|z\|:=\left(\sum z_{i}^{2}\right)^{\frac{1}{2}}$ is the Euclidian norm in $\mathbb{R}^{n}$ (or the norm of the Banach space of which $z$ is an element), and

$$
g(h)=o(\|h\|): \Leftrightarrow\|g(h)\| /\|h\| \downarrow 0 \text { for }\|h\| \downarrow 0 .
$$

The function $D f: X \rightarrow \mathfrak{L}(X, Y): x \mapsto D f(x)$ (where $\mathfrak{\varrho}(X, Y)$ denotes the space of continuous linear maps from $X$ to $Y$ ), if it exists, is called the derivative of $f$.

Some rules applying to $D f$ are $^{*}$

$$
D(g(f(x))=D g(f(x)) \cdot D f(x) \quad(\text { chain rule })
$$

$$
\text { If } Y=\prod_{i=1}^{k} Y_{i} \text { then } D f=\left(\begin{array}{c}
D f_{1} \\
\cdot \\
\cdot \\
D f_{k}
\end{array}\right) \quad \text { (componentwise differentiation) }
$$

(iii) If $X=\prod_{i=1}^{k} X_{i}$ then $D f(x) h=\sum_{i=1}^{k} D_{i} f(x) h_{i}=\left(D_{1} f(x), \cdots, D_{k} f(x)\right) h \quad$ (partial differentiation)

Applying (ii) and (iii) to the one dimensional components of $x$ and $y$ gives

$$
D f(x)=\left(\begin{array}{ccc}
\frac{\partial f_{1}}{\partial x_{1}} & \cdots & \frac{\partial f_{1}}{\partial x_{n}} \\
\cdot & & \cdot \\
\frac{\partial f_{m}}{\partial x_{1}} & \cdots & \frac{\partial f_{m}}{\partial x_{n}}
\end{array}\right)
$$

This matrix representation of $D f(x)$ is called the Jacobian matrix.

( $A$ 1.2) allows us to identify $D f$ with the map $D f: \mathbb{R}^{n} \rightarrow \mathbb{R}^{m \times n}$, and define the second derivative of $f$ as $D^{2} f:=D D f$, and so on. (When $X$ and $Y$ are more general Banach spaces $\mathfrak{L}(X, Y)$ is supplied with the usual operator norm, see interlude II.1.2, to arrive at the same result.) The notation $C(\Omega, Y)$ or $C^{0}(\Omega, Y), \Omega \subseteq X$, is used to denote the space of all bounded continuous functions $f: \Omega \rightarrow Y$ provided with the norm ${ }^{\dagger}$

"We shall think of $\mathbf{R}^{n}$ as a space of "column vectors". ${ }^{T}$ will denote transpose.

$\dagger$ The concept of norm in essence is a generalization of the concept of length of a vector. A formal definition may be 


$$
\|f\|_{0}:=\sup _{x \in \Omega}\|f(x)\|
$$

and more generally $C^{k}(\Omega, Y)$ is used to indicate the space of all $k$ times differentiable functions $f: \Omega \rightarrow Y$ with continuous derivatives up to the $k^{\prime}$ th, provided with the norm

$$
\|f\|_{k}:=\sum_{i=0}^{k} \sup _{x \in \mathbb{2}}\left\|D^{i} f(x)\right\|
$$

When $Y=\mathbb{R}$ one generally writes $C^{k}(\Omega)$ instead of $C^{k}(\Omega, Y)$. (The spaces $C^{k}(\Omega, Y)$ can all be shown to be Banach spaces.)

For $h \in X$ the directional derivative of $f$ in the direction of $h$ at $x_{0}$ is defined as

$$
\partial_{h} f\left(x_{0}\right)=\lim _{\epsilon \downarrow 0} \epsilon^{-1}\left(f\left(x_{0}+\epsilon h\right)-f\left(x_{0}\right)\right) .
$$

When $f$ is differentiable in $x_{0}$ we have by the chain rule

$$
\partial_{h} f\left(x_{0}\right)=D f\left(x_{0}\right) h .
$$

A class of functions of special interest to us, as the $p$-states belong to this class, are the functions $f: \mathbb{R}^{n} \rightarrow \mathbb{R}$. In $\mathbb{R}^{n}$ we define the usual inner product

$$
x \cdot y:=x^{T} y=\sum_{i=1}^{n} x_{i} y_{i} .
$$

So $\|x\|^{2}=x \cdot x$. When a function $u: \mathbb{R}^{n} \rightarrow \mathbb{R}$ is differentiable the transpose of its derivative $D u$ is called the gradient of $u$, and denoted as $\nabla u$. Thus the gradient is a column vector of partial derivatives

$$
\nabla u=\left(\begin{array}{c}
\frac{\partial u}{\partial x_{1}} \\
\cdot \\
\cdot \\
\frac{\partial u}{\partial x_{n}}
\end{array}\right]
$$

and $D u h=\nabla u \cdot h$ and also $\partial_{h} u=\nabla u \cdot h$ (provided $\nabla u$ is well defined).

A second class of functions of special interest as these comprise both the vector fields of $i$-state speeds in $\mathbb{R}^{n}$ as well as the reparametrizations of $i$-state space, are the functions $w: \mathbb{R}^{n} \rightarrow \mathbb{R}^{n}$. For such a function $w$ the trace of its Jacobian matrix $D w=\left[\frac{\partial w_{i}}{\partial x_{j}}\right]_{\substack{i=1, \ldots, n \\ j=1, ., n}}$ is called the divergence of $w$ and denoted as $\nabla \cdot w$. So

$$
\nabla \cdot w=\sum_{i=1}^{n} \frac{\partial w_{i}}{\partial x_{i}}
$$

(Note that (A1.8) represents the recipe by which we calculate $\nabla \cdot w$; the definition $\nabla \cdot w:=$ trace $(D w)$ is just convenient for analytical purposes.) The determinant of the Jacobian matrix is known as the Jacobian determinant, or Jacobian for short. It is also denoted as

$$
\frac{\partial\left(w_{1}, \cdots, w_{n}\right)}{\partial\left(x_{1}, \cdots, x_{n}\right)}:=\operatorname{det}(D w)
$$

(Note that the Jacobian matrix, the divergence and the Jacobian are all functions of $x$ !) The so-called inverse function theorem tells us that $w$ is invertible on a neighbourhood of $x$ whenever $\operatorname{det}(D w)(x) \neq 0$.

REMARKS A1: (i) In the often used Einstein convention one drops the summation sign, while it is understood that summation takes place over any repeated indices (like $i$ in the last two summations.)

(ii) Often one writes $\vec{x}$ for vectors and $\vec{w}$ for vector valued functions.

(iii) The so-called Laplacian, occurring in diffusion equations, is the divergence of the gradient

$$
\Delta u=\nabla \cdot \nabla u=\sum_{i=1}^{n} \frac{\partial^{2} u}{\partial x_{i}^{2}}
$$

found in interlude II.1.2. 


\section{A2. Integration}

For functions defined on $\mathbb{R}^{n}$ differentiation and integration are no longer inverse operations. Since integration of vector-valued functions can be carried out componentwise we concentrate on functions $f: \mathbb{R}^{n} \rightarrow \mathbb{R}$. The Riemann integral of a function over a smooth simply connected domain $\Omega_{0}$, denoted as

$$
\int_{\Omega_{0}} f(x) d x:=\int_{\Omega_{0}} f(x) \Pi d r:
$$

is defined in exactly the same manner as in the one dimensional case: cover $\Omega_{0}$ with closed boxes with nonoverlapping interiors with sides of length $\epsilon$ and let $f_{i}^{ \pm}$denote the maximum resp. minimum value of $f$ on the $i$ th box, then

$$
\int_{\Omega_{0}} f(x) d x:=\lim _{\epsilon \downarrow 0} \epsilon^{n} \Sigma f_{i}^{+}=\lim _{\epsilon \downarrow 0} \epsilon^{n} \Sigma f_{i},
$$

provided the two expressions at the right give the same value.

Riemann's construction does not apply to unbounded functions. However, when $f$ only grows beyond bounds for $x$ going to the boundary of $\Omega_{0}$ we can extend the Riemann integral by calculating $\int_{\Omega} f(x) d x$ for a sequence of sets $\Omega_{i}$ growing to $\Omega_{0}$ such that $f$ is bounded on each $\Omega_{i}$, and taking limits.

An alternative integral concept, due to Lebesgue, is essentially based on a partitioning of the range instead of the domain of $f$. It can be defined as, conditionally on the two expressions after the $:=$ sign giving the same value,

$$
\begin{aligned}
\int_{\Omega_{0}} f(x) d x:= & \lim _{\epsilon \downarrow 0} \sum_{i=-\infty}^{\infty} \epsilon i \lambda\left(\left\{x \in \Omega_{0} \mid \epsilon(i-1)<f(x) \leqslant \epsilon i\right\}\right) \\
& =\lim _{\epsilon \downarrow 0} \sum_{i=-\infty}^{\infty} \epsilon(i-1) \lambda\left(\left\{x \in \Omega_{0} \mid \epsilon(i-1) \leqslant f(x)<\epsilon i\right\}\right)
\end{aligned}
$$

where $\lambda(B)$ denotes the so-called Lebesgue measure ("volume") of the set $B$. The $d x$ under the integral sign now also is interpreted as referring to this measure. The Lebesgue integral is more appropriate for our purposes as it allows a larger class of functions, e.g. the unbounded ones, to be integrated (but at the price of having to define the function $\lambda$ for rather complicated sets). For piecewise continuous functions on bounded domains the two integrals coincide so that you can effectively think in terms of Riemann's construction if you find that more convenient.

The notation $L_{1}(\Omega), \Omega \subset \mathbb{R}^{n}$, is used to indicate the space of functions $f: \Omega \rightarrow \mathbb{R}$ such that

$$
\|f\|_{1}:=\int_{\Omega}|f(x)| d x<\infty,
$$

where the integral should be interpreted in the sense of Lebesgue, provided with $\|\cdot\|_{1}$ as a norm, with the understanding that any two functions $f_{1}$ and $f_{2}$ such that $\lambda\left(\left\{x \in \Omega \mid f_{1}(x) \neq f_{2}(x)\right\}\right)=0$ will be considered identical objects. (The main advantage of Lebesgue's definition of the integral is that with this definition $L_{1}(\Omega)$ can be shown to be a Banach space.)

Under fairly general conditions which need not be detailed here (when doing specific calculations we shall always confine ourselves, possibly implicitly, to such classes of functions that these conditions are certainly fulfilled) we have the following rule for evaluating integrals

$$
\int_{\Omega_{0}} f(x) d x=\int_{x_{n} \in B_{n}} \ldots \int_{x_{1} \in B_{1}\left(x_{2}, \ldots, x_{n}\right)} f(x) d x_{1} \cdots d x_{n} \quad \text { (componentwise integration) }
$$

where

$$
B_{i}\left(x_{i+1}, . ., x_{n}\right)=\left\{z_{i} \mid z \in \Omega_{0}, z_{i+1}=x_{i+1}, . ., z_{n}=x_{n}\right\} .
$$

Another rule which frequently comes in useful tells us how to deal with a change of variables. In the one-dimensional case the transformation $x=g(y)$ gives

$$
\int_{a}^{b} f(x) d x=\int_{g^{-1}(a)}^{g^{-1}(b)} f(g(y)) g^{\prime}(y) d y
$$

where we have to assume that $g^{\prime}(y):=D g(y) \neq 0$ for $a<g(y)<b$. This formula applies independent of the sign of $g^{\prime}(y)$ due to the information contained in the order of the integration boundaries $g^{-1}(b)$ and $g^{-1}(a)$. If we adhere to the notation of an integral over a domain $\Omega_{0}$ we have to replace $g^{\prime}(y)$ by its absolute value. This immediately generalizes to the $n$-dimensional case except that the role of $\left|g^{\prime}(y)\right|$ is played by $|\operatorname{det} D g(y)|$, i.e. the absolute value of the Jacobian of $g$. The rule then becomes

$$
\int_{\Omega_{0}} f(x) d x=\int_{g^{-1}\left(\Omega_{0}\right)} f(g(y))|\operatorname{det} D g(y)| d y
$$


for any invertible differentiable function $g$.

Apart from integrals over a domain $\Omega_{0}$ of $\mathbb{R}^{n}$ we also need integrals over lower dimensional surfaces $\Sigma$ in $\mathbb{R}^{n}$. An example of particular interest are subsets of the boundary $\partial \Omega$ of the $i$-state space. Usually these surfaces consist of a finite number of $m$-dimensional differentiable manifolds or "smooth surfaces", joined together by a finite number of edges, differentiable manifolds being defined as surfaces which locally can be represented as the image of some differentiable map $g: \mathbb{R}^{m} \rightarrow \mathbb{R}^{n}$ which on its range allows a differentiable inverse ${ }^{*}$. In that case we can use the connection with $\mathbb{R}^{m}$ to define a Lebesgue measure $d \sigma$ on $\Sigma$. Below we shall demonstrate the idea for $\Sigma$ one dimensional as this is the only case for which we do concrete calculations. In that case one also speaks of line integrals, otherwise of surface integrals. The calculations for general surface integrals are analogous except for some technical details.

Consider a l-dimensional curve $\Sigma$ in $\mathbb{R}^{n}$ which can be represented as $g: \mathbb{R} \rightarrow \mathbb{R}^{n}$ with $g$ differentiable and $D g(t) \neq(0, \ldots, 0)^{T}$ for those values of $t$ for which $g(t) \in \Sigma$. For $\Sigma$ we define

$$
\int_{\Sigma} f d \sigma=\int_{g^{-1}(\Sigma)} f(g(t))\|D g(t)\| d t
$$

where $\|\cdot\|$ is the Euclidean norm in $\mathbb{R}^{n}$. It is possible to show that this definition is independent of the choice of $g$. By varying $g$ it is only possible to move more slowly or quickly along $\Sigma$, but the factor $\|D g(t)\|$ makes that the points on $\Sigma$ get a proportionally smaller or larger "weight". (The technical details of defining general surface integrals center around the calculation of the analogue of this factor $\|D g\|$.)

EXERCISE A2.1: Prove the assertion above.

Hint: choose a function $\tilde{g}$ instead of $g$ which can be written as $\tilde{g}(t)=g(h(t))$ and use the chain rule and integration rule (ii). Observe then that the assumption on $D g$ implies that any function $\tilde{g}$ satisfying the same assumption can indeed be written as $\tilde{g}=g(h)$.

* EXERcISe A2.2: What would you expect to be the analogue of $\|D g(t)\|$ in the definition of a surface integral in $\mathbb{R}^{3}$ (Hint: first concentrate on linear maps so that $D g$ and $g$ coincide and use your intuitive idea of surface area). Generalize to $(n-1)$-surfaces in $\mathbb{R}^{n}$.

Let $\Omega_{0}$ be a domain in $\mathbb{R}^{n}$ with a smooth boundary $\partial \Omega_{0}$. Then we can define at every point of $\partial \Omega_{0}$ a tangent (hyper) plane. The outward normal vector to this tangent plane with (Euclidean) length 1 we shall always indicate as $\nu$.

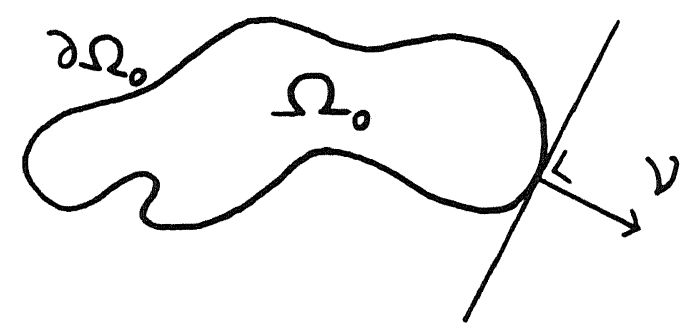

tangent plane

Fig. A.1

Using this notation the analogue of the $\mathbb{R}^{1}$ formula $\int_{a}^{b} f^{\prime}(x) d x=f(b)-f(a)$ for differentiable functions $u: \mathbb{R}^{n} \rightarrow \mathbb{R}$ is (iii) $\int_{\Omega_{0}} \nabla u d x=\int_{\partial \Omega_{0}} \nu u d \sigma$.

In this formula the requirement that $\partial \Omega_{0}$ is smooth can be relaxed to piecewise smoothness.

EXAMPLE A2.3: Let $\Omega_{0} \subset \mathbb{R}^{2}$ be defined by $\Omega_{0}=\left\{x \mid x_{1}^{2}+x_{2}^{2}<r\right\}$. We shall parametrize $2 \Omega_{0}$ with the angle $\phi$ as, $x_{1}=r \cos (\phi), x_{2}=r \sin (\phi)$. In other words $\partial \Omega_{0}$ is the image of the function $g:[0,2 \pi) \rightarrow \mathbb{R}^{2}: \phi \mapsto(r \cos (\phi), r \sin (\phi))^{T}$.

* With the notation $g: \mathbb{R}^{m} \rightarrow \mathbb{R}^{n}$ we do not necessarily mean that $g$ is defined on the whole of $\mathbb{R}^{m}$ as we are interested only in local properties. 


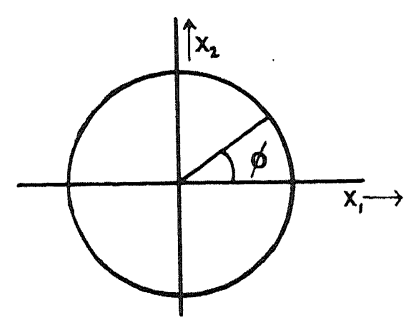

Fig. A.2

Then $D g(\phi)=(-r \sin (\phi), r \cos (\phi))$ and $\|D g(\phi)\|=r$. Now let $u(x)=x_{f}$ then $\nabla u(x)=(1,0)^{T}$ and

$$
\int_{\Omega_{0}} \nabla u(x) d x=\left[\begin{array}{c}
\pi r^{2} \\
0
\end{array}\right) .
$$

Since $\nu=(\cos (\phi), \sin (\phi))^{T}$ we have also

$$
\int_{\partial \Omega_{0}} \nu u d \sigma=\int_{0}^{2 \pi}\left[\begin{array}{l}
\cos (\phi) \\
\sin (\phi)
\end{array}\right] r \cos (\phi) r d \phi=r^{2} \int_{0}^{2 \pi}\left[\begin{array}{l}
\cos ^{2}(\phi) \\
\sin (\phi) \cos (\phi)
\end{array}\right) d \phi=\left[\begin{array}{c}
\pi r^{2} \\
0
\end{array}\right] .
$$

If we apply (iii) to each of the components of a function $w: \mathbb{R}^{n} \rightarrow \mathbb{R}^{n}$, and take the trace of the matrices on both sides of the equal sign we get

$$
\int_{\Omega_{0}} \nabla \cdot w(x) d x=\int_{\partial \Omega_{0}} \nu \cdot w d \sigma \quad \text { (divergence or Gauss' theorem). }
$$

(Note that this is a relation between the integrals of two scalar valued functions, one on the interior of $\Omega_{0}$ and one on $\partial \Omega_{0}$.) The importance of Gauss' theorem is that it tells us how to deal with mass balances in $\Omega_{0}$.

EXERCISE A2.4: Let $w: \mathbb{R}^{2} \rightarrow \mathbb{R}^{2}$ be defined by $w(x)=\left(x_{1}, x_{2}\right)^{T}$ and let $\Omega_{0}$ be as in the example above. Calculate both $\int_{\Omega_{0}} \nabla \cdot w(x) d x$ and $\int_{\partial \Omega_{0}} \nu \cdot w d \sigma$.

\section{A3. Some useful relations from linear algebra: the differentiation of determinants}

Let $M$ denote the square matrix

$$
M=\left[M_{i j}\right]_{j=1, \ldots n}^{i=1, ., n}=\left(m_{.1}, . ., m_{. n}\right)=\left(\begin{array}{c}
m_{1} . \\
\cdot \\
\cdot \\
m_{n}
\end{array}\right)
$$

where a dot instead of an index means that index is still left free, so $m_{1} .=\left(m_{11}, . ., m_{1 n}\right)$. The $i, j$ th minor $\left|M_{i j}\right|$ of $M$ is by definition the determinant of the $(n-1) \times(n-1)$ matrix obtained by deleting the $i$ th row and $j$ th column from $M$. The cofactor of the $i, j$ th component $m_{i j}$ of $M$ is defined as

$$
\text { cofactor } m_{i j}:=(-)^{i+j}\left|M_{i j}\right| \text {, }
$$

So the cofactor of $m_{i j}$ equals the determinant of the matrix obtained from $M$ by replacing the $j$ th column of $M$ by the $j$ th unit column vector (the $i$ th row of $M$ by the $i$ 'th unit row vector), and

$$
\operatorname{det} M=\sum_{j} m_{i j} \text { cofactor } m_{i j}=\sum_{i} m_{i j} \text { cofactor } m_{i j}
$$

Finally the adjoint of $M$ is defined as

$$
(\operatorname{adj} M)_{i j}:=\text { cofactor } m_{j i} \text {. }
$$


If $M$ is nonsingular Cramer's rule tells us that

$$
M^{-1}=(\operatorname{det} M)^{-1} \text { adj } M
$$

The map det: $\left(\mathbb{R}^{n}\right)^{n} \rightarrow \mathbb{R}:\left(m_{.1}, \ldots, m_{. n}\right) \rightarrow \operatorname{det} M$ is linear in its $n$ vectorial arguments $m_{. j}$. Therefore differentiating $\operatorname{det} M$ for $m_{. j}$ gives

$$
D_{m \cdot j} \operatorname{det}\left(m_{\cdot 1}, . ., m_{\cdot j}, . ., m_{\cdot n}\right) h=\operatorname{det}\left(m_{\cdot 1}, . ., h, . ., m_{\cdot n}\right)
$$

and from $\operatorname{det} M^{T}=\operatorname{det} M$

$$
h^{T} D_{m_{t}} \operatorname{det}\left(\begin{array}{c}
m_{1 .} \\
\cdot \\
\cdot \\
m_{i .} \\
\cdot \\
\cdot \\
m_{n .}
\end{array}\right)=\operatorname{det}\left(\begin{array}{c}
m_{1 .} \\
\cdot \\
\cdot \\
h^{T} \\
\cdot \\
\cdot \\
m_{n}
\end{array}\right) .
$$

Finally, by setting $h$ equal to the $i^{\text {th }}$ unit column vector in (A3.6) or direct from (A3.3)

$$
\frac{\partial \operatorname{det} M}{\partial m_{i j}}=\text { cofactor } m_{i j} \text {. }
$$

When our matrix $M$ is a function of a single variable $t$, by the chain rule

$$
\frac{d}{d t} \operatorname{det} M(t)=\sum_{i} \operatorname{det}\left(\begin{array}{c}
m_{1 .} \\
\cdot \\
\cdot \\
\frac{d m_{i .}}{d t} \\
\cdot \\
\cdot \\
m_{k .}
\end{array}\right)
$$

Now assume that

$$
\frac{d M}{d t}=B(t) M
$$

This relation can be rewritten as $\frac{d m_{i j}}{d t}=\sum_{k} b_{i k} m_{k j}$ or

$$
\frac{d m_{i}}{d t}=\sum_{k} b_{i k} m_{k},
$$

and therefore, by the chain rule

$$
\frac{d \operatorname{det} M}{d t}=\sum_{i} \sum_{k} b_{i k} \operatorname{det}\left(\begin{array}{l}
m_{1 .} \\
\cdot \\
m_{i-1}, \cdot \\
m_{k} \\
m_{i+1}, \cdot \\
\cdot \\
m_{n} .
\end{array}\right)=\sum_{i} b_{i i} \operatorname{det} M=\operatorname{trace} B \operatorname{det} M .
$$




\section{B. Stochastic continuous $i$-state movements}

So far we have excluded stochastic $i$-state movements from our considerations. The specification from first principles of $i$-models incorporating this type of movement requires a considerably greater insight in the detailed processes in and around the individual than we usually have, and we know of no model today in which a less than superficial connection with experimental data is made. Yet, as argued in remark 1.7, the incorporation of continuous stochastic $i$ state movements in our models may provide a description considerably closer to the actual biological mechanism than some of the ad hoc probabilistic postulates introduced earlier.

If we confirm ourselves to one-dimensional $i$-state spaces then, in the absence of jump contributions, the $p$ differential generators can be shown to be (see e.g. Cox \& Miller, 1965; Goel \& Richter-Dyn, 1979; Arnold, 1974; Karlin \& Taylor, 1981 or Wong \& Hajek, 1985)

$$
\frac{\partial n}{\partial t}=\delta_{t d} n+\delta_{t s} n=-\frac{\partial}{\partial x}(v n)+\frac{1}{2} \frac{\partial^{2}}{\partial x^{2}}\left(\sigma^{2} n\right)
$$

In this equation $v$ corresponds to the infinitesimal mean and $\sigma^{2}$ to the infinitesimal variance of the stochastic $x$ movement. The $i$-state $x$ itself follows a so-called diffusion process with drift $v$ and infinitesimal variance $\sigma^{2}$.

One possible way to derive expressions for $v$ and $\sigma^{2}$ is to start from a so-called stochastic differential equation for the $i$-state:

$$
\frac{d x}{d t}=u(x, t)+\sigma(x, t) w(t),
$$

where $w$ denotes an external stationary noise process with zero mean and

$$
\lim _{T \rightarrow \infty} T^{-1} \operatorname{var}\left(\int_{0}^{T} w(t) d t\right)=1
$$

EXAMPLE B.1: Stochastic cell growth. Let $x$ denote cell weight and let

$$
\frac{d x}{d t}=z a(x)-b(x),
$$

with $z$ the nutrient concentration. Let $z$ fluctuate around some mean value $y$ and let

$$
\alpha^{2}=\lim _{T \rightarrow \infty} T^{-1} \operatorname{var}\left(\int_{0}^{T} z(t) d t\right),
$$

then we can write

$$
\frac{d x}{d t}=[y a(x)-b(x)]+[\alpha a(x)] w .
$$

The next step is to assume that $w$ shows negligible memory, or, in other words, that $w$ approximates a so-called white noise. Then the $p$-equation becomes (Arnold, 1974; Karlin \& Taylor, 1981; Wong \& Hajek, 1985).

$$
\frac{\partial n}{\partial t}=-\frac{\partial}{\partial x}(u n)+\frac{1}{2} \frac{\partial}{\partial x}\left[\sigma^{2} \frac{\partial n}{\partial x}\right]=-\frac{\partial}{\partial x}\left(\left(u-\frac{1}{2} \frac{\partial \sigma^{2}}{\partial x}\right) n\right)+\frac{1}{2} \frac{\partial^{2}}{\partial x^{2}}\left(\sigma^{2} n\right)
$$

REMARKS B.2: (i) From (B.3) it appears that the noise also makes itself felt in the deterministic drift. However, this is partly a matter of convention. Clearly we cannot get away with the fact that the noise influences the infinitesimal mean. But we could have set

$$
\delta_{t d} n=-\frac{\partial}{\partial x}(u n) \text { and } \delta_{t s} n=\frac{1}{2} \frac{\partial}{\partial x}\left(\sigma^{2} \frac{\partial n}{\partial x}\right)
$$

instead of the arrangement suggested by (B.1). The term drift has a less strict connotion than infinitesimal mean. Therefore it might be possible to make some case to call $u$ from (B.3) the drift instead of $v$ from (B.1). Only when $\sigma^{2}$ is independent of $x$, and in particular when $\sigma^{2}=0$ (so that $\delta_{t s} n=0$ in either formalism), do we have coincidence of the two formalisms.

(ii) The assumption that $x$ qualifies as $i$-state implies that the $x$-process is not allowed to show any memory other than that inherent in the present value of $x$ itself. (This also was the reason that $w$ should be white noise.) A direct consequence is that it is impossible for the $x$-process to be differentiable as long as $\sigma^{2}(x)>0$. Therefore diffusion processes should at best be considered as approximations of considerably more complicated processes in which we leave out of 
the consideration all additional short memory components.

(iii) A direct consequence of the non-differentiability of the $x$-process is that for $w$ a white noise (B.2) cannot have its ordinary meaning. We have interpreted (B.2) here in the sense of Stratonovich (Arnold, 1974; Karlin \& Taylor, 1981; Wong \& Hajek, 1985). The reason is that we interpreted white noise as an approximation to a noise with a definite, even if short, memory. Only for the Stratonovich interpretation the map from $w$-process to $x$-process is continuous for $w$ going to a white noise. The Ito interpretation which is more commonly adhered to by mathematicians on account of its other good properties, fails in this respect.

(iv) In general a simple differential equation like (B.2) will itself be an approximation, based on a time scale argument, of a much more complicated underlying process. In writing down (B.2) we therefore implicitly assumed that the fast time scales of that underlying process were much faster than the time scale of the fluctuations of $w$. This may be tricky when the noise fluctuations are fairly fast as well, as we assumed later in our argument. If the time scales of the noise and of the fast processes underlying the $x$ process are of the same order of magnitude, we are forced to get the infinitesimal mean and variance of the limiting diffusion by a direct calculation, instead of by the two step strategy of first writing down (B.2) and then referring to the well-documented formula (B.3).

The $k$-dimensional analog of (B.1) reads

$$
\frac{\partial n}{\partial t}=\delta_{t d} n+\delta_{t s} n=\sum_{i} \frac{\partial}{\partial x_{i}}\left(v_{i} n\right)+\frac{1}{2} \sum_{i j} \frac{\partial^{2}}{\partial x_{i} \partial x_{j}}\left(c_{i j} n\right)
$$

where $C=\left[c_{i j}\right]_{j, j=1, . . k}$ denotes the, necessarily positive semi-definite, matrix of infinitesimal (co)variances. And the $k$-dimensional analog of (B.3) is

$$
\frac{\partial n}{\partial t}=-\nabla \cdot \phi, \quad \phi=\left(u-\frac{1}{2} C \nabla\right) n
$$

with

$$
u_{i}=v_{i}-\frac{1}{2} \sum_{j} \frac{\partial}{\partial x_{j}} c_{i j}
$$

$\phi$ again allows the interpretation of flux vector, just as in (3.1.8).

REMARK B.3: (B.4) and (B.5) show that our facile argument preceding (3.1.1) is considerably more tricky then it might seem. It certainly is possible to calculate the local mass change as the sum of the mass changes due to the fluxes in the direction of the various $i$-state variables. These fluxes themselves may be coupled, however, as is apparent from the occurrence of infinitesimal covariances.

In general (B.4) has to be complemented with side conditions. We shall only consider the nonsingular case and assume that $\operatorname{det} C$ is bounded away from zero and that $v$ is bounded. We shall again denote as $\partial_{-} \Omega$ that part of the boundary transgression of which is known from biological considerations to lead to immediate local loss. (Due to our assumption of nonsingularity there is no possibility of individuals being pushed over some part of the boundary as a consequence of the processes in the interior of $\Omega$ as in the, singular, deterministic case.) The boundary condition expressing such loss can be shown to be

$$
n(x)=0 \text { for } x \in \partial_{-} \Omega \text {. }
$$

The local rate of mass loss can be calculated in the usual manner from the flux vector $\phi$. In the same manner we shall again denote that part of the boundary where we know from biological considerations that there is influx of $p$-mass as $\partial_{+} \Omega$. Balancing the arrival rate $b_{0}$ per unit of surface area with the local flux then gives

$$
\nu(x) \cdot \phi(x)=b_{\partial}(x) \text { for } x \in \partial_{+} \Omega \text {. }
$$

Finally we shall denote as $\partial_{0} \Omega$ that part of the boundary on which we know that there is neither mass loss nor any influx, implying that

$$
\nu(x) \cdot \phi(x)=0 \text { for } x \in \partial_{0} \Omega \text {. }
$$

REMARK B.4: (B.4) allows a lot more room for singularities than only the case of a deterministic mass flow. A complete classification for the one-dimensional case may be found e.g. in Karlin \& Taylor (1981) or Goel \& Richter-Dyn (1979).

Continuous stochastic $i$-state movements have the property that they immediately smooth away any delta-functions 
present in the initial data. This also applies to delta-function valued source and sink terms. We only give the formulae for the one-dimensional case.

A sink term of the form $-\beta \delta(x-a) n(x)$ gives rise to the transition condition

$$
n(a+)=n(a-), \quad \phi(a-)-\phi(a+)=\beta n(a),
$$

and a source term of the form $c \delta(x-a)$ to

$$
n(a+)=n(a-), \quad \phi(a+)-\phi(a-)=c .
$$

(For mathematicians: In this case it is fairly easy to give a distributional interpretation to a $p$-equation containing a delta-function valued sink term. Such in contrast to the case $\delta_{t s} n=0$.)

EXAMPLE B.5: Deterministic binary fission combined with stochastic individual growth. The p-equation for this example is (remember $x_{1}$ was the value of $x$ at which the cells divided and crossing $x_{0}$ resulted in death)

$$
\begin{aligned}
& \frac{\partial n}{\partial t}=-\frac{\partial}{\partial x}(\nu n)+\frac{1}{2} \frac{\partial^{2}}{\partial x^{2}}\left(\sigma^{2} n\right) \\
& n\left(x_{1}\right)=n\left(x_{0}\right)=0 \\
& n\left(\frac{1}{2} x_{1}+\right)=n\left(\frac{1}{2} x_{1}-\right), \phi\left(\frac{1}{2} x_{1}+\right)-\phi\left(\frac{1}{2} x_{1}-\right)=2 \phi\left(x_{1}-\right)
\end{aligned}
$$

with

$$
\phi=v n-\frac{1}{2} \frac{\partial}{\partial x}\left(\sigma^{2} n\right)
$$

\section{The p-equations for the examples from section 1}

The invertebrate functional response

The $p$-equations for this example have already been given as (4.1.5). If there is a maximum gut capacity $\bar{s}<s_{m}$ then $\Omega$ has to be truncated at $\bar{s}$ and (4.1.5d) has to be replaced by

$$
-f_{0}(s) n_{0}(t, \bar{s})=\int_{0}^{\bar{\tau}} f_{1}(s) n_{1}(t, \bar{s}, \tau) d \tau,
$$

where

$$
\bar{\tau}=\tau_{e}-a^{-1} \log \left(\frac{a c-u}{\overline{a s}-u}\right) .
$$

\section{Size dependent reproduction in ectothermic animals}

The $p$-equation for this example has already been given as (4.3.2). In laboratory experiments where we feed with food that does not reproduce we may set

$$
\frac{d x}{d t}=\alpha-\beta x-\frac{\nu \xi x}{1+\xi x} p
$$

with

$$
p=\int_{0}^{a_{\max }} l^{2}(a) n(a) d a .
$$

A time scale argument based on the assumption that $\alpha, \beta, \nu>>1$ then gives

$$
x \approx \frac{\alpha \xi-\beta-\nu \xi p+\sqrt{(\alpha \xi-\beta-\nu \xi p)^{2}+4 \alpha \beta \xi}}{2 \beta \xi} .
$$

Reproduction by binary fission

The $p$--equation for this example is

$$
\frac{\partial n}{\partial t}=-\frac{\partial n}{\partial a}-\frac{\partial V n}{\partial x}-(\mu+b) n
$$




$$
n(0, x)=4 \int_{a_{0}}^{\infty} b(a, 2 x) n(a, 2 x) d a
$$

If division occurs as soon as the size passes a stochastic threshold having a density $g$, a survivor function $\mathcal{G}, \mathcal{G ( x )}=\int_{x}^{\infty} g(\xi) d \xi$, and a hazard rate $\gamma, \gamma=g / \mathcal{G}$, determined at birth, except that a cell needs to have passed the minimal age $a_{0}$ before it can divide, then the $p$-equation becomes

$$
\begin{aligned}
& \frac{\partial n}{\partial t}=-\frac{\partial n}{\partial a}-\frac{\partial V n}{\partial x}-(\mu+V \gamma) n \\
& n\left(a_{0}+, x\right)=G(x) n\left(a_{0}-, x\right) \\
& n(0, x)=4\left[(1-G(x)) n\left(a_{0}-, x\right)+\int_{a_{0}}^{\infty} V(2 x) \gamma(2 x) n(a, 2 x) d a\right]
\end{aligned}
$$

\section{Colony size distribution in the diatom Asterionella}

If we assume that bonds break exactly at age $A$ the $p$-equations become

$$
\begin{aligned}
& \frac{\partial n_{k}}{\partial t}=-\sum_{i=1}^{k} \frac{\partial n_{k}}{\partial a_{i}}-d\left(a_{1}\right) n_{k}+2 n_{k+1}\left(a_{1}, . ., a_{k}, A\right), \quad k=1,2, \cdots \\
& \frac{\partial n_{0}}{\partial t}=-\frac{\partial n_{0}}{\partial a_{0}}-d\left(a_{0}\right) n_{0} \\
& n_{k}\left(0, a_{2}, . ., a_{k}\right)=d\left(a_{2}\right) n_{k-1}\left(a_{2}, . ., a_{k}\right), \quad k=2,3, . . \\
& n_{1}(0)=\int_{A}^{\infty} d\left(a_{0}\right) n_{0}\left(a_{0}\right) d a_{0} \\
& n_{0}(A)=2 n_{1}(A)
\end{aligned}
$$

for $0<a_{1}<. .<a_{k}<A<a_{0}$, and $n_{k}$ the bond-age density of colonies of size $2^{k}$.

If bond breakage follows the rule from exercise (3.2.5), the $p$-equations are

$$
\begin{aligned}
& \frac{\partial n_{k}}{\partial t}=-\sum_{i=1}^{k} \frac{\partial n_{k}}{\partial a_{i}}-d\left(a_{1}\right) n_{k}-b\left(a_{k}\right) n_{k} \\
&+\sum_{j=k+1}^{\infty} 2^{j-k} \int_{a_{k}}^{\infty} \cdot \int_{a_{j-1}}^{\infty}\left[b\left(a_{k+1}\right)-b\left(a_{k}\right)\right] n_{j}\left(a_{1}, . . a_{j}\right) d a_{j} . d a_{k+1}, \quad k=1,2, \cdots \\
& \frac{\partial n_{0}}{\partial t}=-\frac{\partial n_{0}}{\partial a_{0}}-d\left(a_{0}\right) n_{0}+\sum_{j=1}^{\infty} 2^{j} \int_{a_{0}}^{\infty} . \int_{a_{j-1}}^{\infty} b\left(a_{0}\right) n_{j}\left(a_{0}, a_{2}, . ., a_{j}\right) d a_{j} . . d a_{2} \\
& n_{k}\left(0, a_{2}, . . a_{k}\right)=d\left(a_{2}\right) n_{k-1}\left(a_{2}, . ., a_{k}\right), \quad k=2,3, \ldots \\
& n_{1}(0)=\int_{0}^{\infty} d\left(a_{0}\right) n_{0}\left(a_{0}\right) d a_{0} \\
& n_{0}(0)=0 .
\end{aligned}
$$

The case of independently breaking bonds has already been treated in example 5.4.2.

\section{The PPP-problem}

Figure C.1 depicts $\Omega$. The upper right hand boundary of $\Omega$ is given by

$$
\frac{\delta}{\alpha}\left[(x-1)+\frac{\beta}{\gamma}(y-1)\right]=r_{0}
$$

The $p$-equations are

$$
\frac{\partial n_{0}}{\partial t}=-\zeta P n_{0}+B
$$




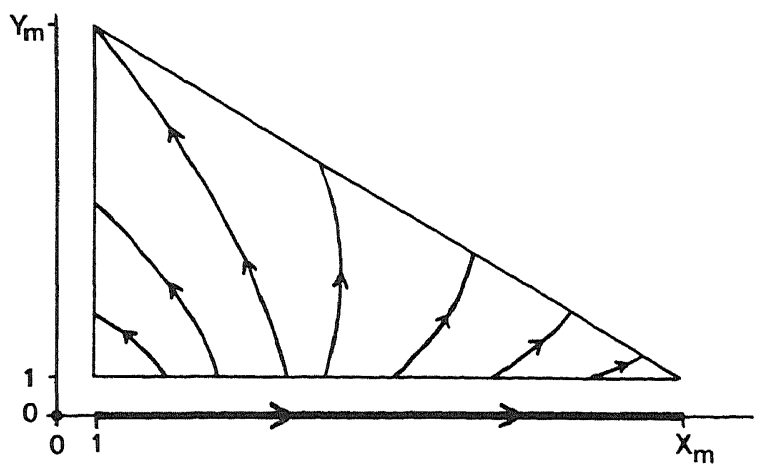

Fig. C.1. The $i$-state space of the PPP-problem

$$
\begin{aligned}
& \frac{\partial n_{1}}{\partial t}=-\frac{\partial \alpha x n_{1}}{\partial x}-\eta Q n_{1} \\
& \frac{\partial n_{2}}{\partial t}=-\frac{\partial(\alpha x-\beta y) n_{2}}{\partial x}-\frac{\partial \gamma y n_{2}}{\partial y} \\
& \alpha n_{1}(1)=\zeta P n_{0} \\
& \gamma n_{2}(x, 1)=\eta(x) Q n_{1}(x)
\end{aligned}
$$

The production rate of empty patches may be taken to be a function of the $p$-state as a whole. The simplest possibility is to set $B=f\left(n_{0}\right)$. $P$ and $Q$ have to be calculated from equations for the "air plancton". These have been discussed in example 6.2.9. The quantities $R$ and $S$ appearing there, corresponding to the production rates of "free" prey and predators respectively, can be calculated as

$$
\begin{aligned}
& R=\alpha x_{m}^{2} n_{1}\left(x_{m}\right)+\int_{1}^{x_{m}} \frac{\alpha \gamma}{\beta} x^{2} n_{2}\left(x, \frac{\gamma}{\beta}\left(\frac{\alpha}{\delta} r_{0}+1-x\right)+1\right) d x \\
& S=\int_{1}^{y_{m}} \alpha y n_{2}(1, y) d y+\int_{1}^{x_{m}} \frac{\alpha \gamma}{\beta} x\left(\frac{\gamma}{\beta}\left(\frac{\alpha}{\delta} r_{0}+1-x\right)+1\right) n_{2}\left(x, \frac{\gamma}{\delta}\left(\frac{\alpha}{\delta} r_{0}+1-x\right)+1\right) d x
\end{aligned}
$$

If $r_{0}$ is not a fixed number but a random variable with hazard rate $\kappa($ i.e. $\kappa(r)=-d \log \mathscr{K}(r) / d r$ where $\left.\mathcal{K}(r)=P\left(\left\{r_{0}>r\right\}\right)\right)$ then $(\mathrm{C} .9 \mathrm{~b})$ and $(\mathrm{C} .9 \mathrm{c})$ have to be replaced by

$$
\begin{aligned}
& \frac{\partial n_{1}}{\partial t}=-\frac{\partial \alpha x n_{1}}{\partial x}-\eta Q n_{1}-\delta x \kappa\left(\frac{\delta}{\alpha}(x-1)\right) n_{1} \\
& \frac{\partial n_{2}}{\partial t}=-\frac{\partial(\alpha x-\beta y) n_{2}}{\partial x}-\frac{\partial \gamma y n_{2}}{\partial y}-\delta x \kappa\left(\frac{\delta}{\alpha}\left((x-1)+\frac{\beta}{\gamma}(y-1)\right) n_{2}\right.
\end{aligned}
$$

and (C.10) by

$$
\begin{aligned}
& R=\int_{1}^{\infty} \delta x \kappa\left(\frac{\delta}{\alpha}(x-1)\right) n_{1}(x) d x+\int_{1}^{\infty} \int_{1}^{\infty} \delta x^{2} \kappa\left(\frac{\delta}{\alpha}\left((x-1)+\frac{\beta}{\gamma}(y-1)\right)\right) n_{2}(x, y) d x d y \\
& S=\int_{1}^{y_{m}} \alpha y n_{2}(1, y) d y+\int_{1}^{\infty} \int_{1}^{\infty} \delta x y \kappa\left(\frac{\delta}{\alpha}\left((x-1)+\frac{\beta}{\gamma}(y-1)\right)\right) n_{2}(x, y) d x d y
\end{aligned}
$$

Deterministic binary fission combined with stochastic individual growth

The p-equations for this example were already derived in example B.5, formula (B.11). 